\title{
Review Article \\ Potential Energy Surfaces Using Algebraic Methods Based on Unitary Groups
}

\author{
Renato Lemus \\ Instituto de Ciencias Nucleares, Universidad Nacional Autónoma de México, Apartado Postal 70-543, 04510 México, DF, Mexico \\ Correspondence should be addressed to Renato Lemus, renato@nucleares.unam.mx \\ Received 14 July 2011; Revised 13 October 2011; Accepted 21 October 2011 \\ Academic Editor: Sylvio Canuto \\ Copyright () 2011 Renato Lemus. This is an open access article distributed under the Creative Commons Attribution License, \\ which permits unrestricted use, distribution, and reproduction in any medium, provided the original work is properly cited. \\ This contribution reviews the recent advances to estimate the potential energy surfaces through algebraic methods based on the \\ unitary groups used to describe the molecular vibrational degrees of freedom. The basic idea is to introduce the unitary group \\ approach in the context of the traditional approach, where the Hamiltonian is expanded in terms of coordinates and momenta. \\ In the presentation of this paper, several representative molecular systems that permit to illustrate both the different algebraic \\ approaches as well as the usual problems encountered in the vibrational description in terms of internal coordinates are presented. \\ Methods based on coherent states are also discussed.
}

\section{Introduction}

The description of molecular systems involves the solution of the corresponding Schrödinger equation. This task is so difficult that an approach involving just numerical methods needs powerful computers even for three-or four-particle systems. An alternative approach is based on choosing the basis functions in such a way that they resemble the exact eigenfunctions as much as possible. The suitable basis are obtained by making approximations that simplify the Hamiltonian of the molecule. The advantage of this method is that the functions reproduce correctly the gross features of the spectrum, and consequently they provide a better physical insight in understanding the solutions. The first step in simplifying the molecular problem consists in taking advantage of the large difference between the nucleus and electron masses, a fact that leads to the Born-Oppenheimer approximation $[1,2]$. As a result of this approximation the original Schrödinger equation is split into two coupled equations, one corresponding to the electronic degrees of freedom which is solved for many nuclear geometries and the other one associated with the rotation-vibration Schrödinger equation for the nuclei whose potential is basically provided by the electronic energy $[3,4]$. On the other hand, the rotation-vibration Schrödinger equation is usually solved making the rigid-rotor approximation together with the harmonic oscillator approximation. The total wave function is then approximated as the direct product of three contributions: electronic, rotational, and vibrational wave functions. Corrections to this description are allowed by introducing the braking of the Born-Oppenheimer approximation, distortion effects, anharmonicity, centrifugal distortion, and Coriolis coupling [3-5].

Within the Born-Oppenheimer approximation the potential energy surface (PES) is provided by the solution of the electronic Schrödinger equation. Following this approach the calculation of a PES represents a major problem because of two main reasons. On one hand an accurate calculation of the electronic wave functions and energies is a quite difficult problem due to the correlation and exchange interactions, as well as the relativistic effects [6]. Hence, this problem is by itself a challenge of current interest. On the other hand the estimation of a PES involves the solution of the electronic Schrödinger equation for different nuclear configurations, a formidable computational task feasible only for small molecules. Even, when this situation is possible, the predicted vibrational spectrum generated from the obtained PES is far from the standards of high-resolution molecular vibrational spectroscopy, being necessary to refine the force constants in order to obtained a good vibrational description [7]. In summary, for medium and large molecules, the harmonic approximation is usually considered, with the proviso of a poor quality concerning the prediction of vibrational spectra. 
An alternative approach to obtain an estimation of the PES is provided by the vibrational spectrum of molecular systems $[3,4,8,9]$. An expansion of the kinetic energy and the potential in terms of normal coordinates, for instance, involves a set of force constants which can be estimated by fitting the vibrational spectrum. In this case the estimation of the PES is computationally cheap, with force constants fitted to provide a description of high quality. These force constants may in turn be used to predict spectra of isotopic species. The advantage of this approach is that it is relatively easy to implement, although the identification of the resonances and anharmonic interactions to establish the appropriate Hamiltonian to produce a high-quality fitting is in general not a simple task. The most common approach to carry out the vibrational description is through the use of the harmonic basis associated either with the normal modes or the symmetry-adapted coordinates. The inconvenience of taking a harmonic basis is that as the energy increases the admixture of states becomes evident due to the intrinsic properties of the basis. An alternative approach consists in considering a change to a more realistic basis. In particular a local basis turns out to be appropriate for two reasons. On one hand it allows the results provided by the normal basis to be recovered when local harmonic oscillators are considered, but on the other hand this selection opens the possibility of considering alternative bases, like Morse and/or Pöschl-Teller functions, which reflect more accurately the main physical properties of a pure local bond. Local bases emerged as a natural way to explain the spectra of molecules involving bonds with large differences in the masses of the begin and end atoms [10-13]. Although both alternatives, local or normal basis, may be worked out in configuration space, their corresponding algebraic representations provide an effective and elegant route to deal with the description of the vibrational degrees of freedom. When a harmonic basis is considered, the algebraic approach appears in natural form by introducing the bosonic operators associated with the creation and annihilation operators of the harmonic functions [14]. This is a relatively easy task that allows us to exploit the concept of polyad, a pseudo-quantum number that defines a subspace of states connected through the main interactions of the system [15]. In contrast, when considering anharmonic potentials, bosonic operators do not appear anymore, making the description a nontrivial task. Stimulated by this problem algebraic methods based on the unitary approach emerged as an alternative to describe the rovibrational degrees of freedom.

Unitary groups are proved to be relevant in the description of many-body systems. In fact bilinear products of creation and annihilation fermionic or bosonic operators form sets of generators of unitary groups, which constitute the dynamical group of a great variety of the systems [16]. In particular great attention has been paid to the description of electronic degrees of freedom in atoms and molecules [1720], nuclear physics [16, 21] and subnuclear physics [22], and also to the rovibrational degrees of freedom [23, 24]. When the vibrational excitations are described in terms of a basis of harmonic oscillators, unitary groups appear in natural form $[14,19,25]$. Bilinear products of creation and annihilation operators of a set of $v$ oscillators in $n$ dimensions constitute the generators of the symmetry group of the system $U(\nu n)$, while $\operatorname{sp}(2 \nu n, R)$ is the corresponding dynamical group $[14,26]$. A set of harmonic oscillators presents an infinite number of states, which explains the appearance of a noncompact group as a dynamical group. It is possible to work with compact dynamical groups if the space of harmonic oscillators is cut off. To achieve this goal an extra boson is added in such a way that the totally symmetric representation (total number of bosons) of the unitary group is fixed. This approach was for the first time proposed in the context of the description of collectives states of nuclei [27, 28]. Later on the same idea was applied by Iachello et al. in the field of molecular physics to describe the rotation-vibration excitations of molecular systems, establishing what is known as the vibron model $[23,29,30]$. In the framework of this approach a $U(4)$ group is associated with each bond providing a dynamical group in terms of the direct product of $U(4)$ groups. The vibron model was successful in describing linear molecules $[31,32]$, but, because of its increasing complexity, it does not become suitable for nonlinear molecules beyond triatomic systems. An alternative model to overcome this difficulty was proposed by considering the rovibrational degrees of freedom in unified form, proposing a unique unitary group $U(\nu+1)$ for a system of $v$ degrees of freedom including both vibrations and rotations [33]. In any case these models are phenomenological in the sense that the Hamiltonian is expanded in terms of the generators of the dynamical algebra (usually in terms of Casimir operators), providing eigenvectors and eigenvalues, but in purely algebraic form. In the framework of this formalism the PES may be extracted through the use of coherent states, an approach associated with the classical limit [23]. A relevant feature of the unitary approach is that the addition of the extra boson besides providing a compact group as a dynamical group, permits to take into account anharmonicities from the outset, enriching the model through the appearance of orthogonal subgroups, which play a preponderant role in the description of nonrigid molecules. In this type of systems, however, the polyad concept stops being useful. The full space has to be considered, allowing phase transitions to appear and giving rise to the possibility of describing molecular systems with several structural minima [3438].

Although most of the applications of the unitary group approach in the field of molecular spectroscopy have been developed to describe the rovibrational degrees of freedom, there exists a model for diatomic molecules including the full set degrees of freedom. In this model the $s p$ shell is considered for the electrons, while the rovibrational degrees of freedom are introduced through the $U(4)$ vibron model. The full Hamiltonian is then expanded in terms of the generators of the dynamical group $U^{e}(8) \times U^{r v}(4)$ [39-41]. A remarkable feature of this model is that the Born-Oppenheimer approximation is not assumed. The expectation values with respect to the coherent states allow the PES to be extracted for each electronic state. However, because the electronic degrees of freedom are taken in the 
united atom limit, the PESs do not reproduce the expected shape in the separated atoms limit $[42,43]$.

A unitary approach restricted to describe only vibrational excitations was developed by Michelot and Moret-bailly [44], which later on was further analyzed to include an additional subgroup in order to introduce the most important local interactions as a part of an expansion of the Hamiltonian in terms of Casimir operators [45]. This approach has been applied to several molecular systems, for instance, tetrahedral [46] and pyramidal molecules [47, 48], and it is based on the methodology of algebraic techniques where each chain of groups provides a dynamical symmetry that establishes a basis to diagonalize a general Hamiltonian, whose main contributions correspond to the Casimir operators associated with different chains [19]. In the framework of this approach for a set of $v$ equivalent oscillators, the dynamical group becomes $U(v+1)$. The relevance of this approach is not just the restriction of the space by itself but the fact that this treatment allows us to introduce anharmonicities from the outset. The one-dimensional version of this approach deserves special attention due to the fact that it is connected with the Morse and Pöshl-Teller potential, widely applied in molecular problems [19]. In this case the vibron model and the $U(v+1)$ approach coincide. This model was first identified to describe the stretching degrees of freedom, but later on it was extended to include the bending degrees of freedom $[49,50]$. As previously mentioned the extraction of the PES is a non-trivial task, although it is possible to estimate it through the coherent state formalism [23, $51,52]$. However, in order to be in position to calculate force constants useful to predict spectra of isotopic species, it is necessary to establish the appropriate correspondence between the coordinates and momenta of the system and the generators of the dynamical group [53].

In the last ten years successful efforts to connect the algebraic approaches to describe molecular systems based on unitary groups with their corresponding description in configuration space have been made. First the exact connection of the $U(2)$ model with the Morse and PöschlTeller potentials was established [54-56]. This connection allowed force constants to be obtained, and consequently the PES became available to predict spectra of isotopic species [57-62]. Later on a connection was established between the $U(v+1)$ model of $v$ equivalent oscillators proposed by Michelot and Moret-bailly [44] and the space of coordinates and momenta $[63,64]$. This connection allowed for the first time the calculation of PES in the framework of this algebraic model $[65,66]$. More recently, an explicit connection between the $U(3)$ algebraic model, used to describe the bending degrees of freedom of linear triatomic molecules [67], and the configuration space was established $[68,69]$.

The new approach developed to extract the PES may substitute the traditional approach in which the Casimir operators play a preponderant role, since it has the remarkable feature that every Hamiltonian written in terms of bosonic creation and annihilation operators associated with harmonic oscillators can be translated into the $U(\nu+1)$ approach in such a way that in the harmonic limit both treatments coincide. As a consequence, in this scheme it is not necessary to know the complex machinery of Lie algebras to apply the models. An additional feature of the algebraic approach is that it possesses particular features without analog in the configuration space regarding the descriptions where the polyad plays a fundamental role. It is always possible to obtain a better description of the vibrational excitations, compared with the analog models in configuration space at the same level of approximation. In addition an algebraic approach contrasts with the models based on $a b$ initio methods to extract the PES. The algebraic approaches are relatively simple to apply because the resonances can be established in a straightforward way and the matrix elements are calculated in a simple way. This feature allows a vibrational description to be done in a relatively short time compared with $a b$ initio methods or even with variational approaches where the kinetic energy is calculated in exact form and the potential is expanded to be fitted. However, it should be clear that the algebraic approaches cannot substitute the $a b$ initio calculations; they represent just an alternative to estimate the PES in a relatively simple and economic way when a molecular structure can be identified either for semirigid or nonrigid molecules, although in this paper we regard solely semirigid molecules. For instance, variational approaches are more reliable to make prediction out of the range of energies considered in the fits, and $a b$ initio calculations allow a potential energy surface of reactive molecular systems to be calculated. An algebraic approach is capable of providing PESs of two separate molecular systems, but it is not possible to obtain the effect of the molecular interaction over the PESs. It should be also mentioned that current computational procedures are capable of describing the rovibrational spectrum on a high level of approximation for molecules with four atoms like $\mathrm{NH}_{3}$, for instance [70]. Algebraic approaches, however, cannot contend with such calculations, they are proposed to establish approximated methods that may be applied to more complex systems where $a b$ initio calculations are too expensive to be applied.

In addition, in the context of molecular physics a potential energy surface may be obtained using an algebraic approach either by means of the connection of the spectroscopic parameters with the structure and force constants or by means of the introduction of the coherent states. However, only through the connection with configuration space it is possible to predict the spectra of isotopic species.

In this paper we review the recent advances in establishing the connection between the algebraic approaches based on the unitary groups and the physical space of coordinates and momenta. Our goal is to show the method to extract the PES for the different models and situations found in the description of vibrations in terms of internal coordinate. We start with the basic concepts involved in the algebraic approaches by analyzing the case of one oscillator. Later on the case of two oscillators is analyzed in detail to show the intrinsic advantages of an algebraic method. Here the approach using coherent states to extract a PES is also discussed. Thereafter several molecular systems are analyzed: nonlinear and linear triatomic molecules, pyramidal and planar molecules. This selection of molecules permits to 
illustrate the application of the different algebraic approach to estimate a PES.

This contribution is organized as follows. The basic concepts involved in the Born-Oppenheimer approximation is presented in Section 2. In Section 3 some fundamental concepts on symmetry are discussed. Section 4 is devoted to introduce the main ingredients of the algebraic approaches, while in Section 5 the connection of the $U(2)$ model with the Morse Potential is presented. In Section 6 we discuss in detail the case of two interacting Morse oscillators. An approach to extract the PES using coherent states is presented in Section 7. In Section 8 an approach to obtained the PES is illustrated presenting the study of the water molecule. In Section 9 a more elaborated system, the $\mathrm{BF}_{3}$, is presented. Section 10 is devoted to establish an approach to obtain the PES when the $U(v+1)$ algebraic models for nonlinear molecules are used. In Section 11 the case of linear molecules using the $U(3)$ model is analyzed. Finally in Section 12 the summary and conclusion are presented.

\section{Molecular Hamiltonian}

A molecule is a collection of $N$ nuclei and $\lambda-N$ electrons held together by forces and obeying the laws of quantum mechanics through the Schrödinger equation $\hat{H} \Psi=E \Psi$, where the Hamiltonian in the axis system at the molecular center of mass (parallel to the laboratory system) takes the general form $[3,4,71]$

$$
\hat{H}=\widehat{T}^{0}+\widehat{T}^{\prime}+\widehat{V},
$$

where $\widehat{T}^{0}$ is the sum of the kinetic energy of $\lambda-1$ particles (center of mass excluded), $\widehat{T}^{\prime}$ is a kinetic energy term that involves crossed terms among the $\lambda-1$ particles, while $V$ is the electrostatic potential energy. In our discussion interactions between electron-spin magnetic moments and between nuclear magnetic and electric moments are not considered. Although the molecular center of mass system allows the center of mass contribution to be eliminated, the problem of the cross-terms $\widehat{T}^{\prime}$ arises. To eliminate such cross-terms between nuclei and electrons, a reference system parallel to the laboratory at the nuclear center of mass is introduced. This new reference system induces in the kinetic energy the transformation $\widehat{T}^{0}+\widehat{T}^{\prime} \rightarrow \hat{T}_{e}+\hat{T}_{N}$, where $\widehat{T}_{e}$ and $\hat{T}_{N}$ are purely electronic and nuclear contributions. The electronic kinetic term $\widehat{T}_{e}$ contains diagonal $\hat{T}_{e}^{0}$ and crossed contributions $\widehat{T}_{e}^{\prime}$. If the latter is neglected, the Schrödinger equation takes the form

$$
\left\{\widehat{T}_{e}^{0}+\widehat{T}_{N}+\hat{V}\left(\mathbf{R}_{N}, \mathbf{r}_{e}\right)\right\} \Psi_{r v e}\left(\mathbf{R}_{N}, \mathbf{r}_{e}\right)=E_{r v e} \Psi_{r v e}\left(\mathbf{R}_{N}, \mathbf{r}_{e}\right),
$$

where $\mathbf{R}_{N}$ and $\mathbf{r}_{e}$ stand for the nuclear and electronic coordinates, respectively. Equations (2) is the starting point to carry out the Born-Oppenheimer (BO) approximation, which assumes that the motion of the electrons are unaffected by the motion of the nuclei. There are two ways of making the $\mathrm{BO}$ approximation: the perturbation theory approach
[1] and the variation theory approach [2]. In the former approach, the fundamental idea consists in expanding the rovibronic Hamiltonian in powers of the parameter $\kappa=$ $\left(m_{e} / M_{0}\right)^{1 / 4}$, where $m_{e}$ is the electron mass and $M_{0}$ is the mean nuclear mass. Identifying the different terms in powers of $\kappa$, the solutions are obtained in successive form. On the other hand, in the latter approach the rovibronic wave equation is written in the form

$$
\Psi_{r v e, m}\left(\mathbf{r}_{e}, \mathbf{U}_{N}\right)=\sum_{n^{\prime}} \Phi_{r v, n^{\prime}}^{m}\left(\mathbf{U}_{N}\right) \Phi_{\text {elec }, n^{\prime}}\left(\mathbf{r}_{e}, \mathbf{U}_{N}\right),
$$

where $\mathbf{U}_{N}$ are the vibrational displacement coordinates, $\Phi_{\text {elec }, n^{\prime}}\left(\mathbf{r}_{e}, \mathbf{U}_{N}\right)$ are a complete set of solutions to the electronic problem, and the coefficients $\Phi_{r v, n^{\prime}}^{m}\left(\mathbf{U}_{N}\right)$ are to be determined. Both treatments can be consulted in the literature $[1,2]$. Here we are going to provide only physical arguments for the zeroth-order solution.

The wave function in (2) may be factorized as a first approximation as the direct product of an electronic and a nuclear wave functions in the following form: $\Psi_{r v e}\left(\mathbf{R}_{N}, \mathbf{r}_{e}\right) \simeq \Phi_{e}\left(\mathbf{R}_{N} ; \mathbf{r}_{e}\right) \Phi_{r v}\left(\mathbf{R}_{N}\right)$, where the electronic function $\Phi_{e}\left(\mathbf{R}_{N} ; \mathbf{r}_{e}\right)$ is parametric in the nuclear positions and $\widehat{T}_{N} \Phi_{e}\left(\mathbf{R}_{N} ; \mathbf{r}_{e}\right) \Phi_{r v}\left(\mathbf{R}_{N}\right) \simeq \Phi_{e}\left(\mathbf{R}_{N} ; \mathbf{r}_{e}\right) \widehat{T}_{N} \Phi_{r v}\left(\mathbf{R}_{N}\right)$. Hence, freezing the nuclei in (2) and subtracting the nuclear repulsion term $V_{N N}$, the following equation for the electronic wave function is obtained:

$$
\left\{\widehat{T}_{e}^{0}+\hat{V}\left(\mathbf{R}_{N}, \mathbf{r}_{e}\right)-\widehat{V}_{N N}\right\} \Phi_{e}\left(\mathbf{r}_{e}\right)=V_{e}\left(\mathbf{R}_{N}\right) \Phi_{e}\left(\mathbf{r}_{e}\right) .
$$

If we now go back to (2) taking into account (4) and the previous considerations, we have for the nuclear equation

$$
\left\{\widehat{T}_{N}+\widehat{V}_{N}\right\} \Phi_{r v}\left(\mathbf{R}_{N}\right)=E_{r v} \Phi_{r v}\left(\mathbf{R}_{N}\right),
$$

where $\hat{V}_{N}=V_{e}+V_{N N}-E_{\text {elec }}$ and $E_{r v}=E_{r v e}-E_{\text {elec }}$, and the rotation-vibration energy for a bound electronic state is chosen so that the zero of energy is the minimum value of $V_{e}$. As a result of making the Born-Oppenheimer approximation, the original problem simplifies to the two differential equations (4) and (5), where the electronic equation has to be solved before (5) since the corresponding energy $V_{e}$ provides the potential in the nuclear equation.

Both the electronic and rotation-vibration equations are too complicated to be solved in exact form. The electronic equation belongs to the field of quantum chemistry [18, $71,72]$ and will not be discussed in this paper. We will concentrate our presentation on the calculation of the PES through the analysis of the vibrational degrees of freedom. We thus move to the analysis of the rotation-vibration equation, which is referred to as $(\xi, \eta, \zeta)$ axis system parallel to the laboratory with its origin at the nuclear center of mass. This equation can be separated into a rotational and a vibrational part by introducing a rotated $(x, y, z)$ system with its origin at the nuclear center of mass, also known as the molecule-fixed axis system. In matrix form we have

$$
\mathbf{r}_{\mathrm{mol}}=\hat{R}(\chi, \theta, \phi) \mathbf{r}_{\mathrm{lab}},
$$

where $R(\chi, \theta, \phi)$ is a rotation specified by the Euler angles [73]. In order to optimize the separation of the rotational 
and the vibrational parts, the Euler angles are determined through the Eckart equations [74]

$$
\sum_{i} m_{i} \mathbf{r}_{i}^{e} \times \mathbf{r}_{i}=0
$$

where $\mathbf{r}_{i}^{e}$ are the coordinates of the nuclear equilibrium configuration in the molecular-fixed system. For this configuration the orientation of the axis is chosen to correspond to the principal axis of inertia. When the nuclear coordinates are not far from the equilibrium, it turns out to be suitable to introduce the coordinates $\left\{\Delta \alpha_{i}=\alpha_{i}-\alpha_{i}^{e}, \alpha=x, y, z\right\}$ which are known as the vibrational displacement coordinates. In particular a linear combination of them corresponding to the normal coordinates $Q_{j}$ is appropriate to write down the molecular Hamiltonian. The rotation-vibration Hamiltonian involved in (5), when rewritten in terms of the molecular coordinates $\left\{\chi, \theta, \phi, Q_{i}, i=1, \ldots, 3 N-6\right\}$, for nonlinear molecules for instance, takes the form [75-77]

$$
\hat{H}_{r v}=\hat{H}_{\mathrm{rot}}^{0}+\hat{H}_{\mathrm{vib}}^{0}+V_{\mathrm{anh}}+\hat{V}_{\mathrm{cen}}+\widehat{V}_{\mathrm{cor}},
$$

where $\hat{H}_{\text {rot }}^{0}$ corresponds to the rotational contribution corresponding to the rigid-rotor Hamiltonian

$$
\hat{H}_{\mathrm{rot}}^{0}=\frac{1}{2} \sum_{\alpha} \mu_{\alpha \alpha}^{e} \hat{J}_{\alpha}^{2},
$$

the second contribution $\hat{H}_{\text {vib }}^{0}$ is a sum of independent harmonic oscillators associated with the normal modes

$$
\hat{H}_{\mathrm{vib}}^{0}=\frac{1}{2} \sum_{r}\left(\hat{P}_{r}^{2}+\lambda_{r} Q_{r}^{2}\right)
$$

while $V_{\text {anh }}$ contains the anharmonic contributions

$$
\widehat{V}_{\mathrm{anh}}=\frac{1}{6} \sum_{r, s, t} c_{r s t} Q_{r} Q_{s} Q_{t}+\frac{1}{24} \sum_{r, s, t, u} c_{r s t u} Q_{r} Q_{s} Q_{t} Q_{u}+\cdots,
$$

where the coefficients $c_{r s t . . .}$ are the force constants which determine the PES. The fourth term in (8) corresponds to the centrifugal distortion

$$
\hat{V}_{\text {cen }}=\frac{1}{2} \sum_{\alpha, \beta}\left(\mu_{\alpha \beta}-\mu_{\alpha \beta}^{e}\right)\left(\hat{J}_{\alpha}-\hat{p}_{\alpha}\right)\left(\hat{J}_{\beta}-\hat{p}_{\beta}\right),
$$

and the last term to the vibrational Coriolis coupling

$$
\hat{V}_{\text {cor }}=\frac{1}{2} \sum_{\alpha} \mu_{\alpha \alpha}^{e} \hat{p}_{\alpha}^{2}-\sum_{\alpha} \mu_{\alpha \alpha}^{e} \hat{J}_{\alpha} \hat{p}_{\alpha} .
$$

In these expressions $\hat{P}_{\alpha}$ is the conjugate momentum to the normal coordinate $Q_{\alpha}, \hat{J}_{\alpha}$ and $\hat{p}_{\alpha}$ are the components of the rotational and vibrational angular momenta, and $\mu_{\alpha \beta}$ is the inverse of the matrix $\mathbf{I}^{\prime}$ which involves the moments of inertia and the normal coordinates [75]. $\mu_{\alpha \beta}^{e}$ is the same matrix evaluated at equilibrium. Hence, the zeroth-order approximation corresponds to the rigid-rotor harmonic Hamiltonian $\hat{H}_{\mathrm{rv}}^{0}=\hat{H}_{\mathrm{rot}}^{0}+\hat{H}_{\mathrm{vib}}^{0}$, whose eigenfunctions provide suitable basis to diagonalize the complete Hamiltonian (8) for semirigid molecules.

Let us now focus on the vibrational degrees of freedom. Up to the seventies, the standard approach was based on the use of normal bases as stated by (10). The success of such a description at that time is explained because the analysis was restricted to the low lying region of the spectrum [8]. In the last decades, however, due to the development of new experimental techniques, it has been possible to obtain highresolution spectroscopic data for highly excited vibrational states $[78,79]$. In this region, however, it is rarely possible to find a dominant component to characterize the eigenstates, a feature due to the strong mixture of the harmonic basis. On the other hand, for high energies, the density of states increases rapidly, and, although the spectrum is expected to be more complex, in some cases some regularities appear, which can be explained in terms of a local oscillators scheme [11-13]. The pattern of the spectrum can be understood when one takes into account that in the chemical reactivity limit the energy tends to accumulate in some particular bonds where the reaction evolves. This behavior leads to consider the vibrational problem in terms of a set of interacting local oscillators.

The vibrational Hamiltonian $\hat{H}_{\text {vib }}$ in terms of curvilinear internal displacement coordinates $q_{i}$ acquires the general form $[80,81]$

$$
H=\frac{1}{2} \widetilde{\mathbf{p}} \mathbf{G}(\mathbf{q}) \mathbf{p}+V^{\prime}(\mathbf{q})+V(\mathbf{q}),
$$

where $\mathbf{q}$ and $\mathbf{p}$ are column vectors corresponding to the internal displacement coordinates and their conjugate momenta $\hat{p}_{k}=-i \hbar \partial / \partial q_{k}$, respectively, while the $\mathbf{G}(\mathbf{q})$ matrix establishes the connection between the internal and Cartesian coordinates. $V(\mathbf{q})$ is the Born-Oppenheimer potential, while $V^{\prime}(\mathbf{q})$ is a kinetic energy term not involving momentum operators which is usually neglected.

In a variational approach, the solution of the Schrödinger equation associated with the Hamiltonian (14) is obtained without any additional approximation. Since this approach is not viable for large- and even medium- sized molecules, approximate methods are welcome. The usual approach to obtain a suitable Hamiltonian to deal with consists in expanding both the $\mathbf{G}(\mathbf{q})$ matrix and the potential $V(\mathbf{q})$ as a Taylor series around the equilibrium configuration, truncating the expansion where an adequate convergence is achieved. In this scheme the zeroth-order Hamiltonian corresponds to a sum of harmonic local oscillators

$$
\hat{H}_{\mathrm{vib}}^{0}=\frac{1}{2} \sum_{\alpha} G^{e}(\mathbf{q}) \hat{p}_{\alpha}^{2}+\frac{1}{2} \sum_{\alpha} \lambda_{\alpha} q_{\alpha}^{2},
$$

where $\lambda_{\alpha}$ are the force constants. This Hamiltonian is diagonal in the basis of direct product of harmonic oscillator functions providing the basis to diagonalize a more general Hamiltonian. To obtain the zeroth-order Hamiltonian (15) from (14), it has been assumed an expansion in terms of the local coordinates $q_{i}$, a fact that leads to identify harmonic oscillators. A more suitable coordinates to carry out the expansion are Morse or Pöschl-Teller coordinates [60, 82], 
since in this way the zeroth-order Hamiltonian is identified with a set of Morse and/or PT oscillators. This provides from the outset a better description because of their extra degree of freedom of anharmonicity. An additional advantage is that both potentials can be treated in a unified form in terms of $s u(2)$ algebras, a crucial advantage that allows the vibrational description to be improved without analog in configuration space when the polyad is preserved. This point will be discussed later, but before going into details we briefly sketch some symmetry aspects of the molecular Hamiltonian.

\section{Symmetry Considerations}

The set of transformations that leaves the Hamiltonian invariant satisfies the properties of a group $G$. Technically speaking, if $R_{i} \in G$, then the associated operator $\hat{\mathcal{O}}_{R_{i}}$ acting on the physical space commutes with the Hamiltonian

$$
\left[\hat{\mathcal{O}}_{R_{i}}, \hat{H}\right]=0, \quad i=1,2, \ldots,|G|,
$$

where $|G|$ stands for the number of elements of the group. When the set of operations corresponds to the maximum number of transformation, $G$ is called the symmetry group of the system, and according to Wigner's theorem the eigenfunctions of the Hamiltonian span irreducible representations (irreps) of the group $G$ [83]. We thus have that the symmetry group depends on the Hamiltonian, and the action of the operators $\widehat{\hat{\mathcal{O}}}_{R_{i}}$ over the physical space should be specified in accordance with the approximation involved.

An exact Hamiltonian for any molecular system free of external fields is invariant under the following operations: (a) any translation along a space fixed direction, Euclidean group $E(3)$, (b) any rotation about a space fixed axis passing through the center of mass of the molecule, rotation group $S O(3),(c)$ any permutation of the space and spin coordinates of the electrons, permutation group $S_{n}^{(e)},(\mathrm{d})$ any permutation of the space and spin coordinates of identical nuclei, complete nuclear permutation group $G^{\mathrm{CNP}}$, and (e) inversion of the coordinates of all particles, nuclei and electrons, in the center of mass of the molecule, inversion group $\mathcal{E}$. These operations follow from the Hamiltonian and the nature of the space, uniformity, isotropy, indistinguishability of identical particles, and nature of the electromagnetic force. The full group is then given by

$$
E(3) \otimes S O(3) \otimes S_{n}^{(e)} \otimes G^{\mathrm{CNP}} \otimes \mathcal{E},
$$

where $G^{\mathrm{CNP}}$ is given in terms of the direct product of symmetric groups associated with identical nuclei. The group (17) provides irreps to label the states. For the Hamiltonian (1), where the kinetic energy of the center of mass has been eliminated, the Euclidean group is not included in (17). As noted this group does not contain the point group of the molecule. The reason is that the point group is associated with a specific structure of the molecule, which in fact is intrinsic to the Born-Oppenheimer approximation. Hence, there should be a connection between the true symmetries and point symmetries.
The group $G^{\mathrm{CNP}}$ depends only on the chemical formula, and it should be noted that its order can be very large. In practice systematic accidental degeneracies appear according to the label scheme provided by (17). These degeneracies are caused by the presence of more than one version of the equilibrium structure, in a given electronic state [3]. Different versions are connected through potential barriers which, when they are too high to be experimentally detected, the labeling scheme (17) provides extra labels manifested through degeneracy. From the point of view of symmetry, the operations of the complete permutation inversion group $G^{\mathrm{CNPI}} \equiv G^{\mathrm{CNP}} \otimes \mathcal{E}$ associated with the insuperable penetration of the barrier are said to be unfeasible, and a subgroup is more suitable to label the states. Hence, the group $G^{\text {CNPI }}$ may contain a subgroup composed of all feasible operations connecting several structural versions. This subgroup is known as molecular symmetry group (MS group). The MS group turns out to be isomorphic to the molecular point group when only one structural version is present. It should be stressed that the MS group depends on the resolution of the experiment, since the splitting of the inversion tunneling may or may not be detected, depending of the quality of the experimental devices.

From the technical point of view the distinction between different versions of the equilibrium structure is carried out by labeling the nuclei of the molecule in its equilibrium structure. By permuting the labels on identical nuclei with and without inverting the molecule, the number of versions can be identified, but it is only through the experiment that the MS group can be established. Permutation-inversion operations of the elements of MS group affect the coordinates of the nuclei and electrons in the molecule. It is through this effect that we are able to establish its connection with the molecular point groups. Each operation $p$ of the MS group can then be written in the following form [84-86]:

$$
p=\varphi(p) v(p) s(p)
$$

where $v(p)$ is an operation that produces the change in the vibronic coordinates (they translate into changes in the vibrational displacement coordinates as well as in the electronic coordinates) caused by $p, \varphi(p)$ is an operation changing the rotational coordinates (Euler angles), while $s(p)$ is an operation that generates a nuclear spin permutation. Since the operations $v(p), \varphi(p)$, and $s(p)$ affect different subspaces, they commute with each other. In semirigid molecules (where only one structural version is present) the vibronic operations $v(p)$ constitute the elements of the molecular point group isomorphic to the point group widely used when in the context of the Born-Oppenheimer approximation.

Let us now turn our attention to the identification of the irreps as a set of quantum numbers when discrete groups are involved. In essence, symmetry plays a central role in the necessity of establishing a complete set of commuting operators (CSCO) to label the eigenstates $|\Psi\rangle$ of the timeindependent Schrödinger equation. The Hamiltonian itself can be considered in the set of the CSCO, since the energy $E$ provides a label for the state. If $\alpha$ stands for an index 
introduced to distinguish different energies, the Schrödinger equation can be written as

$$
\hat{H}\left|\Psi_{i}^{\alpha}\right\rangle=E_{\alpha}\left|\Psi_{i}^{\alpha}\right\rangle, \quad i=1, \ldots, g_{\alpha},
$$

where $g_{\alpha}$ is the degeneracy. Because of the property (16), we may thus think that the set of operators $\left\{\hat{H}, \mathcal{O}_{R_{i}}, i=\right.$ $1, \ldots,|G|\}$ is useful to define a CSCO, but in general $\left[\mathcal{O}_{R_{i}}, \mathcal{O}_{R_{j}}\right] \neq 0$, unless the group is Abelian. This problem is solved by identifying subsets of $G$, which turn out to be the conjugate classes $K_{i}$ of the group, since $\left[\hat{K}_{j}, \hat{K}_{i}\right]=0$, for all $i, j$. Hence, the Hamiltonian together with the classes of the group constitute a set of commuting operators, whose representations can be diagonalized simultaneously in any space of independent functions.

We may now construct the representation matrix of the class $K_{p}$ in the basis of eigenvectors of the Hamiltonian: $\left\|\left\langle\Psi_{j}^{\alpha}\left|\widehat{K}_{p}\right| \Psi_{i}^{\alpha}\right\rangle\right\|$. The diagonalization of this matrix provides eigenvectors of type $\left\{\left|\Psi_{k}^{\alpha, \lambda_{p}}\right\rangle, k=1, \ldots, g_{\alpha, \lambda_{p}}\right\}$, with the property $\hat{H}\left|\Psi_{l}^{\alpha, \lambda_{p}}\right\rangle=E_{\alpha}\left|\Psi_{l}^{\alpha, \lambda_{p}}\right\rangle, \hat{K}_{p}\left|\Psi_{l}^{\alpha, \lambda_{p}}\right\rangle=\lambda_{p}\left|\Psi_{l}^{\alpha, \lambda_{p}}\right\rangle$, where $\lambda_{p}$ is the label that distinguishes the different eigenvalues of the class operator $\hat{K}_{p}$ and $l$ accounts for the degeneracy. We may now proceed to obtain the matrix representation of the next class $\hat{K}_{q}$ in the new basis $\left|\Psi_{l}^{\alpha, \lambda_{p}}\right\rangle$, to obtain eigenvectors labeled also with the eigenvalues $\lambda_{q}$. Following this approach for the rest of the classes leads to the states of the form $\left\{\Psi_{l}^{\left.\alpha, \lambda_{1}, \ldots, \lambda_{|K|}\right\}}\right.$ characterized by the eigenvalues $\left\{\alpha, \lambda_{1}, \ldots, \lambda_{|K|}\right\}$. However, this set of labels is not complete, since the number of irreps is equal to the number of classes, and consequently the set of labels $\left\{\lambda_{1}^{\nu}, \lambda_{2}^{v}, \ldots, \lambda_{|K|}^{\nu}\right\}$ specifies just the possible irreps $\nu$. The question which arises is concerned with the identification of the new set of operators capable to distinguish the states associated with the degeneracy of the irreps. The answer is given by the classes of a subgroup $H$, being $H \subset G$ a canonical chain. Suppose that $H$ has $|k|$ classes $\left\{k_{1}, \ldots, k_{|k|}\right\}$, which clearly satisfy $\left[k_{p}, k_{q}\right]=$ 0 . But the classes $K_{i}$ of the group $G$ commute with any element of the group and consequently commute also with the classes of the subgroup $\left[K_{i}, k_{p}\right]=0$, for all $i, p$. This fact suggests to diagonalize the representation of the operators $\hat{k}_{p}$ in the basis $\left\{\left|\Psi_{l}^{\alpha, \lambda_{1}, \ldots, \lambda_{|K|}}\right\rangle\right\}$ to obtain a complete labeling for the components of the irreps. After this procedure of diagonalization of the classes of $H$, we arrive to the complete labeling scheme

$$
\left|\Psi_{\lambda_{1}^{\mu}, \ldots, \lambda_{|k|}^{\mu}}^{\alpha, \lambda_{1 K \mid}^{\nu}, \ldots, \lambda_{|K|}^{\nu}}\right\rangle
$$

where the subindices $\lambda_{p}^{\mu}$ are defined by

$$
\hat{k}_{p}\left|\Psi_{\lambda_{1}^{\mu}, \ldots, \lambda_{|k|}^{\mu}}^{\alpha, \lambda_{1}^{\nu}, \ldots, \lambda_{|K|}^{\nu}}\right\rangle=\lambda_{p}^{\mu}\left|\Psi_{\lambda_{1}^{\mu}, \ldots, \lambda_{|k|}^{\mu}}^{\alpha, \lambda_{1}^{\nu}, \ldots, \lambda_{|K|}^{\nu}}\right\rangle
$$

Let us now turn our attention to the identification of the labels involved in (20) as quantum numbers. The time evolution of the expected value of an operator $\hat{A}$ is given by [87]

$$
\frac{d}{d t}\langle\Psi|\hat{A}| \Psi\rangle=\langle\Psi|[\hat{H}, \hat{A}]| \Psi\rangle+\left\langle\Psi\left|\frac{\partial \hat{A}}{\partial t}\right| \Psi\right\rangle,
$$

where $[\hat{H}, \hat{A}]$ is the commutator of the Hamiltonian with the operator $\hat{A}$. Hence, if the operator $\hat{A}$ does not depend explicitly on time and commute with the Hamiltonian, then the expected value is constant in time.

Suppose now that the states are chosen to be eigenstates of the Hamiltonian together with the classes of the group $G$ and subgroup $H$ :

$$
|\Psi\rangle \longrightarrow\left|\Psi_{\lambda_{1}^{\mu}, \ldots, \lambda_{|k|}^{\mu}}^{\alpha, \lambda_{1}^{\nu}, \ldots, \lambda_{|K|}^{\nu}}\right\rangle,
$$

then (22) translates into

$$
\begin{array}{r}
\frac{d}{d t} E_{\alpha}=0, \quad \frac{d}{d t} \lambda_{i}^{v}=0, \quad \frac{d}{d t} \lambda_{p}^{\mu}=0, \\
i=1, \ldots,|K|, \quad p=1, \ldots,|k|,
\end{array}
$$

when $\hat{A}$ is substituted by $\hat{H}, \hat{K}_{i}$, and $\hat{k}_{p}$. Hence, the eigenvalues of the set of operators $\left\{\hat{H} ; \hat{K}_{1}, \ldots, K_{|K|} ; \hat{k}_{1}, \ldots, k_{|k|}\right\}$ are independent of time and consequently are quantum numbers.

For a given $\alpha$ there are $|K|$ sets of $\lambda_{i}^{v}$ values. As mentioned before, this fact suggests a connection between the $\lambda_{i}^{v}$ values and the characters $\chi_{i}^{(\nu)}$ of the group. Indeed it has been proved the following connection [88]:

$$
\lambda_{i}^{v}=\frac{\left|K_{i}\right|}{n_{v}} \chi_{i}^{(v) *}, \quad i=1, \ldots,|K|,
$$

where $v$ stands for the irreps of the group, while $n_{v}$ refers to its dimension. A similar relation is also valid for $\lambda_{p}^{\mu}$ and the characters of the subgroup $H$.

The expression (25) provides a projection method. This assertion may be appreciated because of the following result: any symmetry-adapted function $\left|\psi_{i}^{(\nu)}\right\rangle$ spanning the $v$ th irreps of dimension $n_{v}$ satisfies [88]

$$
\hat{K}_{i}\left|\psi_{i}^{(\nu)}\right\rangle=\lambda_{i}^{v}\left|\psi_{i}^{(\nu)}\right\rangle, \quad i=1, \ldots, n_{\nu}
$$

which means that the functions $\left|\psi_{i}^{(\nu)}\right\rangle$ are eigenvectors of the class operators with eigenvalue $\lambda_{i}^{v}$. This remarkable result suggests to follow the inverse procedure to obtain (23). We can start diagonalizing the class operators to end up with the Hamiltonian. Consider this idea starting with an arbitrary set of functions $\left\{\left|\phi_{i}\right\rangle, i=1, \ldots, n\right\}$. First we chose a subset of classes that allows the irreps to be distinguished. The simultaneous diagonalization of the selected classes provides eigenvectors carrying the $v$ th irreps. The eigenvectors spanning the same irreps are then used to diagonalize the set of classes of the subgroup $H$. The resulting eigenvectors span irreps of the group $G$ as well as the subgroup $H$, allowing the components of degenerate irreps to be distinguished. This approach, proposed by Chen [88], turns out to be very powerful and has been used to generate general codes to project vibrational [89] and rotation-vibration functions [90].

\section{Basic Concepts of an Algebraic Approach}

The simplest model to describe the vibrational excitations of a molecule consists in modeling the system as set of 
interacting harmonic oscillators, in accordance with (10) and (11)

$$
\begin{aligned}
\hat{H}_{\mathrm{vib}}= & \frac{1}{2} \sum_{r}\left(\hat{P}_{r}^{2}+\lambda_{r} Q_{r}^{2}\right)+\frac{1}{6} \sum_{r, s, t} c_{r s t} Q_{r} Q_{s} Q_{t} \\
& +\frac{1}{24} \sum_{r, s, t, u} c_{r s t u} Q_{r} Q_{s} Q_{t} Q_{u}+\cdots
\end{aligned}
$$

An algebraic realization of this Hamiltonian can be obtained by introducing the operators [3]

$$
\begin{gathered}
a_{r}^{\dagger}=\frac{1}{\sqrt{2}}\left(\hat{P}_{r}+i \lambda_{r}^{1 / 2} Q_{r}\right), \quad \hat{a}_{r}=(1 \mid \sqrt{2})\left(\hat{P}_{r}-i \lambda_{r}^{1 / 2} Q_{r}\right), \\
{\left[\hat{a}_{r}, \hat{a}_{r}^{\dagger}\right]=\lambda_{r}^{1 / 2} \hbar,}
\end{gathered}
$$

which have the effect of ladder operators over the harmonic wave functions. This approach is known as the traditional algebraic approach for vibrational excitations [91]. The advantages of this description are that all matrix elements are calculated in algebraic form and that the resonances can be expressed in precise mode. As a consequence, it is easy to identify the interactions that preserve the polyad [15]. The harmonic basis intrinsic to this description, however, presents the disadvantage of an infinite dimension of the basis, a consequence that the potential does not reflect the appropriate behavior in the high energy region of the spectra. To overcome this problem, new algebraic approaches based on unitary groups emerged to take into account anharmonicities from the outset.

A fundamental concept intrinsic to the algebraic models is the dynamical group. The generators of this group form a Lie algebra, and any dynamical variable, including the Hamiltonian, can be expanded in terms of them. In this way the generators of the dynamical group possess the fundamental property that any pair of eigenstates of the Hamiltonian can be connected by them. Bilinear products of creation and annihilation operators of a set of $\nu$ harmonic oscillators in $n$ dimensions constitute the generators of the dynamical group $S P(2 v n, R)[14,26,92]$, a noncompact group presenting an infinite number of representations, in accordance with the infinite number of levels that present the harmonic oscillators. It is possible to work out with a compact dynamical group by adding an extra boson in such a way that the totally symmetric representation (total number of bosons) of the unitary group is fixed, as previously explained in the introduction. This ingredient simplifies enormously the description since the technical machinery involved in the compact groups is much less complicated than the one involved for the noncompact groups. To illustrate this approach, we will start considering the case of one oscillator.

Let us consider a one-dimensional harmonic oscillator, whose Hamiltonian and eigenvectors $|n\rangle$ in the algebraic representation take the form

$$
\hat{H}=\frac{\hbar \omega}{2}\left(\hat{t}^{\dagger} \hat{t}+\hat{t}^{\dagger}\right), \quad|n\rangle=\frac{1}{\sqrt{n !}}\left(\hat{t}^{\dagger}\right)^{n}|0\rangle .
$$

The symmetry group of this system is $U(1)$ since its generator $\hat{n}=\hat{t}^{\dagger} \hat{t}$ commutes with (29) [19]. On the other hand, the dynamical group is the noncompact group $S P(2, R)$. The unitary group approach consists in adding an extra boson $\widehat{s}$ in such a way that the total number of bosons $\hat{N}=\hat{t}^{+} \hat{t}+\widehat{s}^{+} \hat{s} \equiv$ $\hat{n}+\hat{n}_{s}$ is constant. The bilinear products $\left\{\hat{t}^{\dagger} \hat{t}, \hat{s}^{\dagger} \hat{s}, \hat{t}^{\dagger} \hat{s}, \hat{s}^{\dagger} \hat{t}\right\}$ are now generators of the $S U(2)$ group. The states associated with this group according to the chain

$$
U(2) \supset U(1)
$$

are given by [19]

$$
|[N], n\rangle=\frac{1}{\sqrt{(N-n) ! n !}}\left(\hat{s}^{\dagger}\right)^{N-n}\left(\hat{t}^{\dagger}\right)^{n}|0\rangle, \quad n=0,1, \ldots, N,
$$

where the generator of $U(1)$ is $\hat{n}=\hat{t}^{+} \hat{t}$. Because of the bosonic nature of the vibrations, these states span the totally symmetric representation $[N]$ of the $U(2)$ group. A connection of the generators of $U(2)$ with the known angular momentum generators in the Cartesian basis is given through [19]

$$
\begin{gathered}
\hat{J_{x}}=\frac{1}{2}\left(\hat{t}^{\dagger} \hat{s}+\hat{s}^{\dagger} t\right), \quad \hat{J_{y}}=\frac{1}{2 i}\left(\hat{t}^{\dagger} \hat{s}-\hat{s}^{\dagger} t\right), \\
\hat{J_{z}}=\frac{1}{2}\left(t^{\dagger} t-s^{\dagger} s\right)
\end{gathered}
$$

and in the spherical basis

$$
\begin{gathered}
\hat{J}_{+}=\hat{t}^{\dagger} \hat{s}, \quad \hat{J}_{-}=\hat{s}^{\dagger} \hat{t}, \\
\hat{J}_{0}=\frac{1}{2}\left(t^{\dagger} t-s^{\dagger} s\right) .
\end{gathered}
$$

Let us now consider the realization (33) with the following normalization:

$$
\begin{gathered}
\hat{c}^{\dagger} \equiv \frac{\hat{J}_{+}}{\sqrt{k}}=\frac{\hat{t}^{\dagger} \hat{s}}{\sqrt{k}}, \quad \hat{c} \equiv \frac{\hat{J}_{-}}{\sqrt{k}}=\frac{\hat{s}^{\dagger} \hat{t}}{\sqrt{k}}, \\
\hat{c}_{0} \equiv \frac{2 \hat{J}_{0}}{k}=\frac{1}{k}\left(t^{\dagger} t-s^{\dagger} s\right),
\end{gathered}
$$

where $k=N+1$. The action of these operators over the $U(1)$ kets (31) is

$$
\begin{gathered}
\hat{c}^{\dagger}|[N], n\rangle=\sqrt{(n+1)(1-(n+1) / k)}|[N], n+1\rangle, \\
\hat{c}|[N], n\rangle=\sqrt{n(1-n / k)}|[N], n-1\rangle .
\end{gathered}
$$

Note that the operators $\hat{c}^{\dagger}(\hat{c})$ connect the whole space of the functions $|[N], n\rangle$, and consequently $U(2)$ is the dynamical group of the system; any dynamical variable can be expressed in terms of the generators of the unitary group $S U(2)$. However, it is clear from (35) that it is only in the harmonic limit $N \rightarrow \infty$ for finite $n$ that the matrix elements of the onedimensional harmonic oscillator is recovered, for example, $\lim _{N \rightarrow \infty} \hat{c}^{\dagger}=t^{\dagger}$. Because of the constraint over $N$, a more general Hamiltonian is expected to be expanded in terms of the generators of the dynamical group $U(2)$ in such a way that $[\hat{H}, \hat{N}]=0$. The total number of bosons is related 
to $j$, the angular momentum label, through $j=N / 2$ [19]. In general it is convenient to establish the different chains of groups and the invariant operators associated with them since they are used to expand the Hamiltonian. In our case we have three more chains in addition to (30) that provide alternative bases

$$
\begin{aligned}
& U(2) \supset S O_{x}(2), \\
& U(2) \supset S O_{y}(2), \\
& U(2) \supset S O_{z}(2),
\end{aligned}
$$

where the subindex in the $S O(2)$ groups refers to the operator in (32) that generates the group. Hence, a possible simple expansion of the Hamiltonian is

$$
\hat{H}_{x}=A \widehat{J}_{x}^{2} .
$$

This Hamiltonian is diagonal in the $O_{x}(2)$ basis. If the basis associated with this chain is denoted by $\mid[N]$, $\mu\rangle ; \hat{J_{x}}|[N], \mu\rangle=\mu|[N], \mu\rangle$ and if in addition the following quantum number is defined $v=j+\mu$, with $v=0,1, \ldots, j-1$ when only the branch with negative values of $\mu$ is considered, the eigenvalues of $\hat{H}_{x}$ take the form

$$
\widehat{E}_{x}(v)=A \hat{\mu}^{2}=A j^{2}-2 A j\left(v-\frac{1}{2 j} v^{2}\right) .
$$

This is a Morse-like spectrum, which explains the fact that the $S_{x}(2)$ chain is associated with the Morse functions [19]. Analytical solutions obtained from Hamiltonians involving invariant operators associated with a chain are called dynamical symmetries. In general, the Hamiltonian that best suits the description involves invariant operators of several chains, and any basis can be used to diagonalize it. Hence, the introduction of the $s$-boson not only deforms the potential, but also enriches the description providing dynamical symmetries and alternative bases to carry out the calculations.

\section{Connection of the $U(2)$ Algebraic Model with the Morse Potential}

The Hamiltonian for one-dimensional Morse potential has the form [93]

$$
\hat{H}=\frac{1}{2 \mu} \hat{p}^{2}+D\left(1-e^{-\beta q}\right)^{2}
$$

where $\mu$ is the reduced mass, $q=x-x_{0}$ is the displacement coordinate, $D$ is the depth of the potential, and $\beta$ stands for its range. The solution of the Schrödinger equation associated with this Hamiltonian is given by

$$
\Psi_{v}^{j}(z)=N_{v}^{j} e^{-z / 2} z^{s} L_{v}^{2 s}(z)
$$

where $L_{v}^{2 s}(z)$ are the associated Laguerre functions, the argument $z$ is related to the physical displacement coordinate $q$ by $z=(2 j+1) e^{-\beta q}, N_{v}^{j}$ is the normalization constant and the variables $j$ and $s$ are related to the depth of the potential and the energy, respectively through [19]

$$
\kappa=2 j+1=\sqrt{\frac{8 \mu D}{\beta^{2} \hbar^{2}}}, \quad s=\sqrt{\frac{-2 \mu E}{\beta^{2} \hbar^{2}}},
$$

with the constraint condition $s=j-v$. Using the factorization method, it is possible to obtain creation $\hat{b}^{\dagger}$ and annihilation $\hat{b}$ operators of the Morse functions, which turn out to have the following effect:

$$
\begin{aligned}
\hat{b}^{\dagger} \Psi_{v}^{j}(z) & =\sqrt{(v+1)\left(1-\frac{v+1}{\kappa}\right)} \Psi_{v+1}^{j}(z), \\
\hat{b} \Psi_{v}^{j}(z) & =\sqrt{v\left(1-\frac{v}{\kappa}\right)} \Psi_{v-1}^{j}(z),
\end{aligned}
$$

with

$$
\hat{v} \Psi_{v}^{j}(z)=v \Psi_{v}^{j}(z)
$$

and whose explicit form in terms of the coordinate and momentum are given in [94]. The operators $\left\{b^{\dagger}, b\right\}$, together with the number operator $\hat{v}$, satisfy the commutation relations

$$
\left[\hat{b}, \hat{b}^{\dagger}\right]=1-\frac{2 \hat{v}+1}{\kappa}, \quad\left[\hat{v}, \hat{b}^{\dagger}\right]=\hat{b}^{\dagger}, \quad[\hat{v}, \hat{b}]=-\hat{b},
$$

which can be identified with the usual $s u(2)$ commutation relations through $\left\{b^{\dagger}=\hat{J_{-}} / \sqrt{\kappa}, b=\hat{J}_{+} / \sqrt{\kappa}, \hat{v}=j-\hat{J}_{0}\right\}$, where $J_{\mu}$ satisfy the usual "angular momentum" commutation relations [73]. Hence, the $S U(2)$ group is the dynamical group for the bound states of the Morse potential [19]. From the group theoretical point of view, the parameter $j$ labels the irreducible representations of the $S U(2)$ group. The projection of the angular momentum $m$ is related to $v$ by $m=j-v$. From this relation we see that the ground state $(v=0)$ corresponds to $m=j$, while from the dissociation condition the maximum number of quanta is $v=j(m=0)$. The state corresponding to $v=j$, however, is not normalizable, and consequently the allowed values for $v$ are $v=0,1, \ldots, j-1$. The Morse functions are then associated with one branch (in this case to $m \geq 1$ ) of the $S U$ (2) representations, although a recent work associates a noncompact group to the bound Morse space [95]. The bound solutions (40), however, do not form a complete set of states in the Hilbert space [96]. However, when the vibrational excitations are far from the dissociation limit, it is a reasonable approximation to consider the bound states as a complete space $[97,98]$. A similar situation appears in the case of the Pöschl-Teller potential $[96,99]$.

The realization of the Morse Hamiltonian in terms of the $s u(2)$ algebra is given by [58]

$$
\hat{H}^{M}=\frac{\hbar \omega}{2}\left(\hat{b}^{\dagger} \hat{b}+\hat{b} \hat{b}^{\dagger}+\frac{1}{2 \kappa}\right) .
$$

From (42) we obtain the corresponding eigenvalues

$$
E_{M}(v)=\hbar \omega\left[\left(v+\frac{1}{2}\right)-\frac{1}{\kappa}\left(v+\frac{1}{2}\right)^{2}\right] .
$$


The harmonic limit is obtained by taking $\kappa \rightarrow \infty$ :

$$
\lim _{\kappa \rightarrow \infty} \hat{b}=\hat{a}, \quad \lim _{\kappa \rightarrow \infty} \hat{b}^{\dagger}=\hat{a}^{\dagger},
$$

where the operators $\left\{\hat{a}^{\dagger}, \hat{a}\right\}$ satisfy the usual bosonic commutation relations. Since the set of operators $\left\{\hat{b}^{\dagger}, \hat{b}, \hat{v}\right\}$ constitutes a dynamical algebra for the Morse potential, any dynamical variable can be expanded in terms of them. In particular we are interested in the expansion of the momenta and Morse coordinates. For the momentum the following second-order expansion is obtained [55]:

$\hat{p}=\frac{i}{2} \sqrt{2 \hbar \omega \mu}\left\{f_{v} \hat{b}^{\dagger}-\hat{b} f_{v}+\frac{1}{\sqrt{\kappa}}\left(g_{v} \hat{b}^{\dagger} \hat{b}^{\dagger}-\hat{b} \hat{b} g_{v}\right)+O\left(\frac{1}{\kappa}\right)\right\}$,

while for the Morse coordinate [100, 101]

$$
\begin{aligned}
\frac{y}{\beta}= & \sqrt{\frac{\hbar}{2 \omega \mu}} \\
& \times\left\{f_{v} \hat{b}^{\dagger}+\hat{b} f_{v}+\frac{1}{\sqrt{\kappa}}\left(f_{v}^{d}+g_{v} \hat{b}^{\dagger} \hat{b}^{\dagger}+\hat{b} \hat{b} g_{v}\right)+O\left(\frac{1}{\kappa}\right)\right\},
\end{aligned}
$$

where $f_{v}, g_{v}$, and $f_{v}^{d}$ are functions of the number operator $\hat{v}$ given by

$$
\begin{gathered}
f_{v}=\sqrt{\frac{(\kappa-2 v-1)(\kappa-2 v+1)}{(\kappa-v)^{2}}}, \\
g_{v}=-\sqrt{\frac{\kappa^{2}(\kappa-2 v-1)(\kappa-2 v+3)}{(\kappa-v)^{2}(\kappa-v+1)^{2}}}, \\
f_{v}^{d}=1+2 v .
\end{gathered}
$$

Since $\hat{v}$ is diagonal, we have substituted $\hat{v}$ by $v$. For high number of quanta $v$ or $\kappa$ small, the terms of order $1 / \sqrt{\kappa}$ must be included. In contrast, if we are interested in the low lying region of the spectrum, a reasonable approximation consists in neglecting the terms of order $1 / \sqrt{\kappa}$ and taking the harmonic limit of the diagonal functions $\lim _{\kappa \rightarrow \infty} f_{v}=1$, $\lim _{\kappa \rightarrow \infty} g_{v}=-1$. We may thus propose the linear approximation

$$
\begin{gathered}
p \simeq \frac{i}{2} \sqrt{2 \hbar \omega \mu}\left(\hat{b}^{\dagger}-\hat{b}\right), \\
\frac{y}{\beta}=q \simeq \sqrt{\frac{\hbar}{2 \omega \mu}}\left(\hat{b}^{\dagger}+\hat{b}\right) .
\end{gathered}
$$

We immediately note the similarity of these expressions with the harmonic oscillator case. In fact, taking the harmonic limit we recover the usual expressions for the harmonic case. In this approximation it is clear that effective interactions preserving the polyad can be established in a straightforward way.

Let us now come back to the previous analysis of the algebraic $S U(2)$ model. If we compare the matrix elements (35) with (42), we immediately identify the isomorphism

$$
\hat{c}^{\dagger} \approx \hat{b}^{\dagger}, \quad \hat{c} \approx \hat{b}, \quad|[N], n\rangle \approx|j, v\rangle .
$$

However, we still have to identify the dynamical symmetry associated with the Morse Hamiltonian. The isomorphism (52) suggests the $\mathrm{SO}_{z}(2)$ symmetry since the eigenfunctions of $\hat{J}_{z}$ are also eigenfunctions of $\hat{n}$. In fact, if $\sigma$ is the eigenvalue of $\hat{J}_{z}$, we have the identity $\sigma=n-j$. Taking the negative branch of $\sigma$, we have $n=0,1, \ldots, j-1$. The $S O_{z}(2)$ dynamical symmetry takes the form

$$
\hat{H}=\frac{\hbar \omega}{2}\left(\hat{c}^{\dagger} \hat{c}+\widehat{c}^{\dagger}+\frac{1}{2 k}\right)
$$

which up to a constant is basically $\hat{J}_{z}^{2}$, as expected. We have thus established the exact connection between the $s u(2)$ algebraic model and the eigenfunctions of the onedimensional Morse Hamiltonian.

The connection we have presented deserves an additional comment. First we note that, in the linear approximation (51), the generator $\hat{J_{x}}$ is proportional to the Morse coordinate $y / \beta$, while $\hat{J_{y}}$ corresponds to the momentum. The dynamical symmetries $S_{x}(2)$ and $S_{y}(2)$ are then associated with the coordinate and momentum representation in the linear approximation. On the other hand, the $\mathrm{SO}_{z}(2)$ dynamical symmetry is associated with the energy representation, while the $U(1)$ corresponds to the energy representation in the harmonic limit.

The treatment presented here may be followed for the Pöschl-Teller potential arriving to a similar result; $S U(2)$ is the dynamical group of the system [56]. The matrix elements (35) are isomorphic to the corresponding matrix element of the creation and annihilation operator of the PT functions. Of course, the explicit form of the wave function is different as well as the expansion of the coordinate and momenta, but the energy spectrum is the same, as established by the $s u(2)$ algebra. There is a fundamental difference between these potentials, Morse and PT. While the Morse potential is asymmetric, the PT potential is symmetric, allowing both symmetric and asymmetric local modes to be modeled in the unified framework of the $s u(2)$ algebra.

\section{Two Interacting Morse Oscillators}

When the masses of the atoms involved in a set of equivalent bonds are very different, a scheme of interacting local oscillators represents a suitable approach to carry out the vibrational description. For example, the stretching modes in $\mathrm{H}_{2} \mathrm{O}$ present a strong local behavior because of the large ratio $16: 1$ of the masses. In contrast, when the masses are similar, the behavior is normal and starting from a set of local oscillators does not represent a good zerothorder approximation. It is possible, however, to describe molecules presenting a strong normal behavior in terms of local oscillators, even taking into account the preservation of the polyad, a fact that provides unique advantage of the algebraic models over the traditional description in configuration space, as we next explain.

We start our discussion with a treatment in terms of local harmonic oscillators. The Hamiltonian (14) for two 
equivalent oscillators up to quadratic order and neglecting $V^{\prime}(\mathbf{q})$ takes the form

$$
\hat{H}=\frac{1}{2} g_{r r}^{o} \sum_{i=1}^{2} \hat{p}_{i}+\frac{1}{2} f_{r r} \sum_{i=1}^{2} \hat{q}_{i}^{2}+g_{r r^{\prime}}^{o} \hat{p}_{1} \hat{p}_{2}+f_{r r^{\prime}} q_{1} q_{2} .
$$

Introducing the bosonic operators

$$
\hat{p}_{i}=\frac{i}{2} \sqrt{2 \hbar \omega \mu}\left(\hat{a}_{i}^{\dagger}-\hat{a}_{i}\right) \quad q_{i}=\sqrt{\frac{\hbar}{2 \omega \mu}}\left(\hat{a}_{i}^{\dagger}+\hat{a}_{i}\right) \quad i=1,2,
$$

with $\mu=1 / g_{r r}^{o}$ and $\omega=\sqrt{f_{r r} g_{r r}^{o}}$, the Hamiltonian (54) takes the form

$$
\begin{aligned}
\lim _{N \rightarrow \infty} \hat{H}= & \frac{\hbar}{2} \sqrt{f_{r r} g_{r r}^{o}} \sum_{i=1}^{2}\left(a_{i}^{\dagger} a_{i}+a_{i} a_{i}^{\dagger}\right) \\
& +\frac{\hbar}{2} \sqrt{f_{r r} g_{r r}^{o}}\left(\frac{f_{r r^{\prime}}}{f_{r r}}+\frac{g_{r r^{\prime}}^{o}}{g_{r r}^{o}}\right)\left(a_{1}^{\dagger} a_{2}+a_{1} a_{2}^{\dagger}\right) \\
& +\frac{\hbar}{2} \sqrt{f_{r r} g_{r r}^{o}}\left(\frac{f_{r r^{\prime}}}{f_{r r}}-\frac{g_{r r^{\prime}}^{o}}{g_{r r}^{o}}\right)\left(a_{1}^{\dagger} a_{2}^{\dagger}+a_{1} a_{2}\right) .
\end{aligned}
$$

This Hamiltonian does not preserve the total number of quanta (polyad), and consequently the dimension of its matrix representation is infinite. In practice, a polyad preserving Hamiltonian is considered

$$
\hat{H}=\omega_{\operatorname{loc}} \sum_{i=1}^{2}\left(a_{i}^{\dagger} a_{i}+a_{i} a_{i}^{\dagger}\right)+\lambda_{\operatorname{loc}}\left(a_{1}^{\dagger} a_{2}+a_{1} a_{2}^{\dagger}\right),
$$

where we have introduced the definitions

$$
\begin{aligned}
& \omega_{\mathrm{loc}}=\frac{\hbar}{2} \sqrt{f_{r r} g_{r r}^{o}}, \\
& \lambda_{\mathrm{loc}}=\frac{\hbar}{2} \sqrt{f_{r r} g_{r r}^{o}}\left(\frac{f_{r r^{\prime}}}{f_{r r}}+\frac{g_{r r^{\prime}}^{o}}{g_{r r}^{o}}\right) .
\end{aligned}
$$

It should be clear that the Hamiltonian (57) is expected to provide a good description as long as the polyad breaking term in (56) is neglected. In this section we will see that it is possible to work with a Hamiltonian of type (57) to describe systems where the full Hamiltonian (56) is expected to be considered.

Let us now introduce the symmetry-adapted coordinates in the Hamiltonian (54)

$$
S_{g}=\frac{1}{2}\left(q_{1}+q_{2}\right), \quad S_{u}=\frac{1}{2}\left(q_{1}-q_{2}\right),
$$

with the corresponding induced transformation in the momenta. In the new coordinates the Hamiltonian transforms into two independent harmonic oscillators (normal modes)

$$
\hat{H}=\frac{1}{2}\left(G_{g g} P_{g}^{2}+G_{u u} P_{u}^{2}\right)+\frac{1}{2}\left(F_{g g} S_{g}^{2}+F_{u u} S_{u}^{2}\right),
$$

where the following definitions are introduced:

$$
\begin{array}{ll}
G_{g g}=g_{r r}^{o}+g_{r r^{\prime}}^{o}, & G_{u u}=g_{r r}^{o}-g_{r r^{\prime}}^{o}, \\
F_{g g}=f_{r r}+f_{r r^{\prime}}, & F_{u u}=f_{r r}-f_{r r^{\prime}} .
\end{array}
$$

An algebraic representation is obtained through the introduction of the bosonic realization

$$
a_{\Gamma}^{\dagger}=\alpha_{\Gamma} S_{\Gamma}-\frac{i}{2 \hbar \alpha_{\Gamma}} \widehat{\mathcal{P}}_{\Gamma}, \quad a_{\Gamma}=\alpha_{\Gamma} S_{\Gamma}+\frac{i}{2 \hbar \alpha_{\Gamma}} \widehat{\mathcal{P}}_{\Gamma},
$$

where $\Gamma=g, u$ with parameters

$$
\alpha_{g}^{2}=\frac{1}{2 \hbar} \sqrt{\frac{f_{r r}+f_{r r^{\prime}}}{g_{r r}^{o}+g_{r r^{\prime}}^{o}}}, \quad \alpha_{u}^{2}=\frac{1}{2 \hbar} \sqrt{\frac{f_{r r}-f_{r r^{\prime}}}{g_{r r}^{o}-g_{r r^{\prime}}^{o}}} .
$$

The Hamiltonian (60) takes thus the form

$$
\hat{H}=\omega_{g}\left(a_{g}^{\dagger} a_{g}+a_{g} a_{g}^{\dagger}\right)+\omega_{u}\left(a_{u}^{\dagger} a_{u}+a_{u} a_{u}^{\dagger}\right),
$$

with the definitions

$$
\begin{aligned}
& \omega_{g}=\frac{\hbar}{2} \sqrt{\left(f_{r r}+f_{r r^{\prime}}\right)\left(g_{r r}^{o}+g_{r r^{\prime}}^{o}\right)}, \\
& \omega_{u}=\frac{\hbar}{2} \sqrt{\left(f_{r r}-f_{r r^{\prime}}\right)\left(g_{r r}^{o}-g_{r r^{\prime}}^{o}\right)} .
\end{aligned}
$$

The exact connection between the Hamiltonians (56) and (64) is given by the relation between the bosons involved, which is given by

$$
\begin{aligned}
& a_{g}^{\dagger}=\frac{1}{2 \alpha \alpha_{g} \sqrt{2}}\left\{\left(\alpha_{g}^{2}+\alpha^{2}\right)\left(a_{1}^{\dagger}+a_{2}^{\dagger}\right)+\left(\alpha_{g}^{2}-\alpha^{2}\right)\left(a_{1}+a_{2}\right)\right\}, \\
& a_{u}^{\dagger}=\frac{1}{2 \alpha \alpha_{u} \sqrt{2}}\left\{\left(\alpha_{u}^{2}+\alpha^{2}\right)\left(a_{1}^{\dagger}-a_{2}^{\dagger}\right)+\left(\alpha_{u}^{2}-\alpha^{2}\right)\left(a_{1}-a_{2}\right)\right\},
\end{aligned}
$$

where $\alpha^{2}=(2 \hbar)^{-1} \sqrt{f_{r r} / g_{r r}}$. However, we can return to a Hamiltonian of the form (57) introducing the canonical transformation

$$
a_{g}^{\dagger}=\frac{1}{\sqrt{2}}\left(c_{1}^{\dagger}+c_{2}^{\dagger}\right), \quad a_{u}^{\dagger}=\frac{1}{\sqrt{2}}\left(c_{1}^{\dagger}-c_{2}^{\dagger}\right),
$$

where $c_{i}^{\dagger}\left(c_{i}\right)$ are also bosonic operators associated with the $i$ th oscillator. The substitution of (67) into (64) yields

$$
\hat{H}=\omega_{\text {nor }} \sum_{i=1}^{2}\left(c_{i}^{\dagger} c_{i}+c_{i} c_{i}^{\dagger}\right)+\lambda_{\text {nor }}\left(c_{1}^{\dagger} c_{2}+c_{1} c_{2}^{\dagger}\right),
$$

with the definitions

$$
\begin{aligned}
& \omega_{\text {nor }} \\
& \quad=\frac{\hbar}{4}\left(\sqrt{\left(f_{r r}+f_{r r^{\prime}}\right)\left(g_{r r}^{o}+g_{r r^{\prime}}^{o}\right)}+\sqrt{\left(f_{r r}-f_{r r^{\prime}}\right)\left(g_{r r}^{o}-g_{r r^{\prime}}^{o}\right)}\right), \\
& \lambda_{\text {nor }} \\
& \quad=\frac{\hbar}{2}\left(\sqrt{\left(f_{r r}+f_{r r^{\prime}}\right)\left(g_{r r}^{o}+g_{r r^{\prime}}^{o}\right)}-\sqrt{\left(f_{r r}-f_{r r^{\prime}}\right)\left(g_{r r}^{o}-4 g_{r r^{\prime}}^{o}\right)}\right) .
\end{aligned}
$$


The local operators $c_{i}^{\dagger}\left(c_{i}\right)$ do not correspond to the physical local operators $a_{i}^{\dagger}\left(a_{i}\right)$, but their action on an isomorphic local basis may be chosen to be the same. In fact we establish the isomorphism

$$
c_{i}^{\dagger}\left(c_{i}\right) \longleftrightarrow a_{i}^{\dagger}\left(a_{i}\right),
$$

allowing the Hamiltonian (68) to be expressed in the form

$$
\hat{H}=\omega_{\text {nor }} \sum_{i=1}^{2}\left(a_{i}^{\dagger} a_{i}+a_{i} a_{i}^{\dagger}\right)+\lambda_{\text {nor }}\left(a_{1}^{\dagger} a_{2}+a_{1} a_{2}^{\dagger}\right) .
$$

We thus have that the same algebraic Hamiltonian may describe molecules with both local and normal mode behaviors, but the interpretation of the spectroscopic parameters must be considered appropriately in order to obtain the correct physical results for the force constants. This is a remarkable feature, because it implies that both Hamiltonians provide the same fit to experimental energies, but the force constants derived from the optimized spectroscopic parameters are different. We should also note that the correct force constants can be obtained from a local interpretation of the Hamiltonian as long as the polyad breaking term is included. Hence, the use of the Hamiltonian (71) avoids breaking the polyad keeping the correct physical information through the parameters (69). However, this is possible only in the quantum mechanical treatment. The use of coherent states to extract the potential energy surface does not provide the correct results, as we will shortly discuss [53].

The quantitative criterion to choose between the local and the symmetrized description in order to evaluate appropriately the force constants is provided by the connection between (57) and (71), which is obtained by rewriting the spectroscopic parameters in (69) in the form $\sqrt{1+x}$ to carry out the Taylor series expansion with the identifications $x=$ $f_{r r^{\prime}} / f_{r r}$ and $x=g_{r r^{\prime}}^{o} / g_{r r}^{o}$. This analysis leads to the conditions

$$
\left|\frac{f_{r r^{\prime}}}{f_{r r}}\right| \ll 1, \quad\left|\frac{g_{r r^{\prime}}^{o}}{g_{r r}^{o}}\right| \ll 1,
$$

to be able to apply the local mode Hamiltonian (57). In other words,

$$
\lim _{|x| \ll 1} \omega_{\text {nor }}=\omega_{\text {loc }}, \quad \lim _{|x| \ll 1} \lambda_{\text {nor }}=\lambda_{\text {loc }}
$$

We should also stress that in this limit the correspondence $c_{i}^{\dagger}\left(c_{i}\right) \leftrightarrow a_{i}^{\dagger}\left(a_{i}\right)$ is satisfied

$$
\begin{aligned}
& \lim _{|x| \ll 1} a_{g}^{\dagger}=\frac{1}{\sqrt{2}}\left(a_{1}^{\dagger}+a_{2}^{\dagger}\right), \\
& \lim _{|x| \ll 1} a_{u}^{\dagger}=\frac{1}{\sqrt{2}}\left(a_{1}^{\dagger}-a_{2}^{\dagger}\right) .
\end{aligned}
$$

When the conditions (72) are not satisfied the normal mode Hamiltonian (71) should be taken. For the water molecule for instance, we have

$$
\left(\frac{g_{r r^{\prime}}^{o}}{g_{r r}^{o}}\right)^{2} \simeq 2.16 \times 10^{-4}, \quad\left(\frac{f_{r r^{\prime}}}{f_{r r}}\right)^{2} \simeq 3.76 \times 10^{-4}
$$

while for the $\mathrm{CO}_{2}$ molecule

$$
\left(\frac{g_{r r^{\prime}}^{o}}{g_{r r}^{o}}\right)^{2} \simeq 0.326, \quad\left(\frac{f_{r r^{\prime}}}{f_{r r}}\right)^{2} \simeq 0.01,
$$

which are illustrative of local and normal behaviors, respectively.

The Hamiltonians (64) and (71) are completely equivalent, since they provide the same energy spectra with the same force constants. We may now take a further step by applying the anharmonization procedure $[58,59]$

$$
a_{i}^{\dagger} \longrightarrow b_{i}^{\dagger}, \quad a_{i} \longrightarrow b_{i},
$$

in such a way that the Hamiltonian (71) transforms into

$$
\hat{H}=\omega_{\text {nor }} \sum_{i=1}^{2}\left(b_{i}^{\dagger} b_{i}+b_{i} b_{i}^{\dagger}\right)+\lambda_{\text {nor }}\left(b_{1}^{\dagger} b_{2}+b_{1} b_{2}^{\dagger}\right),
$$

which is intended to be diagonalized in the direct product of local Morse oscillators. On the other hand, the Hamiltonian of two interacting Morse oscillators in configuration space takes the form

$$
\hat{H}=\frac{1}{2} g_{r r}^{o} \sum_{i=1}^{2} \hat{p}_{i}+\frac{1}{2} \frac{f_{r r}}{\beta^{2}} \sum_{i=1}^{2} \hat{y}_{i}^{2}+g_{r r^{\prime}}^{o} \hat{p}_{1} \hat{p}_{2}+\frac{f_{r r^{\prime}}}{\beta^{2}} y_{1} y_{2},
$$

whose algebraic representation up to a constant term takes the form

$$
\hat{H}=\omega_{\mathrm{loc}} \sum_{i=1}^{2}\left(b_{i}^{\dagger} b_{i}+b_{i} b_{i}^{\dagger}\right)+\lambda_{\mathrm{loc}}\left(b_{1}^{\dagger} b_{2}+b_{1} b_{2}^{\dagger}\right),
$$

with spectroscopic parameters given by (58). In practice the Hamiltonians (78) and (80) are equivalent, since both provide the same energy spectrum. The difference is given in the relation between the spectroscopic parameters and the structure and force constants. While the anharmonization procedure provides the correct results for the force constants through (78), the treatment in configuration space does not give equivalent results, unless the polyad is broken. Hence, this approach is a consequence of a purely algebraic analysis without analog in the treatments in configuration space. In this paper we will present several applications where this analysis is crucial to obtain the correct force constants.

\section{PES Using Coherent States}

With the advent of algebraic techniques based on unitary groups to describe nuclei $[19,27,28]$, the use of coherent states became a valuable tool to understand the physical content of the models $[51,102]$. The same kind of algebraic techniques were also applied to describe rovibrational spectra of molecules, and, because of the phenomenological feature of models, the analysis of the classical limit through the use of coherent sates was crucial in providing a physical insight into the models [51, 52, 102-105]. According to this method, the diagonal matrix elements of the Hamiltonian in the basis of coherent states provide through a suitable 
transformation a potential energy surface, whose derivatives evaluated at equilibrium give the force constants. In molecular physics however a potential energy surface can be obtained by means of the connection of the spectroscopic parameters with the structure and force constants. A natural question which arises is concerned with the comparison of the force constants provided by both methods. To answer this questions a system of two interacting Morse oscillators will be considered [53].

The quantum mechanical Hamiltonian of two equivalent interacting Morse oscillators in configuration space up to quadratic terms is given by (79). This Hamiltonian may be translated into an algebraic representation by means of the approximated expressions (51), where there the frequency of the oscillators $\omega$ is given by $\omega=\sqrt{f_{r r} / \mu}=\sqrt{f_{r r} g_{r r}^{o}}$. In the expansions (51) all terms of order $1 / \sqrt{k}$ and higher are neglected. The substitution of (51) into the Hamiltonian (79) gives rise to the algebraic realization

$$
\begin{aligned}
\hat{H}= & \frac{\hbar \omega}{2} \sum_{i=1}^{2}\left(\hat{b}_{i}^{\dagger} \hat{b}_{i}+\hat{b}_{i} \hat{b}_{i}^{\dagger}+\frac{1}{2 \kappa}\right)+\lambda\left(\hat{b}_{1}^{\dagger} \hat{b}_{2}+\hat{b}_{1} \hat{b}_{2}^{\dagger}\right) \\
& +\lambda^{\prime}\left(\hat{b}_{1}^{\dagger} \hat{b}_{2}^{\dagger}+\hat{b}_{1} \hat{b}_{2}\right)
\end{aligned}
$$

where

$$
\begin{aligned}
& \lambda=\frac{\hbar \omega}{2}\left(\frac{f_{r r^{\prime}}}{f_{r r}}+\frac{g_{r r^{\prime}}^{o}}{g_{r r}^{o}}\right), \\
& \lambda^{\prime}=\frac{\hbar \omega}{2}\left(\frac{f_{r r^{\prime}}}{f_{r r}}-\frac{g_{r r^{\prime}}^{o}}{g_{r r}^{o}}\right) .
\end{aligned}
$$

The expressions $\omega=\sqrt{f_{r r} g_{r r}^{o}}$. and (82) constitute explicit dependence of general functions

$$
\omega=\omega\left(f_{r r}\right), \quad \lambda=\lambda\left(f_{r r}, f_{r r^{\prime}}\right), \quad \lambda^{\prime}=\lambda^{\prime}\left(f_{r r}, f_{r r^{\prime}}\right),
$$

which will be used in the following discussion. We may interpret the above Hamiltonian as a phenomenological expansion with parameters $\omega, \lambda$, and $\lambda^{\prime}$, and the question which arises is concerned with the possibility to recover the original Hamiltonian from the classical expression obtained through the coherent states. The PES extracted in this way (imposing the condition $p_{1}=p_{2}=0$ ) and denoted by $V(\mathbf{r})$ is given as a function of the parameters. In symbolic form

$$
V=V\left(r_{1}, r_{2} ; \omega, \lambda, \lambda^{\prime}\right) \text {. }
$$

From this expression, the force constants are given by

$$
\begin{gathered}
f_{r r}\left(\omega, \lambda, \lambda^{\prime}\right) \equiv\left(\frac{\partial^{2} V(\mathbf{r})}{\partial r_{1}^{2}}\right)_{0}, \\
f_{r r^{\prime}}\left(\omega, \lambda, \lambda^{\prime}\right) \equiv\left(\frac{\partial^{2} V\left(y_{1}, y_{2}\right)}{\partial r_{1} \partial r_{2}}\right)_{0} .
\end{gathered}
$$

However, we have now the explicit forms (83), and consequently the following consistency relations are to be expected

$$
\begin{aligned}
& f_{r r}\left(\omega\left(f_{r r}\right), \lambda\left(f_{r r}, f_{r r^{\prime}}\right), \lambda^{\prime}\left(f_{r r}, f_{r r^{\prime}}\right)\right)=f_{r r}, \\
& f_{r r^{\prime}}\left(\omega\left(f_{r r}\right), \lambda\left(f_{r r}, f_{r r^{\prime}}\right), \lambda^{\prime}\left(f_{r r}, f_{r r^{\prime}}\right)\right)=f_{r r^{\prime}} .
\end{aligned}
$$

Let us now proceed to consider the coherent states approach. A possible way to express the $S U(2)$ coherent states is the following [53]:

$$
|N ; \alpha\rangle=\frac{1}{\sqrt{N !}} \frac{1}{\sqrt{\left(1+|\zeta|^{2}\right)^{N}}}\left(s^{\dagger}+\zeta t^{\dagger}\right)^{N}|0\rangle,
$$

which may be recast in the form

$$
|N ; \zeta\rangle=\frac{1}{\sqrt{\left(1+|\zeta|^{2}\right)^{N}}} e^{\zeta{\hat{J_{+}}}_{+}}|j,-j\rangle,
$$

where, the operator $\hat{J}_{+}$is given by (33). Using the direct product of two coherent states of the form (88), we obtain the expectation value of the Hamiltonian (81):

$$
\begin{aligned}
\left\langle N ; \zeta_{1} \zeta_{2}|\hat{H}| N ; \zeta_{1} \zeta_{2}\right\rangle= & \frac{\hbar \omega}{\kappa}\left(N+\frac{1}{4}\right)+\frac{\hbar \omega}{\kappa} N(N-1) \\
& \times\left\{\left|\gamma_{1}\right|^{2}+\left|\gamma_{2}\right|^{2}\right\} \\
& +\lambda \frac{N^{2}}{\kappa}\left\{\gamma_{1}^{*} \gamma_{2}+\gamma_{1} \gamma_{2}^{*}\right\} \\
& +\lambda^{\prime} \frac{N^{2}}{\kappa}\left\{\gamma_{1}^{*} \gamma_{2}^{*}+\gamma_{1} \gamma_{2}\right\},
\end{aligned}
$$

where for the sake of simplification, we have introduced the definition

$$
\gamma_{i} \equiv \frac{\zeta_{i}}{\left(1+\left|\zeta_{i}\right|^{2}\right)} .
$$

The following transformation to the classical variables $y$ and $p$ is now proposed [53]:

$$
\begin{aligned}
& \gamma=\frac{\zeta}{\left(1+|\zeta|^{2}\right)}=\frac{1}{\sqrt{2 \kappa}}\left\{\frac{y}{\beta \lambda_{0}}-i \frac{p}{\sqrt{\hbar \omega \mu}}\right\}, \\
& \gamma^{*}=\frac{\zeta^{*}}{\left(1+|\zeta|^{2}\right)}=\frac{1}{\sqrt{2 \kappa}}\left\{\frac{y}{\beta \lambda_{0}}+i \frac{p}{\sqrt{\hbar \omega \mu}}\right\},
\end{aligned}
$$

where

$$
\lambda_{0}=\sqrt{\frac{\hbar}{\omega \mu}} .
$$

It can be proved that (91) reduces to the expected results in the harmonic limit [53]. The substitution of (91) into (89) yields the energy surface

$$
\begin{aligned}
E\left(y_{1}, y_{2}, p_{1}, p_{2}\right)= & \frac{\hbar \omega}{\kappa}\left(N+\frac{1}{4}\right)+\frac{\hbar \omega}{2}\left(\frac{N(N-1)}{k^{2}}\right) \frac{1}{\beta^{2} \lambda_{0}^{2}} \\
& \times\left\{y_{1}^{2}+y_{2}^{2}\right\} \\
& +\frac{\hbar \omega}{2}\left(\frac{N(N-1)}{k^{2}}\right) \frac{1}{\hbar \omega \mu}\left\{p_{1}^{2}+p_{2}^{2}\right\} \\
& +\frac{N^{2}}{\kappa^{2}} \frac{\left(\lambda+\lambda^{\prime}\right)}{\beta^{2} \lambda_{0}^{2}}\left\{y_{1} y_{2}\right\}+\frac{N^{2}}{\kappa^{2}} \frac{\left(\lambda-\lambda^{\prime}\right)}{\hbar \omega \mu} \\
& \times\left\{p_{1} p_{2}\right\},
\end{aligned}
$$


which is a function of the spectroscopic parameters. The expression (93) resembles the original Hamiltonian in configuration space, but we have to substitute the functions (83) in order to prove that the full Hamiltonian is recovered. In fact, taking into account the explicit expressions (82), the energy function (93) transforms into

$$
\begin{aligned}
E\left(y_{1}, y_{2}, p_{1}, p_{2}\right)= & h_{0}+\frac{N(N-1)}{k^{2}} \sum_{i=1}^{2} H_{M}^{(i)}+\frac{N^{2}}{k^{2}} g_{r r^{\prime}}^{o} p_{1} p_{2} \\
& +\frac{N^{2}}{k^{2}} \frac{f_{r r^{\prime}}}{\beta^{2}} y_{1} y_{2}
\end{aligned}
$$

where

$$
h_{0}=\frac{\hbar \omega}{k}\left(N+\frac{1}{4}\right) \text {. }
$$

The original Hamiltonian is recovered in the limit of $N$ large, except for a constant term:

$\lim _{N \rightarrow \infty} E\left(y_{1}, y_{2}, p_{1}, p_{2}\right)=\frac{5}{4} \hbar \omega+\sum_{i=1}^{2} H_{M}^{(i)}+g_{r r^{\prime}}^{o} p_{1} p_{2}+\frac{f_{r r^{\prime}}}{\beta^{2}} y_{1} y_{2}$.

From this expression, the PES is obtained at once by taking the condition $p_{1}=p_{2}=0$,

$$
\begin{aligned}
V\left(y_{1}, y_{2}\right) & =\lim _{N \rightarrow \infty} E\left(y_{1}, y_{2}, p_{1}=0, p_{2}=0\right) \\
& =\frac{f_{r r}}{2 \beta^{2}} y_{i}^{2}+\frac{f_{r r^{\prime}}}{\beta^{2}} y_{1} y_{2},
\end{aligned}
$$

with the consistency result

$$
\begin{aligned}
& \left(\frac{\partial^{2} V\left(y_{1}, y_{2}\right)}{\partial r_{1}^{2}}\right)_{0}=f_{r r}, \\
& \left(\frac{\partial^{2} V\left(y_{1}, y_{2}\right)}{\partial r_{1} \partial r_{2}}\right)_{0}=f_{r r^{\prime}} .
\end{aligned}
$$

In this way the PES has been reproduced [53].

From this analysis one point should be remarked: the reason why it was possible to fully recover the PES, besides having used the appropriate transformation (91), is that the full Hamiltonian (up to quadratic order) was involved. In general this situation is not satisfied, as we next discuss.

7.1. Polyad Approximation. In molecular spectroscopy most of the times an approximation involving polyad conservation is considered as zeroth-order calculation. If necessary a Van Vleck perturbation theory is applied to take into account polyad mixing $[101,106,107]$. In our case of two interacting oscillators the polyad $P=v_{1}+v_{2}$ is considered as a good quantum number, and therefore the corresponding algebraic Hamiltonian to be taken is given by

$$
\hat{H}=\frac{\hbar \omega}{2} \sum_{i=1}^{2}\left(\hat{b}_{i}^{\dagger} \hat{b}_{i}+\hat{b}_{i} \hat{b}_{i}^{\dagger}+\frac{1}{2 \kappa}\right)+\lambda\left(\hat{b}_{1}^{\dagger} \hat{b}_{2}+\hat{b}_{1} \hat{b}_{2}^{\dagger}\right) .
$$

Let us now proceed to analyze the coherent state method to extract the PES. For this Hamiltonian we have

$$
\begin{aligned}
\left\langle N ; \zeta_{1} \zeta_{2}|\hat{H}| N ; \zeta_{1} \zeta_{2}\right\rangle= & \frac{\hbar \omega}{\kappa}\left(N+\frac{1}{4}\right)+\frac{\hbar \omega}{\kappa} N(N-1) \\
& \times\left\{\left|\gamma_{1}\right|^{2}+\left|\gamma_{2}\right|^{2}\right\} \\
& +\lambda \frac{N^{2}}{\kappa}\left\{\gamma_{1}^{*} \gamma_{2}+\gamma_{1} \gamma_{2}^{*}\right\},
\end{aligned}
$$

and applying the transformation (91) we obtain

$$
\begin{aligned}
E\left(y_{1}, y_{2}, p_{1}, p_{2}\right)= & \frac{\hbar \omega}{\kappa}\left(N+\frac{1}{4}\right)+\frac{\hbar \omega}{2}\left(\frac{N(N-1)}{k^{2}}\right) \frac{1}{\beta^{2} \lambda_{0}^{2}} \\
& \times\left\{y_{1}^{2}+y_{2}^{2}\right\} \\
& +\frac{\hbar \omega}{2}\left(\frac{N(N-1)}{k^{2}}\right) \frac{1}{\hbar \omega \mu}\left\{p_{1}^{2}+p_{2}^{2}\right\} \\
& +\frac{N^{2}}{\kappa^{2}} \frac{\lambda}{\beta^{2} \lambda_{0}^{2}}\left\{y_{1} y_{2}\right\}+\frac{N^{2}}{\kappa^{2}} \frac{\lambda}{\hbar \omega \mu}\left\{p_{1} p_{2}\right\} .
\end{aligned}
$$

This expression reproduces the independent Morse oscillators, but it involves only $\lambda$. In this case the consistency relations (86) are not strictly satisfied [53].

If we now impose in the Hamiltonian (101) the constraint $p_{1}=p_{2}=0$, we obtain for the PES

$$
\begin{aligned}
V\left(y_{1}, y_{2} ; \omega, \lambda\right)= & \frac{\hbar \omega}{\kappa}\left(N+\frac{1}{4}\right)+\frac{\hbar \omega}{2}\left(\frac{N(N-1)}{k^{2}}\right) \frac{1}{\beta^{2} \lambda_{0}^{2}} \\
& \times\left\{y_{1}^{2}+y_{2}^{2}\right\}+\frac{N^{2}}{\kappa^{2}} \frac{\lambda}{\beta^{2} \lambda_{0}^{2}}\left\{y_{1} y_{2}\right\} .
\end{aligned}
$$

From this expression we finally obtain for the force constants

$$
\begin{aligned}
& f_{r r}(k, \omega)=\left(\frac{\partial^{2} V\left(y_{1}, y_{2} ; \omega, \lambda\right)}{\partial r_{1}^{2}}\right)_{0}=\left(\frac{N(N-1)}{k^{2}}\right) \frac{\hbar \omega}{\lambda_{0}^{2}}, \\
& f_{r r^{\prime}}(k, \lambda)=\left(\frac{\partial^{2} V\left(y_{1}, y_{2} ; \omega, \lambda\right)}{\partial r_{1} / \partial r_{2}}\right)_{0}=\frac{N^{2}}{\kappa^{2}} \frac{\lambda}{\lambda_{0}^{2}} .
\end{aligned}
$$

If we now take into account the relations for $\omega$ and (82), we arrive at

$$
\begin{aligned}
& \lim _{k \rightarrow \infty} f_{r r}(k, \omega) \equiv \lim _{k \rightarrow \infty}\left(\frac{\partial^{2} V\left(y_{1}, y_{2} ; \omega, \lambda\right)}{\partial r_{1}^{2}}\right)_{0}=f_{r r} \\
& \lim _{k \rightarrow \infty} f_{r r^{\prime}}(k, \lambda) \equiv \lim _{k \rightarrow \infty}\left(\frac{\partial^{2} V\left(y_{1}, y_{2} ; \omega, \lambda\right)}{\partial r_{1} \partial r_{2}}\right)_{0}=f_{r r^{\prime}}
\end{aligned}
$$

as long as the condition $\lambda^{\prime}=0$ is taken into account.

As an example, we next consider the water molecule. A fitting of the vibrational levels of water is a difficult task. 
However, if we consider only the 19 stretching levels (up to polyad 5) reported by Halonen in his contribution in [2], a pretty good fit with an $r m s=0.50 \mathrm{~cm}^{-1}$ is obtained, as long as up to quartic interactions are included (6 parameters involved). Keeping the same energies, but considering the simple Hamiltonian (99), it is not possible to describe the spectrum with a reasonable quality. Reducing the number of energies by considering levels up to polyad 3, a fitting with $\mathrm{rms}=24.23 \mathrm{~cm}^{-1}$ is obtained with the following set of spectroscopic parameters:

$$
(k=60 \longrightarrow N=59), \quad \widetilde{\omega}=3808 \mathrm{~cm}^{-1}, \quad \tilde{\lambda}=-54.64 \mathrm{~cm}^{-1},
$$

where the tilde is introduced to emphasize that the parameters are given in wave numbers. Taking into account the definition (92) and the explicit expressions for the Wilson matrix elements, we obtain for the force constants [53]

$$
\begin{aligned}
f_{r r}(k) & =\left(\frac{N(N-1)}{k^{2}}\right) \frac{\hbar \omega}{\lambda_{0}^{2}}=\left(\frac{N(N-1)}{k^{2}}\right) \frac{(\hbar \omega)^{2}}{\hbar^{2} g_{r r}^{o}} \\
& =\left(\frac{N(N-1)}{k^{2}}\right) 8.0409 \frac{a J}{\AA^{2}}, \\
f_{r r^{\prime}}(k) & =\frac{N^{2}}{\kappa^{2}} \frac{\lambda}{\lambda_{0}^{2}}=\frac{N^{2}}{\kappa^{2}} \frac{\hbar \omega}{\hbar^{2} g_{r r}^{o}} \lambda=-\frac{N^{2}}{\kappa^{2}} 0.1154 \frac{a J}{\AA^{2}} .
\end{aligned}
$$

The force constants can be also calculated from the quantum mechanical Hamiltonian using the connection of the spectroscopic parameters with the force constants given in (82), whose results are displayed in Table 1 with the label QM. In the same table the results (106) are labeled (CS), and $(\operatorname{CS}(N \rightarrow \infty))$ when the limit $N \rightarrow \infty$ is considered. According to the previous analysis the difference between the first column $(\mathrm{QM})$ and the third one $(\mathrm{CS}(N \rightarrow \infty))$ lies on the approximation $\lambda^{\prime}=0$ in the Hamiltonian (81). For $\mathrm{H}_{2} \mathrm{O}$ this parameter is approximately given by $\lambda^{\prime} \approx 7.7810^{-4}$, which when compared with $\lambda \approx-0.0286918$ justify the use of the Hamiltonian (99).

It should be clear that the good agreement shown in Table 1 is due to the fact that $\lambda^{\prime}$ is approximately null, which in turn is satisfied for molecules with local behavior. In other words, the vibrational excitations in water molecule can be approximated as interacting local oscillators. The natural question which arises is concerned with the results in systems with a normal mode behavior.

7.2. Normal Mode Behavior. In this subsection we address the problem of obtaining the PES when a Hamiltonian of type (99) preserving the polyad is considered in the fitting of a spectrum associated with a normal mode behavior. We start with the Hamiltonian (81) in the harmonic limit, which takes the form (56). The corresponding polyad preserving
TABLe 1: Force constants for $\mathrm{H}_{2} \mathrm{O}$ obtained from the fit (118) using (a) the quantum mechanical connection (QM) given by (82), (b) the coherent states method (CS), and (c) the coherent state approach taking the harmonic limit $N \rightarrow \infty$.

\begin{tabular}{lccc}
\hline Force constant & QM & CS & CS $(N \rightarrow \infty)$ \\
\hline$f_{r r}$ aJ $\AA^{-2}$ & 8.0409 & 7.6389 & 8.0409 \\
$f_{r r^{\prime}}$ aJ $\AA^{-2}$ & -0.1122 & -0.1115 & -0.1154 \\
\hline
\end{tabular}

Hamiltonian is given by (57). As we previously discussed, the Hamiltonian (56) in configuration space is given by

$$
\hat{H}=\frac{1}{2} g_{r r}^{o}\left(p_{1}^{2}+p_{2}^{2}\right)+\frac{1}{2} f_{r r}\left(q_{1}^{2}+q_{2}^{2}\right)+g_{r r^{\prime}}^{o} p_{1} p_{2}+f_{r r^{\prime}} q_{1} q_{2},
$$

which can be transformed into the algebraic representation (64), when symmetry-adapted coordinates are introduced. The exact connection between the Hamiltonians (56) and (64) is given by the relation between the bosons involved (66), but we can return to the Hamiltonian of the form (56) introducing the canonical transformation (67). The substitution of (67) into (64) yields (68). As we stressed, the local operators $c_{i}^{\dagger}\left(c_{i}\right)$ do not correspond to the physical local operators $a_{i}^{\dagger}\left(a_{i}\right)$, but their action on an isomorphic local basis may be chosen to be the same. In fact we will establish the isomorphism $c_{i}^{\dagger}\left(c_{i}\right) \leftrightarrow a_{i}^{\dagger}\left(a_{i}\right)$, allowing the Hamiltonian (68) to be expressed in the form

$$
\hat{H}=\omega_{\text {nor }} \sum_{i=1}^{3}\left(a_{i}^{\dagger} a_{i}+a_{i} a_{i}^{\dagger}\right)+\lambda_{\text {nor }} \sum_{i>j}\left(a_{i}^{\dagger} a_{j}+a_{i} a_{j}^{\dagger}\right) .
$$

We may now consider the anharmonization procedure [58, 59]

$$
a_{i}^{\dagger} \longrightarrow b_{i}^{\dagger}, \quad a_{i} \longrightarrow b_{i}
$$

to obtain the Hamiltonian (78)

$$
\hat{H}=\omega_{\text {nor }} \sum_{i=1}^{2}\left(b_{i}^{\dagger} b_{i}+b_{i} b_{i}^{\dagger}\right)+\lambda_{\text {nor }}\left(b_{1}^{\dagger} b_{2}+b_{1} b_{2}^{\dagger}\right) .
$$

This approach of identification of the spectroscopic parameters in terms of the structure and force constants does not have analog in the treatments in configuration space.

As an example of a normal mode behavior, we will consider the molecule of carbon dioxide. Although the strong Fermi interaction of this molecule makes it inappropriate to use the Hamiltonian (99) to obtain good values of force constants, we will proceed to estimate them by taking the relevant stretching parameters from a fit using the $U(2) \times$ $U(3) \times U(2)$ model given in [68], where the purely stretching Hamiltonian up to quadratic terms coincides with (99). The parameters are given by

$$
(k=160, N=159), \quad \widetilde{\omega}=916.15 \mathrm{~cm}^{-1}, \quad \tilde{\lambda}=-474.22 \mathrm{~cm}^{-1} .
$$


TABLE 2: Force constants for $\mathrm{CO}_{2}$ by means of (a) [68] (QM*), (b) using the quantum mechanical connection (QM) given by (110), (c) the coherent states method (CS) using (103), (d) the coherent state approach taking the harmonic limit $N \rightarrow \infty$, and (e) the coherent states approach using the Hamiltonian (81), but estimated through the connection with the Hamiltonian (110) (CS-PB).

\begin{tabular}{lccccc}
\hline Force constant & QM $^{*}$ & QM & CS & CS $(N \rightarrow \infty)$ & CS-PB \\
\hline$f_{r r}$ aJ $\AA^{(-2)}$ & 15.55 & 17.48 & 13.32 & 13.58 & 17.48 \\
$f_{r r^{\prime}}$ aJ $\AA^{(-2)}$ & 1.86 & 6.48 & -3.46 & -3.51 & 6.48 \\
\hline
\end{tabular}

If we intend to calculate the force constants by means of (103) according to our coherent states method, we obtain the results displayed in the columns CS and CS $(N \rightarrow \infty)$ of Table 2. In contrast, when the force constants are calculated quantum mechanically using (69) the results of column labeled QM is obtained. As a reference, the force constants obtained from [68] by means of the $U(2) \times U(3) \times U(2)$ model are displayed in the column labeled $Q M^{*}$. In the latter case the bending interactions are involved in the calculations which explains the difference. Since in this work we are interested in evaluating the coherent states method, we will be interested in comparing the predicted force constants with the column $Q M$.

While for $f_{r r}$ the values are not too different, in the case of the force constant $f_{r r^{\prime}}$ the results are fairly different even qualitatively. We should stress the wrong sign of the force constant $f_{r r^{\prime}}$, which is determined by the sign of $\lambda$ according to (103). This result is a consequence of having used a polyad preserving Hamiltonian to obtain the PES. This assertion can be proved by means of the criterion (76), which reflects the normal behavior that characterizes this molecule. Hence, it is clear that for carbon dioxide the coherent state method fails when a polyad preserving Hamiltonian is used. In molecules with normal mode behavior, the appropriate Hamiltonian to recover the PES must be the full Hamiltonian (81), which means that in principle the polyad has to be broken. In other words, in this case the parameter $\lambda^{\prime}$ in (81) is not negligible if one intends to calculate the force constants. In fact for $\mathrm{CO}_{2}$ we have

$$
\frac{\lambda^{\prime}}{\lambda}=\frac{f_{r r^{\prime}} / f_{r r}-g_{r r^{\prime}}^{o} / g_{r r}^{o}}{f_{r r^{\prime}} / f_{r r}+g_{r r^{\prime}}^{o} / g_{r r}^{o}} \approx-1.42 .
$$

From the previous analysis we know that the coherent state approach applied to the full Hamiltonian (81) taking the limit $k \rightarrow \infty$ provides the same force constants that the quantum mechanical result labeled QM in Table 2. This is a remarkable result that must be taken into account when using this treatment. To emphasize this point in Table 2, a column labeled as coherent state-polyad breaking (CS-PB) has been added, whose results are expected to be obtained from a fit using (81).

We have thus presented the method of coherent states to extract the PES of molecular systems in the context of the Born-Oppenheimer approximation. The analysis has been presented for the one-dimensional version of the algebraic models based on unitary groups. In particular the algebraic representation of two interacting Morse oscillators was considered. Special attention was paid to the case when the polyad is considered as a good quantum number. It was shown that in this approximation the coherent states approach reproduce the PES only for systems with local mode behavior. For molecules with a normal mode behavior the coherent state approach is still valid, but a Hamiltonian breaking the polyad must be considered, as a consequence of the strong coupling. The two independent oscillators stop being a good zeroth-order Hamiltonian, and the parameter $\omega$ in (81) is strongly influenced by the interaction associated with the parameter $\lambda^{\prime}$. Since the polyad concept is crucial to be able to carry out the calculations in the description vibrational excitations in molecules, we conclude that in practice the suitability of the coherent state approach is restricted to molecules with local mode behavior. In the next sections we proceed to show how to obtain the PES quantum mechanically for different representative systems of semirigid molecules.

\section{Application to Water Molecule}

In an algebraic description, where the polyad is considered as a pseudo-quantum number, the possible force constants to be estimated from a fit are restricted. The reason is that many terms should be taken away from the Hamiltonian, eliminating the possibility of calculating the corresponding force constants. When the linear approximation (51) is considered, a similar situation is present. In this section we will show that, when the quadratic terms are included in the expansions of the coordinates and momenta (see (48) and (49)), the whole set of force constants can be estimated up to the order considered in the Hamiltonian. To this end we will present the vibrational description of $\mathrm{H}_{2}{ }^{16} \mathrm{O}$ in the framework of the $S U(2)$ model, the one-dimensional case of the unitary group approach.

To estimate the PES of $\mathrm{H}_{2}{ }^{16} \mathrm{O}$ beyond the linear approximation (51), a tensorial formalism is developed to expand the Hamiltonian in powers of $1 / \sqrt{k}$ in terms of symmetryadapted operators, which in turn are given in terms of local creation and destruction Morse operators in the spirit to establish the connection between the effective Hamiltonian approach and the standard local mode models in configuration space [58]. Hence, in the framework of the algebraic model, independent Morse oscillators are considered as a zeroth-order approximation. In a Morse oscillator basis, however, the interaction terms couple the whole space in the Hamiltonian matrix, not allowing to take advantage of the simplifications brought about by approximately conserved polyad numbers. This problem, however, can be avoided by keeping only the terms which preserve the polyad. Here we will first proceed to obtain the Hamiltonian in terms of the Morse variables $y_{i}$ and their momenta, to thereafter carry out their expansions in terms of the symmetry-adapted operators, keeping the terms preserving the polyad. A remarkable consequence of keeping the terms of order $(1 / \sqrt{\kappa})$ in the expansion in the Morse coordinates and momenta is that all the force constants up to quartic terms in the potential can be determined, even with the constraint of the polyad as a good quantum number, as previously mentioned. 
The equilibrium structure of the water molecule is nonlinear with structure parameters $r_{\mathrm{OH}}=0.9575 \AA$ and $\angle \mathrm{HOH}=104.51^{\circ}$ [108]. The point symmetry is $\mathcal{C}_{2 v}$, but it is enough to consider the subgroup $\mathcal{C}_{2}$ since the vibrations take place on a plane. This molecule has three degrees of freedom, two of them associated with the stretching modes $(A \oplus B)$ and the other to the bending mode $(A)$. The harmonic approximation provides a complete basis in terms of normal modes which can be used to diagonalize a general Hamiltonian. In the standard notation this basis is labeled by $\left|\nu_{1} \nu_{2} \nu_{3}\right\rangle$, where $\nu_{1}$ and $\nu_{3}$ are the number of quanta in the stretching $A$ and $B$ modes, respectively, while $\nu_{2}$ is associated with the bending $A$ mode [8].

If we use internal displacement coordinates, the quantum mechanical Hamiltonian that describes the vibrational excitations of the $\mathrm{H}_{2} \mathrm{O}$ molecule takes the form (14)

$$
H=\frac{1}{2} \widetilde{\mathbf{p}} G(q) \hat{\mathbf{p}}+V(\mathbf{q}) .
$$

Here we have omitted the purely quantum mechanical term derived from the kinetic energy not involving momentum operators. The components $q_{r}$ and $q_{r^{\prime}}$ will be assigned to the $\mathrm{O}-\mathrm{H}$ stretching displacements coordinates from the equilibrium, while $q_{\phi}$ corresponds to the displacement from equilibrium of the $\angle \mathrm{HOH}$-bending coordinate. In this way we have

$$
q_{r}=\Delta r, \quad q_{\phi}=r_{e} \Delta \phi, \quad q_{r^{\prime}}=\Delta r^{\prime},
$$

where $r_{e}$ has been added in the definition of the bending coordinate in order to have the same distance units. For semirigid molecules a reasonable approach consists in expanding both the $\mathbf{G}(\mathbf{q})$ matrix as well as the potential $V(\mathbf{q})$ in a Taylor series about the equilibrium configuration. It has been pointed out, however, that, in order to obtain convergence for large oscillations, the Morse variables $y_{i}=1-e^{-\beta_{i} q_{i}}$ are appropriate [109-114]. Hence, here we consider an expansion of the variables $\left\{y_{i}, i=r, r^{\prime}, \phi\right\}$ for both stretching and bending coordinates. In this spirit the elements $g_{i j}$ of the $\mathbf{G}(\mathbf{q})$ matrix are expanded up to quadratic terms, since we intend to consider an expansion in the Hamiltonian up to quartic terms. Consequently an expansion up to quartic terms is carried out for the potential. It is worth noting the relevance of the ratio $y_{i} / \beta_{i}$. In the rigid limit this ratio tends to the variable $q_{i}$. The zeroth-order Hamiltonian is then given by

$$
\hat{H}_{0}=\hat{H}_{r}^{M}+\hat{H}_{r^{\prime}}^{M}+\hat{H}_{\phi}^{M},
$$

where

$$
\hat{H}_{i}^{M}=\frac{1}{2} g_{i i}^{o} \hat{p}_{i}^{2}+\frac{1}{2} f_{i i} y_{i}^{2}, \quad i=r, r^{\prime}, \phi,
$$

while the complete Hamiltonian up to quartic terms takes the form [58]

$$
\begin{aligned}
\hat{H}= & \hat{H}_{0}+\left(g_{r r^{\prime}}\right)_{0} \hat{p}_{r} \hat{p}_{r^{\prime}}+\frac{1}{2}\left(\frac{\partial g_{\phi \phi}}{\partial q_{r}}\right)_{0} \beta_{r}^{-1}\left(y_{r}+y_{r^{\prime}}\right) \hat{p}_{\phi}^{2} \\
& +\left(\frac{\partial g_{r \phi}}{\partial q_{\phi}}\right)_{0} \beta_{\phi}^{-1}\left(\hat{p}_{r}+\hat{p}_{r^{\prime}}\right) \frac{\left(y_{\phi} \hat{p}_{\phi}+\hat{p}_{\phi} y_{\phi}\right)}{2}
\end{aligned}
$$

$$
\begin{aligned}
& +\frac{1}{4}\left[\left(\frac{\partial^{2} g_{\phi \phi}}{\partial q_{r}^{2}}\right)_{0}+\left(\frac{\partial g_{\phi \phi}}{\partial q_{r}}\right)_{0} \beta_{r}\right] \beta_{r}^{-2}\left(y_{r}^{2}+y_{r^{\prime}}^{2}\right) \hat{p}_{\phi}^{2} \\
& -\frac{1}{2}\left(\frac{\partial^{2} g_{\phi \phi}}{\partial q_{\phi}^{2}}\right)_{0} \beta_{r}^{-2} y_{r} y_{r^{\prime}} p_{\phi}^{2} \\
& +\frac{1}{4}\left[\left(\frac{\partial^{2} g_{\phi \phi}}{\partial q_{\phi}^{2}}\right)_{0}+\left(\frac{\partial g_{\phi \phi}}{\partial q_{\phi}}\right)_{0} \beta_{\phi}\right] \beta_{\phi}^{-2} \hat{p}_{\phi} y_{\phi}^{2} \hat{p}_{\phi} \\
& +\frac{1}{2}\left[\left(\frac{\partial^{2} g_{r r^{\prime}}}{\partial q_{\phi}^{2}}\right)_{0}+\left(\frac{\partial q_{r r^{\prime}}}{\partial q_{\phi}}\right)_{0} \beta_{\phi}\right] \beta_{\phi}^{-2} \hat{p}_{r} \hat{p}_{r^{\prime}} y_{\phi}^{2} \\
& +\left(g_{r \phi}\right)_{0}\left(\hat{p}_{r}+\hat{p}_{r^{\prime}}\right) \hat{p}_{\phi} \\
& +\frac{1}{2}\left(\frac{\partial g_{\phi \phi}}{\partial q_{\phi}}\right)_{0} \beta_{\phi}^{-1} \hat{p}_{\phi} y_{\phi} \hat{p}_{\phi} \\
& +\left(\frac{\partial g_{r r^{\prime}}}{\partial q_{\phi}}\right)_{0} \beta_{\phi}^{-1} \hat{p}_{r} y_{\phi} \hat{p}_{r^{\prime}} \\
& +\left(\frac{\partial g_{r \phi}}{\partial q_{r^{\prime}}}\right)_{0} \beta_{r}^{-1}\left(\hat{p}_{r} y_{r^{\prime}}+\hat{p}_{r^{\prime}} y_{r}\right) \hat{p}_{\phi} \\
& +\frac{1}{4}\left(\frac{\partial^{2} g_{\phi \phi}}{\partial q_{\phi}^{2}}\right)_{0} \beta_{r}^{-1} \beta_{\phi}^{-1}\left(\hat{p}_{r} y_{r^{\prime}}+\hat{p}_{r^{\prime}} y_{r}\right)\left(y_{\phi} \hat{p}_{\phi}+\hat{p}_{\phi} y_{\phi}\right) \\
& +\frac{1}{2}\left(\frac{\partial^{2} g_{\phi \phi}}{\partial q_{r} \partial q_{\phi}}\right)_{0} \beta_{r}^{-1}\left(y_{r}+y_{r^{\prime}}\right) \hat{p}_{\phi} y_{\phi} \hat{p}_{\phi} \\
& +\frac{1}{2}\left[\left(\frac{\partial^{2} q_{r \phi}}{\partial q_{r}^{2}}\right)_{0}+\left(\frac{\partial^{2} g_{r \phi}}{\partial q_{r}}\right)_{0} \beta_{r}\right] \beta_{r}^{-2}\left(\hat{p}_{r} y_{r^{\prime}}^{2}+\hat{p}_{r^{\prime}} y_{r}^{2}\right) \hat{p}_{\phi} \\
& +\frac{1}{2}\left[\left(\frac{\partial^{2} g_{r \phi}}{\partial q_{\phi}^{2}}\right)_{0}+\left(\frac{\partial g_{r \phi}}{\partial q_{\phi}}\right)_{0} \beta_{\phi}\right] \beta_{\phi}^{-2}\left(\hat{p}_{r}+\hat{p}_{r^{\prime}}\right) \\
& \times \frac{\left(y_{\phi}^{2} \hat{p}_{\phi}+\hat{p}_{\phi} y_{\phi}^{2}\right)}{2}+f_{r r^{\prime}} \beta_{r}^{-2} y_{r} y_{r^{\prime}} \\
& +\frac{1}{2}\left(\beta_{\phi} f_{r \phi}+f_{r \phi \phi}\right) \beta_{r}^{-1} \beta_{\phi}^{-2}\left(y_{r}+y_{r^{\prime}}\right) y_{\phi}^{2} \\
& +\frac{1}{4 !}\left(11 \beta_{\phi}^{2} f_{\phi \phi}+6 \beta_{\phi} f_{\phi \phi \phi}+f_{\phi \phi \phi \phi}\right) \beta_{\phi}^{-4} y_{\phi}^{4} \\
& +\frac{1}{4 !}\left(11 \beta_{r}^{2} f_{r r}+6 \beta_{r} f_{r r r}+f_{r r r r}\right) \beta_{r}^{-4}\left(y_{r}^{4}+y_{r^{\prime}}^{4}\right) \\
& +\frac{1}{4 !} 6\left(\beta_{r} \beta_{\phi} f_{r \phi}+\beta_{\phi} f_{r r \phi}+\beta_{r} f_{r \phi \phi}+f_{r r \phi \phi}\right) \\
& \times \beta_{r}^{-2} \beta_{\phi}^{-2}\left(y_{r}^{2}+y_{r^{\prime}}^{2}\right) y_{\phi}^{2} \\
& +\frac{1}{4 !} 12\left(\beta_{\phi} f_{r r^{\prime} \phi}+f_{r r^{\prime} \phi \phi}\right) \beta_{r}^{-2} \beta_{\phi}^{-2} y_{r} y_{r^{\prime}} y_{\phi}^{2} \\
& +\frac{1}{4 !} 6\left(\beta_{r}^{2} f_{r r^{\prime}}+2 \beta_{r} f_{r r^{\prime} r^{\prime}}+f_{r r r^{\prime} r^{\prime}}\right) \beta_{r}^{-4} y_{r}^{2} y_{r^{\prime}}^{2} \\
& +\frac{1}{4 !} 4\left(2 \beta_{r}^{2} f_{r r^{\prime}}+3 \beta_{r} f_{r r r^{\prime}}+f_{r r r r^{\prime}}\right) \beta_{r}^{-4}\left(y_{r^{\prime}} y_{r}^{3}+y_{r} y_{r^{\prime}}^{3}\right)
\end{aligned}
$$




$$
\begin{aligned}
& +f_{r \phi} \beta_{r}^{-1} \beta_{\phi}^{-1}\left(y_{r}+y_{r^{\prime}}\right) y_{\phi} \\
& +\frac{1}{6}\left(3 \beta_{r} f_{r r}+f_{r r r}\right) \beta_{r}^{-3}\left(y_{r}^{3}+y_{r^{\prime}}^{3}\right) \\
& +\frac{1}{6}\left(3 \beta_{\phi} f_{\phi \phi}+f_{\phi \phi \phi}\right) \beta_{\phi}^{-3} y_{\phi}^{3} \\
& +\frac{1}{2}\left(\beta_{r} f_{r r^{\prime}}+f_{r r r^{\prime}}\right) \beta_{r}^{-3}\left(y_{r^{\prime}} y_{r}^{2}+y_{r} y_{r^{\prime}}^{2}\right) \\
& +\frac{1}{2}\left(\beta_{r} f_{r \phi}+f_{r r \phi}\right) \beta_{\phi}^{-1} \beta_{r}^{-2}\left(y_{r}^{2}+y_{r^{\prime}}^{2}\right) y_{\phi} \\
& +f_{r r^{\prime} \phi} \beta_{r}^{-2} \beta_{\phi} y_{r} y_{r^{\prime}} y_{\phi} \\
& +\frac{1}{4 !} 4\left(2 \beta_{r}^{2} f_{r \phi}+3 \beta_{r} f_{r r \phi}+f_{r r r \phi}\right) \beta_{r}^{-3} \beta_{\phi}^{-1}\left(y_{r}^{3}+y_{r^{\prime}}^{3}\right) y_{\phi} \\
& +\frac{1}{4 !} 4\left(2 \beta_{\phi}^{2} f_{r \phi}+3 \beta_{\phi} f_{r \phi \phi}+f_{r \phi \phi \phi}\right) \beta_{\phi}^{-3} \beta_{r}^{-1}\left(y_{r}+y_{r^{\prime}}\right) y_{\phi}^{3} \\
& +\frac{1}{4 !} 12\left(\beta_{r} f_{r r^{\prime} \phi}+f_{r r^{\prime} r^{\prime} \phi}\right) \beta_{r}^{-3} \beta_{\phi}^{-1}\left(y_{r} y_{r^{\prime}}^{2}+y_{r^{\prime}} y_{r}^{2}\right) y_{\phi} .
\end{aligned}
$$

Since we consider noninteracting Morse oscillators as the zeroth-order Hamiltonian, the natural basis to diagonalize the Hamiltonian (117) consists in the direct product of Morse functions

$$
\left|j_{r}, j_{\phi} ; v_{r}, v_{r^{\prime}}, v_{\phi}\right\rangle=\left|j_{r} v_{r}\right\rangle \otimes\left|j_{\phi} v_{\phi}\right\rangle \otimes\left|j_{r} v_{r^{\prime}}\right\rangle,
$$

where $v_{r}$ and $v_{r^{\prime}}$ stand for the number of quanta associated to $q_{r}$ and $q_{r^{\prime}}$, respectively, $v_{\phi}$ corresponds to the bending $q_{\phi}$ coordinate, and we have taken into account the equivalence of the stretching bonds through the equality $j_{r}=j_{r^{\prime}}$. To simplify the matrix representation of the Hamiltonian, the polyad should be introduced. The polyad is established from the resonances of the first overtones with the fundamentals. For $\mathrm{H}_{2} \mathrm{O}$ the energy of the symmetric mode is approximately twice the bending energy [115]. Hence, the appropriate polyad number is defined by

$$
P=2\left(v_{r}+v_{r^{\prime}}\right)+v_{\phi}=2\left(\nu_{1}+v_{3}\right)+v_{2} .
$$

We should note that $P$ can be written in terms of both local and normal quantum numbers. This situation is due to the fact that this molecule presents a strong local behavior. In cases where a normal behavior appears, the polyad can be given only in terms of normal quantum numbers. An example of this case is the $\mathrm{BF}_{3}$, a molecule that will be discussed in the next section.

Although we may use the basis (118) to diagonalize the Hamiltonian, it is more convenient to propose a symmetryadapted basis. The new basis should carry quantum numbers isomorphic to the normal quanta, as well as irreducible representations of the symmetry group. A basis with these characteristics can be constructed in several ways, but the most efficient approach consists in a combination of the eigenfunction method developed by Chen [88] and the diagonalization of the symmetry-adapted number operators in a harmonic basis. This approach is explained in detail in $[89,90]$. The Hamiltonian, on the other hand, being totally symmetric, can be expressed in terms of symmetryadapted tensors coupled to the totally symmetric irreducible representation. Hence, we introduce the following tensors [58]:

$$
\begin{gathered}
\hat{y}_{0, x}^{\Gamma}=\sum_{i} \alpha_{x, i}^{\Gamma} f_{v_{i}}^{d}, \\
\hat{y}_{1, x}^{\Gamma \dagger}=\sum_{i} \alpha_{x, i}^{\Gamma} f_{v_{i}} \hat{b}_{i}^{\dagger}, \\
\hat{y}_{2, x}^{\Gamma \dagger}=\sum_{i} \alpha_{x, i}^{\Gamma} g_{v_{i}} \hat{b}_{i}^{\dagger} \hat{b}_{i}^{\dagger},
\end{gathered}
$$

where the subindex $x=s, b$ was included in order to distinguish tensors arising from the stretching and bending degrees of freedom, respectively, and the coefficients $\alpha_{x, i}^{\Gamma, \gamma}$ correspond to the coefficients of the symmetry-adapted functions of one quantum.

When the expressions (48) and (49) are substituted into (117), each contribution of the Hamiltonian will be given in terms of an expansion in powers of the parameter $1 / \sqrt{\kappa}$, which, according to the approximation, ranges from $(1 / \sqrt{\kappa})^{0}$ to $1 / \kappa^{2}$. If we restrict ourselves to keep only the polyad preserving terms, several contributions disappear. Given this substitution, we now invoke the symmetry-adapted tensors (120) in order to make clear the invariance of the Hamiltonian as well as the order of the interactions. Hence, in terms of symmetry-adapted tensors, the Hamiltonian (117) takes an expansion of the form

$$
\begin{aligned}
\hat{H}= & \hat{H}_{0}-x_{1}\left[\left(y_{1, s}^{A \dagger} y_{1, s}^{A}-y_{1, s}^{B \dagger} y_{1, s}^{B}\right)\right] \\
& -x_{2} \sqrt{2}\left[y_{1, s}^{A \dagger} y_{1, b}^{A} y_{1, b}^{A}+\frac{1}{\sqrt{\kappa}} y_{0, s}^{A} y_{1, b}^{A \dagger} y_{1, b}^{A}+h c\right] \ldots,
\end{aligned}
$$

whose complete expression is given in [58]. In [58] the Hamiltonian is expressed in terms of two kinds of tensors associated with the coordinates and momentum. The reason is that the coefficients involved in the expansions of (48) and (49) were written in different ways. The coefficients associated with the kinetic energy are named $x_{i}$, while the ones associated with the potential expansion are $z_{i}$. They are given in Tables 1 and 2 of [58]. In spite of the apparent complexity of the Hamiltonian (121), the form of the tensorial expansion is very enlightening. On one hand it makes clear the order of the interaction involved in each term, and on the other hand provides the type of additional interactions which are taken into account when the polyad is preserved. If in (121) we carry out the limit $\kappa \rightarrow \infty$, all the tensors $\hat{y}_{0, x}^{\Gamma}$ and $\hat{y}_{2, x}^{\Gamma \dagger}$ vanish and consequently some of the coefficients disappear. As a consequence only the subset of force constants $\left\{f_{r r}, f_{\phi \phi}, f_{r r^{\prime}}, f_{r r r r}, f_{r r r r^{\prime}}, f_{r r r^{\prime} r^{\prime}}, f_{\phi \phi \phi \phi}, f_{r r \phi \phi}, f_{r \phi \phi}, f_{r r^{\prime} \phi \phi}\right\}$ are able to be determined. These force constants are the ones that are determined in a traditional calculation using the linear expansions (51) preserving the polyad [57]. In contrast, in this approach all the force constants up to quartic order are determined. In the same spirit we may break the polyad taking terms up to order $1 / \sqrt{\kappa}[101]$. 
TABLE 3: Force constants for $\mathrm{H}_{2} \mathrm{O}$. In the first column is indicated the order that should be considered in order to obtain the corresponding force constant.

\begin{tabular}{|c|c|c|c|c|c|c|}
\hline Order & Force constant & This work $^{\mathrm{a}}$ & Jensen $^{\mathrm{b}}$ & Halonen and Carrington Jr. ${ }^{c}$ & Lemus et al. $^{\mathrm{d}}$ & Ab initio $^{\mathrm{e}}$ \\
\hline 1 & $\beta_{s} / \AA^{-1}$ & 2.1573 & & 2.053 & 2.1542 & \\
\hline 1 & $D_{s} / \mathrm{aJ}$ & 0.8694 & & 0.999 & 0.9050 & \\
\hline 1 & $\beta_{b} / \AA^{-1}$ & 0.8279 & & & 0.7296 & \\
\hline 1 & $D_{b} / \mathrm{aJ}$ & 0.5190 & & & 0.7055 & \\
\hline 1 & $f_{r r} / \mathrm{aJ} \AA^{-2}$ & 8.093 & 8.4393 & 8.428 & 8.401 & 8.443 \\
\hline 1 & $r_{e}^{2} f_{\phi \phi} / \mathrm{aJ}$ & 0.652 & 0.7070 & 0.699 & 0.688 & 0.7921 \\
\hline 1 & $f_{r r^{\prime}} / \mathrm{aJ} \AA^{-2}$ & -0.1571 & -0.1051 & -0.101 & -0.11 & -0.100 \\
\hline $1 / \sqrt{\kappa}$ & $r_{e} f_{r \phi} / \mathrm{aJ} \AA^{-1}$ & -1.0578 & 0.3064 & 0.219 & & 0.2743 \\
\hline $1 / \sqrt{\kappa}$ & $f_{r r r} / \mathrm{aJ} \AA^{-3}$ & -35.880 & -55.40 & -51.91 & & -56.400 \\
\hline $1 / \sqrt{\kappa}$ & $r_{e} f_{r r^{\prime}} / \mathrm{aJ} \AA^{-2}$ & -3.098 & -0.447 & 0.414 & & -0.505 \\
\hline $1 / \sqrt{\kappa}$ & $f_{r r r^{\prime} \phi} / \mathrm{aJ} \AA^{-3}$ & -0.358 & -0.318 & 0.645 & & -0.076 \\
\hline 1 & $r_{a}^{2} f_{r \phi \phi} / \mathrm{aJ} \AA^{-1}$ & -2.6243 & -0.3383 & -0.314 & -0.51 & -0.3210 \\
\hline $1 / \sqrt{\kappa}$ & $r_{e} f_{r r \phi} / \mathrm{aJ} \AA^{-2}$ & 21.442 & -0.252 & 1.341 & & -0.084 \\
\hline $1 / \sqrt{\kappa}$ & $r_{e}^{3} f_{\phi \phi \phi} / \mathrm{aJ}$ & -8.4312 & -0.7332 & -0.918 & & -0.7482 \\
\hline 1 & $f_{r r r r} / \mathrm{aJ} \AA^{-4}$ & 130.87 & 306.0 & 248.7 & 275.39 & 338 \\
\hline 1 & $r_{e}^{2} f_{r r \phi \phi} / \mathrm{aJ} \AA^{-2}$ & -15.89 & -0.950 & -2.0 & -15.699 & -0.28 \\
\hline 1 & $f_{r r r r^{\prime}} / \mathrm{aJ} \AA^{-4}$ & 7.861 & 2.57 & & 3.728 & -0.30 \\
\hline 1 & $r_{e}^{2} f_{r r^{\prime} \phi \phi} / \mathrm{aJ} \AA^{-2}$ & 2.3910 & 0.1150 & & -0.632 & 0.62 \\
\hline 1 & $f_{r r r^{\prime} r^{\prime}} / \mathrm{aJ} \AA^{-4}$ & -9.843 & 1.93 & & -12.68 & 0.52 \\
\hline 1 & $r_{e}^{4} f_{\phi \phi \phi \phi} / \mathrm{aJ}$ & 23.349 & -0.238 & -0.1 & 2.32 & -0.74 \\
\hline $1 / \sqrt{\kappa}$ & $r_{e} f_{r r r \phi} / \mathrm{aJ} \AA^{-3}$ & -100.98 & -6.14 & & & -1.2 \\
\hline $1 / \sqrt{\kappa}$ & $r_{e} f_{r r r^{\prime} \phi} / \mathrm{aJ} \AA^{-3}$ & 3.5584 & -3.22 & & & 0.2 \\
\hline $1 / \sqrt{\kappa}$ & $r_{e}^{3} f_{r \phi \phi \phi} / \mathrm{aJ} \AA^{-1}$ & 8.955 & 0.87 & & & 0.648 \\
\hline
\end{tabular}

${ }^{\mathrm{a}}$ From [58]. ${ }^{\mathrm{b}}$ From [138]. ${ }^{\mathrm{c}}$ From [109]. ${ }^{\mathrm{d}}$ From [57]. ${ }^{\mathrm{e}}$ From [139].

Following this approach, in [58] is described the vibrational spectrum of the most abundant isotopic species of water, $\mathrm{H}_{2}^{16} \mathrm{O}$. An energy fit was carried out for 72 experimental energies up to $23,000 \mathrm{~cm}^{-1}$, obtaining an rms deviation of $5.00 \mathrm{~cm}^{-1}$. The Hamiltonian (121) includes 17 force constants, the Morse parameters $\beta_{r}$ and $\beta_{\phi}$, plus the Child's parameters $\kappa_{s}$ and $\kappa_{b}$. Since the latter parameters are connected with the anharmonicity of the oscillators, they are estimated by considering the energy levels for one and two quanta. This procedure gives rise to the parameters $\kappa_{s}=47, \kappa_{b}=86$. The energy fit was carried out varying the linear parameters $z_{i}$ and the Morse frequencies $\widetilde{\omega}_{i}$, $i=r, \phi$, providing the set of force constants displayed in Table 3. In the first column of the table, the order needed in the expansions (48) and (49) in order to determine he corresponding force constants is indicated. All the force constants of order $1 / \kappa$ are not determined in the linear approximation (51).

A comparison with several description is presented. In general the force constants of second order follow the general trend of the previous calculations. For third- and quarticorder constants, however, large differences appear. This may be explained because of the difference in the calculations, in particular compared with the calculations of Jensen and the $a b$ initio results. This approach is quite simple, and the results may be compared with similar results, like the ones by Halonen and Carrington jr. [109] and Lemus et al. [57]. Indeed the force constants are similar.

Because of the differences in the more elaborated calculations, the reliability of the force constants was tested by predicting the energies for the isotopes $\mathrm{H}_{2}^{17} \mathrm{O}, \mathrm{H}_{2}^{18} \mathrm{O}, \mathrm{D}_{2}^{16} \mathrm{O}$, and $\mathrm{T}_{2}^{16} \mathrm{O}$, which can be found in Tables $\mathrm{x}, \mathrm{x}$, and $\mathrm{xxx}$ of [58].

Finally, we should mention that the Hamiltonian (121), which is impossible to obtain in more complex situations, is fundamental to interpret the different interactions derived from the use of the expansions (48) and (49). In practice, however, the symmetry-adapted expansion is not necessary.

\section{Application to Trifluoride of Boron}

In the description of vibrational excitations of molecules with high symmetry, the appearance of spurious states emerges in natural form when internal coordinates are used. The elimination of these extra degrees of freedom must be done from both the space and the Hamiltonian. Trifluoride of boron is a molecule that presents this feature, but it is also interesting because of its strong normal behavior. The description of this molecule in terms of normal modes is not a problem, the interesting situation appears when it is described in terms of a local mode model. As we know, the latter description cannot be carried out preserving the polyad. We show, however, that our algebraic approach 
allows this analysis to be done with the advantage of improving the description considerably.

Recently in a series of works the high-resolution infrared and Raman spectra of ${ }^{11} \mathrm{BF}_{3}$ from 400 to $4600 \mathrm{~cm}^{-1}$ were analyzed [116-120]. In these studies the analysis of the combination and overtone states led to the assignment of over 25,000 transitions. The number of pure vibrational energies, however, is reduced to around 25 band origins. The equilibrium configuration of the ${ }^{11} \mathrm{~B} \mathrm{~F}_{3}$ molecule is $D_{3 h}$ planar with structure parameter $r_{e}=1.3070 \AA$ [120]. It presents six vibrational degrees of freedom, three of them associated with the stretching modes $\left(A_{1}^{\prime} \oplus E^{\prime}\right)$, two of them corresponding to the bending modes $\left(E^{\prime}\right)$, and one out-ofplane mode $\left(A_{2}^{\prime \prime}\right)$. The harmonic approximation provides a complete basis in terms of normal coordinates. In the standard notation, this basis is labeled by $\left|\nu_{1} \nu_{2} v_{3}^{l_{3}} \nu_{4}^{l_{4}}\right\rangle$, where $v_{1}$ and $v_{3}$ correspond to the symmetrical and degenerate B-F stretching modes $A_{1}^{\prime}$ and $E^{\prime}$, respectively, while $\nu_{2}$ and $v_{4}$ correspond to the out-of-plane and degenerate $F-B-F$ bending modes $A_{2}^{\prime \prime}$ and $E^{\prime}$, respectively. Since the dipole operator spans the $A_{2}^{\prime \prime}$ and $E^{\prime}$ representations, only the $\nu_{1}$ vibrational mode is infrared inactive, the other three fundamentals are infrared active. Many of the combination and overtones states turn out to be infrared inactive as direct transitions from the ground states. However these states may be accessed as infrared-active hot bands transitions from thermally populated levels [118]. The fundamentals of ${ }^{11} \mathrm{BF}_{3}$ are $\left(\right.$ in $\left.\mathrm{cm}^{-1}\right)$

$$
\begin{array}{ll}
v_{1}=885.84, & v_{2}=691.21, \\
v_{3}=1453.96, & v_{4}=479.35,
\end{array}
$$

from which we identify the following allowed symmetry resonances:

$$
v_{1} \approx 2 v_{4}, \quad v_{3} \approx 3 v_{4}, \quad 2 v_{2} \approx 3 v_{4}
$$

The identification of these resonances permits to establish the polyad $P$, which in terms of integer numbers may be defined as

$$
P=4 v_{1}+3 v_{2}+6 v_{3}+2 v_{4}
$$

The structure of this polyad (209) reflects its strong normal behavior, which in turn is manifested in the fact that the interactions up to quadratic order will be dominated by diagonal contributions in a normal basis.

In Figure 1 of [66] the assignment of local displacement coordinates is displayed. The first set $\left(r_{i}, i=1,2,3\right)$ corresponds to the space of stretching oscillators $(s)$, the second set $\left(\theta_{i}, i=4,5,6\right)$ stands for the space of bending oscillators $(b)$, while the set $\left(\delta_{i}, \quad i=7,8,9\right)$ is associated with the out-of-plane angles $(\gamma)$ :

$$
\begin{aligned}
& q_{i}=\Delta r_{i}, \quad i=1,2,3, \\
& q_{j}=r_{e} \Delta \theta_{j}, \quad j=4,5,6, \\
& q_{k}=r_{e} \Delta \gamma_{k}, \quad k=7,8,9,
\end{aligned}
$$

where $r_{e}$ is the equilibrium distance B-F. We now proceed to obtain the symmetry-adapted coordinates $S_{\alpha}$, given by

$$
S_{x}^{\alpha}=\sum_{i} m_{i \alpha} q_{i}, \quad \alpha=1, \ldots, 9
$$

where $\alpha \equiv\{\Gamma, \mu\}$ stand for the irreps $\Gamma$ and $\mu$ of the $\mathscr{D}_{3 h}$ group and the subgroup $\mathcal{C}_{2}^{a}$, respectively, and $x$ refers to the subspace $(s, b, \gamma)$. Explicitly, we have for the stretching modes

$$
\begin{gathered}
S_{s}^{A_{1}^{\prime}}=\frac{1}{\sqrt{3}}\left(q_{1}+q_{2}+q_{3}\right), \\
S_{s}^{E^{\prime}, A}=\frac{1}{\sqrt{6}}\left(2 q_{1}-q_{2}-q_{3}\right), \\
S_{s}^{E^{\prime}, B}=\frac{1}{\sqrt{2}}\left(q_{2}-q_{3}\right)
\end{gathered}
$$

and for the bending coordinates

$$
\begin{gathered}
S_{b}^{A_{1}^{\prime}}=\frac{1}{\sqrt{3}}\left(q_{4}+q_{5}+q_{6}\right), \\
S_{b}^{E^{\prime}, A}=\frac{1}{\sqrt{6}}\left(q_{4}-2 q_{5}+q_{6}\right), \\
S_{b}^{E^{\prime}, B}=\frac{1}{\sqrt{2}}\left(q_{4}-q_{6}\right),
\end{gathered}
$$

while for the out-of-plane subspace

$$
\begin{gathered}
S_{\gamma}^{A_{2}^{\prime \prime}}=\frac{1}{\sqrt{3}}\left(q_{7}+q_{8}+q_{9}\right), \\
S_{\gamma}^{E^{\prime \prime}, A}=\frac{1}{\sqrt{6}}\left(-q_{8}+q_{9}\right), \\
S_{\gamma}^{E^{\prime \prime}, B}=\frac{1}{\sqrt{2}}\left(2 q_{7}-q_{8}-q_{9}\right) .
\end{gathered}
$$

The coordinates associated with the bending and out-ofplane coordinates in (125) are not independent. For the bending coordinates, the following redundant equation is given [62]:

$$
\sum_{i=4}^{6} q_{i}+\frac{1}{r_{e} \sqrt{3}}\left[\frac{1}{2} \sum_{i=4}^{6} q_{i}^{2}+\sum_{i>j=4}^{6}\left(q_{i} q_{j}\right)\right]+\cdots=0
$$

while for the out-of-plane coordinates

$$
\begin{gathered}
\left(q_{9}-q_{8}\right)-\frac{1}{r_{e} \sqrt{3}}\left(q_{4} q_{9}-q_{6} q_{8}\right)+\cdots=0 \\
\left(2 q_{7}-q_{8}-q_{9}\right)-\frac{1}{r_{e} \sqrt{3}}\left(2 q_{5} q_{7}-q_{6} q_{8}-q_{4} q_{9}\right)+\cdots=0 .
\end{gathered}
$$

These expressions, obtained through vectorial relations between the stretching vectors, allow the coordinates $\left\{S_{b}^{A_{1}^{\prime}}, S_{\gamma}^{E^{\prime \prime}, A}, S_{\gamma}^{E^{\prime \prime}, B}\right\}$ to be identified as redundant in the linear approximation. The transformation (127) is linear, but the transformations (128) and (129) are not linear in the sense that their inverse are nonlinear since the redundant 
equations (130) and (131) are to be satisfied. Indeed, in going up to the second order, the nonlinear transformations from the symmetrized coordinates to internal valence coordinates are found to be

$$
\begin{aligned}
& q_{4}=\frac{1}{\sqrt{6}} S_{b}^{E^{\prime}, A}+\frac{1}{\sqrt{2}} S_{b}^{E^{\prime}, B}, \\
& q_{5}=-\frac{2}{\sqrt{6}} S_{b}^{E^{\prime}, A}, \\
& q_{6}=\frac{1}{\sqrt{6}} S_{b}^{E^{\prime}, A}-\frac{1}{\sqrt{2}} S_{b}^{E^{\prime}, B}, \\
& q_{7}=\frac{1}{\sqrt{3}} S_{\gamma}^{A_{2}^{\prime \prime}}-\frac{1}{3 r_{e}} \sqrt{\frac{2}{3}} S_{\gamma}^{A_{2}^{\prime \prime}} S_{b}^{E^{\prime}, A}, \\
& q_{8}=\frac{1}{\sqrt{3}} S_{\gamma}^{A_{2}^{\prime \prime}}+\frac{1}{3 \sqrt{6} r_{e}} S_{\gamma}^{A_{2}^{\prime \prime}} S_{b}^{E^{\prime}, A}-\frac{1}{3 \sqrt{2} r_{e}} S_{\gamma}^{A_{2}^{\prime \prime}} S_{b}^{E^{\prime}, B}, \\
& q_{9}=\frac{1}{\sqrt{3}} S_{\gamma}^{A_{2}^{\prime \prime}}+\frac{1}{3 \sqrt{6} r_{e}} S_{\gamma}^{A_{2}^{\prime \prime}} S_{b}^{E^{\prime}, A}+\frac{1}{3 \sqrt{2} r_{e}} S_{\gamma}^{A_{2}^{\prime \prime}} S_{b}^{E^{\prime}, B} .
\end{aligned}
$$

Once the spurious coordinates are identified, the usual approach consist in rewriting the Hamiltonian (14) in terms of symmetry-adapted coordinates. According to the Lagrange's definition of classical momentum and using the chain rule, the local momenta are obtained from the symmetrized ones by the linear transformation

$$
\hat{p}_{i}=\sum_{\alpha} m_{i \alpha} \widehat{\mathcal{P}}_{\alpha},
$$

a relation that allows to express the kinetic energy in the form

$$
\widehat{T}=\frac{1}{2} \widetilde{P} g(\mathbf{q}) \mathcal{P},
$$

where $\mathcal{P}$ stands for the column vector of conjugate momenta associated with the symmetry coordinates. The transformed Wilson's matrix takes the form

$$
\mathcal{g}(\mathbf{q})=\mathbf{M}^{\dagger} \mathbf{G}(\mathbf{q}) \mathbf{M},
$$

where $\mathbf{M}=\left\|m_{i, \alpha}\right\|$. The Hamiltonian is obtained by adding to the kinetic energy the potential as a function of the new coordinates

$$
\hat{H}=\frac{1}{2} \widetilde{P} \mathcal{g} \mathcal{P}+V(\mathbf{S}) .
$$

A suitable Hamiltonian of practical interest is obtained by expanding the Wilson matrix as well as the potential in terms of physical symmetry-adapted coordinates up to quartic order. The resonances of higher order are later taken into account. Hence, the Hamiltonian is given in terms of a sum of contributions according to the different subspaces $(s$ stretching, $b$-bending, and $\gamma$-out-of-plane) plus additional interactions preserving the polyad

$$
\hat{H}=\hat{H}_{s}+\hat{H}_{b}+\hat{H}_{\gamma}+\hat{H}_{s b}+\hat{H}_{s \gamma}+\hat{H}_{b \gamma} .
$$

For the sake of simplification, we now introduce the variables

$$
\begin{array}{cc}
S_{s}^{A_{1}^{\prime}} \longrightarrow S_{1}, & S_{b}^{E^{\prime}, A} \longrightarrow S_{4 a}, \\
S_{s}^{E^{\prime}, A} \longrightarrow S_{3 a}, & S_{b}^{E^{\prime}, B} \longrightarrow S_{4 b}, \\
S_{s}^{E^{\prime}, B} \longrightarrow S_{3 b}, & S_{\gamma}^{A^{\prime \prime}} \longrightarrow S_{2},
\end{array}
$$

with similar notation for the momenta. The stretching Hamiltonian $\hat{H}_{s}$, for instance, takes the form

$$
\begin{aligned}
\hat{H}_{s}= & \frac{1}{2} g_{11}^{0} \hat{P}_{1}^{2}+\frac{1}{2} g_{3 a 3 a}^{0}\left(\hat{P}_{3 a}^{2}+\widehat{P}_{3 b}^{2}\right)+\frac{1}{2} F_{11} S_{1}^{2} \\
& +\frac{1}{2} F_{3 a 3 a}\left(S_{3 a}^{2}+S_{3 b}^{2}\right) \\
& +\frac{1}{4 !} F_{1111} S_{1}^{4}+\frac{6}{4 !} F_{113 a 3 a} S_{1}^{2}\left(S_{3 a}^{2}+S_{3 b}^{2}\right) \\
& +\frac{1}{4 !} F_{3 a 3 a 3 a 3 a}\left(S_{3 a}^{2}+S_{3 b}^{2}\right)^{2} .
\end{aligned}
$$

The factors $g_{\alpha \beta}^{0}$ stand for the matrix elements of the Wilson matrix evaluated at equilibrium. The next step in our approach consists in obtaining an algebraic representation of this Hamiltonian through the introduction of the bosonic operators associated with the symmetry-adapted coordinates

$$
\begin{array}{cc}
S_{1}=\frac{1}{2 \beta_{1}}\left(\hat{a}_{1}^{\dagger}+\hat{a}_{1}\right), & \hat{P}_{1}=i \hbar \beta_{1}\left(\hat{a}_{1}^{\dagger}-\hat{a}_{1}\right), \\
S_{3 a}=\frac{1}{2 \beta_{3}}\left(\hat{a}_{3 a}^{\dagger}+\hat{a}_{3 a}\right), & \hat{P}_{3 a}=i \hbar \beta_{3}\left(\hat{a}_{3 a}^{\dagger}-\hat{a}_{3 a}\right), \\
S_{3 b}=\frac{1}{2 \beta_{3}}\left(\hat{a}_{3 b}^{\dagger}+\hat{a}_{3 b}\right), & \hat{P}_{3 b}=i \hbar \beta_{3}\left(\hat{a}_{3 b}^{\dagger}-\hat{a}_{3 b}\right),
\end{array}
$$

where $\beta_{i}=(1 / 2 \hbar) \sqrt{F_{i i} / g_{i i}^{0}}$. The substitution of (140) into (139) leads to the algebraic representation

$$
\hat{H}_{s}=\hbar \Omega_{1} \hat{\nu}_{1}+\hbar \Omega_{3} \hat{\nu}_{3}+x_{11} \hat{v}_{1}^{2}+x_{33} \hat{\nu}_{3}^{2}+x_{13} \hat{\nu}_{1} \hat{\nu}_{3}+g_{s s} \hat{l}_{s}^{2} \text {, }
$$

where $\hat{v}_{i}$ are number operators defined by

$$
\hat{v}_{1}=\hat{a}_{1}^{\dagger} \hat{a}_{1}, \quad \hat{v}_{3}=\hat{a}_{3 a}^{\dagger} \hat{a}_{3 a}+\hat{a}_{3 b}^{\dagger} \hat{a}_{3 b}
$$

and $\hat{l}_{s}$ is the vibrational angular momentum given by

$$
l_{s}=-i \sqrt{2}\left[\hat{a}_{3}^{\dagger} \times a_{3}\right]^{A_{2}^{\prime}}
$$

where $\times$ stands for the coupling $E^{\prime} \times E^{\prime}$ to the irreps $A_{2}^{\prime}$. The spectroscopic parameters involved in (141) as well as in the rest of the contributions are function of the structure and force constants [62]. Following a similar approach for the rest of the contributions in (137) and adding the resonance terms of higher order, we obtain [62]

$$
\begin{aligned}
\hat{H}= & \sum_{i=1}^{4} \hbar \bar{\omega}_{i} \hat{\nu}_{i}+\sum_{i \leq j}^{4} x_{i j} \hat{\nu}_{i} \hat{\nu}_{j}+K_{34 / 34} \hat{N}_{34 / 34} \\
& +f_{1} \hat{F}_{1 / 44}+f_{2} \hat{F}_{3 / 444}+f_{3} \hat{F}_{22 / 444} \\
& +g_{s} \hat{l}_{s}^{2}+g_{b} \hat{l}_{b}^{2}+g_{s b} \hat{l}_{s} \cdot \hat{l}_{b}
\end{aligned}
$$


with similar definitions for the number operators and the vibrational angular momentum $\hat{l}_{b}$, and

$$
\begin{aligned}
\hat{F}_{3 / 444} & =\left[\hat{a}_{3}^{\dagger} \times\left[\left[\hat{a}_{4} \times \hat{a}_{4}\right]^{A_{1}^{\prime}} \times \hat{a}_{4}\right]^{E_{1}^{\prime}}\right]^{A_{1}^{\prime}}+H . c . \\
\hat{N}_{34 / 34} & =\left[\hat{a}_{3}^{\dagger} \times \hat{a}_{4}^{\dagger}\right]^{A_{1}^{\prime}} \times\left[\hat{a}_{3} \times \hat{a}_{4}\right]^{A_{1}^{\prime}}, \\
\hat{F}_{1 / 44} & =\hat{a}_{1}^{\dagger} \times\left[\hat{a}_{4} \times \hat{a}_{4}\right]^{A_{1}^{\prime}}+H . c ., \\
\hat{F}_{22 / 444} & =\hat{a}_{2}^{\dagger} \times \hat{a}_{2}^{\dagger} \times\left[\left[a_{4} \times \hat{a}_{4}\right]^{E_{1}^{\prime}} \times \hat{a}_{4}\right]^{A_{1}^{\prime}}+\text { H.c. }
\end{aligned}
$$

The Hamiltonian (144) may be diagonalized in a harmonic oscillator basis. Instead we will introduce, in similar form to (67), the following canonical transformation in terms of local bosons (after the substitution $\hat{c}_{i}^{\dagger} \rightarrow \hat{a}_{i}^{\dagger}$ ):

$$
\hat{a}_{x}^{\Gamma \mu \dagger}=\sum_{i} m_{i ; \Gamma, \mu, x} \hat{a}_{i}^{\dagger}
$$

where $x$ stands for the subspace and the matrix $\mathbf{M}=$ $\left\|m_{i ; \Gamma, \gamma, x}\right\|$ corresponds to the coefficients in the projections (127), (128), and (129). We now introduce our proposal of anharmonization

$$
\hat{a}_{i}^{\dagger} \longrightarrow \hat{b}_{i}^{\dagger}, \quad a_{i} \longrightarrow \hat{b}_{i}
$$

where $\hat{b}_{i}^{\dagger}\left(\hat{b}_{i}\right)$ are identified with creation and annihilation operators for the Morse functions for the stretching and bending spaces and Pöschl-Teller functions for the out-ofplane subspace. This step is crucial in our method since significant improvements are implied, without a straightforward analog in configuration space. With the proposal (147), we interpret the new Hamiltonian as an algebraic local representation in terms of operators defined in a space of Morse functions for the stretches and bending oscillators and Pöschl-Teller functions for the out-of-plane modes.

Hence, in the framework of the anharmonization procedure, the Hamiltonian that we propose takes the isomorphic form (144)

$$
\begin{aligned}
\widehat{\mathscr{H}}= & \sum_{i=1}^{4} \hbar \bar{\omega}_{i} \hat{\bar{\nu}}_{i}+\sum_{i \geq j} x_{i j} \frac{1}{2}\left(\hat{\bar{v}}_{i} \hat{\bar{\nu}}_{j}+\hat{\bar{v}}_{j} \hat{\bar{v}}_{i}\right)+K_{34 / 34} \hat{\bar{N}}_{34 / 34} \\
& +f_{1} \hat{\bar{F}}_{1 / 44}+f_{2} \hat{\bar{F}}_{3 / 444}+f_{3} \hat{\bar{F}}_{22 / 444} \\
& +g_{s} \hat{\bar{l}}_{s}^{2}+g_{b} \hat{\bar{l}}_{b}^{2}+g_{s b} \hat{\bar{l}}_{s} \cdot \hat{\bar{l}}_{b}
\end{aligned}
$$

but the new operators defined with bars are given by

$$
\begin{gathered}
\hat{\bar{\nu}}_{1}=\left[\hat{T}_{s}^{\dagger A_{1}^{\prime}} \times \widehat{T}_{s}^{A_{1}^{\prime}}\right]^{A_{1}^{\prime}}, \\
\hat{\bar{v}}_{2}=\left[\hat{T}_{\gamma}^{\dagger A_{1}^{\prime \prime}} \times \widehat{T}_{\gamma}^{A_{1}^{\prime \prime}}\right]^{A_{1}^{\prime}}, \\
\hat{\bar{\nu}}_{3}=\sqrt{2}\left[\hat{T}_{s}^{\dagger E^{\prime}} \times \widehat{T}_{s}^{E^{\prime}}\right]^{A_{1}^{\prime}}, \\
\hat{\bar{\nu}}_{4}=\sqrt{2}\left[\hat{T}_{b}^{\dagger E^{\prime}} \times \widehat{T}_{b}^{E^{\prime}}\right]^{A_{1}^{\prime}},
\end{gathered}
$$

while for the resonances

$$
\begin{aligned}
& \widehat{F}_{1 / 44}=\left[\hat{T}_{s}^{\dagger A_{1}^{\prime}} \times\left[\hat{T}_{b}^{E^{\prime}} \times \widehat{T}_{b}^{E^{\prime}}\right]^{A_{1}^{\prime}}\right]^{A_{1}^{\prime}}+\text { H.c. }, \\
& \widehat{N}_{34 / 34}=\left[\left[\widehat{T}_{s}^{+E^{\prime}} \times \widehat{T}_{b}^{\dagger E^{\prime}}\right]^{A_{1}^{\prime}} \times\left[\hat{T}_{s}^{E^{\prime}} \times \widehat{T}_{b}^{E^{\prime}}\right]^{A_{1}^{\prime}}\right]^{A_{1}^{\prime}}+\text { H.c. }, \\
& \widehat{F}_{3 / 444}=\left[\widehat{T}_{s}^{+E^{\prime}} \times\left[\left[\hat{T}_{b}^{E^{\prime}} \times \widehat{T}_{b}^{E^{\prime}}\right]^{A_{1}^{\prime}} \times \widehat{T}_{b}^{E^{\prime}}\right]^{E^{\prime}}\right]^{A_{1}^{\prime}}+\text { H.c. }, \\
& \widehat{F}_{22 / 444}=\hat{T}_{\gamma}^{\dagger A_{2}^{\prime \prime}} \times \widehat{T}_{\gamma}^{\dagger A_{2}^{\prime \prime}} \times\left[\left[\hat{T}_{b}^{E^{\prime}} \times \widehat{T}_{b}^{E^{\prime}}\right]^{E^{\prime}} \times \widehat{T}_{b}^{E^{\prime}}\right]^{A_{1}^{\prime}}+\text { H.c. },
\end{aligned}
$$

with tensors given by

$$
\widehat{T}_{x}^{\dagger \Gamma, \mu}=\sum_{i} m_{i ; x \Gamma \mu} \hat{b}_{i}^{\dagger}
$$

where $x$ stands for the space. The coefficients are the one obtained in the symmetry-adapted coordinates, in accordance with (126), with the isomorphism

$$
\begin{aligned}
\hat{a}_{1}^{\dagger} \longleftrightarrow \hat{T}_{s}^{\dagger A_{1}^{\prime}}, & \hat{a}_{2}^{\dagger} \longleftrightarrow \hat{T}_{\gamma}^{\dagger A_{1}^{\prime \prime},} \\
\hat{a}_{3 a}^{\dagger} \longleftrightarrow \hat{T}_{s}^{\dagger E^{\prime}, A}, & \hat{a}_{3 b}^{\dagger} \longleftrightarrow \widehat{T}_{s}^{\dagger E^{\prime}, B}, \\
\hat{a}_{4 a}^{\dagger} \longleftrightarrow \hat{T}_{b}^{\dagger E^{\prime}, A}, & \hat{a}_{4 b}^{\dagger} \longleftrightarrow \hat{T}_{b}^{\dagger E^{\prime}, B},
\end{aligned}
$$

and similar correspondence for the adjoint operators. The Hamiltonian (148) deserves the following comment. Once the anharmonization procedure is applied, the number operators involving the same space stop commuting, which explains the form of the symmetrized anharmonic contribution. On the other hand, we may intend to diagonalize this Hamiltonian in the local basis given in terms of the direct product (in configuration space)

$$
\Psi_{\mathbf{v}}(\mathbf{y}, \mathbf{u})=\prod_{i=1}^{6} \Psi_{v_{i}}^{M}\left(y_{i}\right) \prod_{j=7}^{9} \Psi_{v_{j}}^{P T}\left(u_{j}\right),
$$

where $\Psi_{v_{i}}^{M}\left(y_{i}\right)$ and $\Psi_{v_{j}}^{P T}\left(y_{j}\right)$ are Morse and PT functions, respectively. However, this is not possible due to the existence of spurious modes. These redundancies should be eliminated from the basis. To achieve this task we follow an approach based on the construction of a basis obtained from the diagonalization of the representation of a complete set of commuting operators in the local basis, which leads to a symmetry-adapted basis isomorphic to the normal basis $[62,89,121]$.

The available vibrational experimental data is not as abundant as we could wish. We have considered the 25 experimental energies available in [120]. The Hamiltonian (148) involves 21 interactions, but not all the parameters are able to be determined with the available experimental data. On the other hand, the diagonalization of the Hamiltonian (148) implies fixing the parameters $\kappa_{s}, \kappa_{b}$, and $\kappa_{\gamma}$ associated with the depth of the local Morse and PT potentials. The approach we have followed to estimate the parameters 
TABLE 4: Experimental and calculated energies, given as differences of energy, for ${ }^{11} \mathrm{BF}_{3}$. Fit 1 is obtained by diagonalizing the Hamiltonian (148), while Fit 2 corresponds to the equivalent description in the harmonic limit. In the last row the rms is displayed.

\begin{tabular}{|c|c|c|c|c|c|}
\hline \multirow[t]{2}{*}{ Polyad } & \multicolumn{2}{|c|}{ State } & \multirow{2}{*}{$\begin{array}{c}\text { Exp. [120] } \\
\text { Symmetry } A_{1}^{\prime}\end{array}$} & \multirow[t]{2}{*}{ Fit 1} & \multirow[t]{2}{*}{ Fit 2} \\
\hline & & & & & \\
\hline 4 & $1_{1}$ & & 885.84 & 0.4 & 2.4 \\
\hline 4 & $4_{2}$ & & 959.31 & 0.9 & 0.2 \\
\hline 6 & $22_{2}$ & & 1384.96 & 0. & 0.3 \\
\hline \multirow[t]{2}{*}{8} & $3_{1}$ & $4_{1}$ & 1929.68 & 0 & 0.0 \\
\hline & & & Symmetry $E^{\prime}$ & & \\
\hline 2 & $4_{1}$ & & 479.35 & -0.2 & -3 \\
\hline 4 & $4_{2}$ & & 959.69 & -0.7 & 0.6 \\
\hline 6 & $1_{1}$ & $4_{1}$ & 1361.32 & 0.2 & -1 . \\
\hline 6 & $3_{1}$ & & 1453.96 & 0. & -5.4 \\
\hline 8 & $1_{1}$ & $4_{2}$ & 1837.73 & -0.2 & 2.1 \\
\hline 8 & $22_{2}$ & $4_{1}$ & 1866.12 & 0. & 0.5 \\
\hline 8 & $3_{1}$ & $4_{1}$ & 1931.92 & 0. & 0 \\
\hline 10 & $1_{2}$ & $4_{1}$ & 2240.94 & 0.1 & 0.3 \\
\hline 10 & $1_{1}$ & $3_{1}$ & 2336.20 & -0.7 & -5.1 \\
\hline 12 & $1_{1}$ & $3_{1} \quad 4_{1}$ & 2810.70 & -0.3 & 0.3 \\
\hline 12 & $3_{2}$ & & 2905.36 & 0.5 & 7.8 \\
\hline 14 & $1_{3}$ & $4_{1}$ & 3118.20 & -0.1 & 1. \\
\hline 14 & $1_{2}$ & $3_{1}$ & 3216.32 & -0.1 & -5.3 \\
\hline 16 & $1_{2}$ & $3_{1} \quad 4_{1}$ & 3687.14 & 0.4 & -0.1 \\
\hline 16 & $1_{1}$ & $3_{2}$ & 3783.84 & 0.3 & 5.7 \\
\hline \multirow[t]{2}{*}{18} & $3_{3}$ & & 4310.26 & -0.3 & -4.5 \\
\hline & & & Symmetry $A_{2}^{\prime \prime}$ & & \\
\hline 3 & $2{ }_{1}$ & & 691.21 & 0 & 0.4 \\
\hline 7 & $1_{1}$ & $2{ }_{1}$ & 1573.73 & 0 & -0.2 \\
\hline \multirow[t]{2}{*}{9} & $2{ }_{3}$ & & 2081.12 & 0. & -0.3 \\
\hline & & & Symmetry $E^{\prime \prime}$ & & \\
\hline 5 & $2{ }_{1}$ & $4_{1}$ & 1171.49 & 0. & -1 \\
\hline 9 & $2_{1}$ & $3_{1}$ & 2139.68 & 0 & 0 \\
\hline $\mathrm{rms}$ & & & & 0.52 & 4.69 \\
\hline
\end{tabular}

consists in taking the best values arisen from a series of fits involving the 25 experimental energies considering only the harmonic $\omega_{i}$ and anharmonic contributions $x_{i j}$ (14 parameters). The best values were found to be $\kappa_{s}=50, \kappa_{b}=170$ and $\kappa_{\gamma}=180$. The spectroscopic parameters are thereafter optimized by a least square method for fixed $\kappa_{s}, \kappa_{b}$, and $\kappa_{\gamma}$. In Table 4 we present the 25 experimental and the theoretical energies provided by two fits. Fit 1 refers to the case where the anharmonicities $x_{i j}$ as well as the resonance $\widehat{N}_{34 / 34}$ are taken into account. Adding additional interactions makes the calculation unstable [62]. The deviation obtained was rms $=0.52 \mathrm{~cm}^{-1}$. A criterion to evaluate our approach consists in carrying out a comparison with the equivalent description in the harmonic limit (an equivalent description in configuration space). This test is displayed in the column labeled as Fit 2, providing a square root deviation of $\mathrm{rms}=$ $4.69 \mathrm{~cm}^{-1}$. A comparison of our description with this fit
TABLE 5: Force constants derived from the spectroscopic parameters provided by Fit 3 .

\begin{tabular}{lcc}
\hline Parameter & This work [62] & Ab initio [140] \\
\hline$F_{11}\left(\mathrm{aJ} \AA^{-2}\right)$ & 9.043 & 9.052 \\
$F_{22}\left(\mathrm{aJ} \AA^{-2}\right)$ & 0.0979 & \\
$F_{33}\left(\mathrm{aJ} \AA^{-2}\right)$ & 7.018 & 7.018 \\
$F_{44}\left(\mathrm{aJ} \AA^{-2}\right)$ & 0.2439 & 0.52 \\
\hline$F_{1111}\left(\mathrm{aJ} \AA^{-4}\right)$ & 499.46 & 100.359 \\
$F_{2222}\left(\mathrm{aJ} \AA^{-4}\right)$ & 0.0428 & \\
$F_{3 a 3 a 3 a 3 a}\left(\mathrm{aJ} \AA^{-4}\right)$ & 168.24 & 127.364 \\
$F_{4 a 4 a 4 a 4 a}\left(\mathrm{aJ} \AA^{-4}\right)$ & 0.2925 & 1.090 \\
\hline$F_{1122}\left(\mathrm{aJ} \AA^{-4}\right)$ & -0.9345 & 89.376 \\
$F_{113 a 3 a}\left(\mathrm{aJ} \AA^{-4}\right)$ & 317.65 & 2.225 \\
$F_{114 a 4 a}\left(\mathrm{aJ} \AA^{-4}\right)$ & -0.0259 & \\
$F_{223 a 3 a}\left(\mathrm{aJ} \AA^{-4}\right)$ & -0.8432 & -0.305 \\
$F_{224 a 4 a}\left(\mathrm{aJ} \AA^{-4}\right)$ & -0.0023 & 1.820 \\
$F_{3 a 3 a 4 b 4 b}\left(\mathrm{aJ} \AA^{-4}\right)$ & -0.748 & \\
\hline$F_{3 a 4 a 4 a 4 a}\left(\mathrm{aJ} \AA^{-4}\right)$ & -1.335 &
\end{tabular}

allows us to conclude that the local approach based on anharmonic interacting oscillators is significatively better. The obtained force constants are displayed in Table 5.

To finish this section we will include some comments concerned with the approach to consider polyad breaking effects. The problem of polyad breaking is an old subject that has attracted the attention since many years ago. It has been analyzed using different techniques, with their associated features and limitations. Full variational methods with exact kinetic energy operators have been successful to obtain a good description of fairly high vibrational excitations, but unfortunately these methods are computationally very demanding and they cannot in practice be extended to large or even medium-sized molecules [2]. Recursive techniques based on Lanczos method have been developed, but it seems that they are not well suited to the study of overtone line shapes [106]. Alternative approaches based on the semiclassical description of molecular vibrations have been also developed, although difficulties arise at higher energies, where chaotic behavior is dominant [106]. A powerful approach to take into account the effect of polyad mixing is based on the canonical Van Vleck perturbation theory $[4,9]$. The basic idea consists in transforming the Hamiltonian, expressed as an expansion in perturbative contributions $H^{(n)}$, to a new representation via a series of unitary transformations where the effect of the interactions connecting states of different polyads is taken into account inside the polyad blocks. Because of the perturbative nature of this approach, a crucial ingredient consists in providing the order of the different contributions to the Hamiltonian. Recently a general and systematic approach to deal with the problem of polyad breaking when Morse potentials are used to describe the internal degrees of freedom of molecular systems has been established [101]. 


\section{0. $U(v+1)$ Algebraic Model for Vibrations}

The $S U(2)$ model obtained by adding an extra boson to the one-dimensional harmonic oscillator can be extended in two directions. If we were interested in describing simultaneously both vibration and rotations of diatomic molecules, the appropriate extension would lead to the $U(4)$ model [23]. Another possibility, however, consists in extending the model by including several local vibrational degrees of freedom of the same subspace. This effort gives rise to the unitary approach $U(\nu+1)$ proposed by Michelot and Moret-bailly [44]. In this section we will discuss this approach, presenting a proposal to establish the connection with configuration space, and in this way being able to estimate the PES. Results for pyramidal molecules are presented [63-66].

We start considering $v$ equivalent harmonic oscillators. Associated with the $i$ th oscillator, we have bosonic creation $\hat{a}_{i}^{\dagger}$ and annihilation $\hat{a}_{i}$ operators. In the framework of the $U(\nu+1)$ approach an additional $\hat{s}$ boson is added with the constraint that the total number of bosons $\hat{N}$ is constant. The generators of the group $U(\nu+1)$ are given by

$$
\hat{C}_{i}^{j}=\hat{c}_{i}^{\dagger} \widehat{c}_{j}, \quad \hat{c}_{i}=\hat{a}_{i}, \quad i=1, \ldots, v, \quad \hat{c}_{\nu+1}=\hat{s}
$$

with commutation relations

$$
\left[\hat{C}_{i}^{j}, \hat{C}_{p}^{q}\right]=\hat{C}_{i}^{q} \delta_{p, j}-\hat{C}_{p}^{j} \delta_{q, i},
$$

where the extra boson has been included. In this model the state vectors associated with a local mode description of a molecular system consist of a set of $v+1$ independent harmonic oscillators. Explicitly this basis is given by

$$
\left|[N] ; n_{1}, n_{2}, \ldots, n_{v}, n_{s}\right\rangle=\frac{1}{\sqrt{n_{s} ! \prod_{j}^{v} n_{j} !}}\left(\hat{s}^{\dagger}\right)^{n_{s}} \prod_{i}^{v}\left(\hat{a}_{i}^{\dagger}\right)^{n_{i}}|0\rangle
$$

which are characterized by the total number of quanta $N$, whose corresponding operator is given by

$$
\hat{N}=\hat{n}+\hat{n}_{s} \text {, }
$$

with

$$
\hat{n}=\sum_{i=1}^{v} \hat{a}_{i}^{\dagger} \hat{a}_{i}, \quad \hat{n}_{s}=\hat{s}^{\dagger} \hat{s} .
$$

The total number of bosons fixes the totally symmetric representation $[N]$ of the $U(\nu+1)$ group, and because of the relation (157) we can rewrite the kets (156) in the form

$$
\left|[N], n ; n_{1}, n_{2}, \ldots, n_{\nu}\right\rangle \equiv\left|[N] ; n_{1}, n_{2}, \ldots, n_{\nu}, n_{s}\right\rangle .
$$

The addition of the $\hat{s}$ boson together with the fact that the representation $[N]$ is fixed makes the unitary group $U(\nu+1)$ a dynamical group for the set of $\nu$ oscillators.

From the generators (154) of the unitary group $U(\nu+1)$ we identify $\nu$-su(2) subalgebras with generators

$$
\hat{J}_{i,+}=\hat{s} \hat{a}_{i}^{\dagger}, \quad \hat{J}_{i,-}=\hat{s}^{\dagger} \hat{a}_{i}, \quad \hat{J}_{i, 0}=-\frac{1}{2}\left(\hat{s}^{\dagger} \hat{s}-\hat{a}_{i}^{\dagger} \hat{a}_{i}\right),
$$

with the usual angular momentum commutation relations

$$
\left[\widehat{J_{i,+}}, \hat{J_{i,-}}\right]=2 \widehat{J_{i, 0}}, \quad\left[\hat{J_{i, 0}}, \widehat{J_{i, \pm}}\right]= \pm \widehat{J_{i, \pm}} \text {. }
$$

We now introduce the normalized operators

$$
\hat{b}_{i}^{\dagger} \equiv \frac{\hat{J}_{i,+}}{\sqrt{N}}, \quad \hat{b}_{i} \equiv \frac{\hat{J}_{i,-}}{\sqrt{N}}
$$

which satisfy the commutation relations

$$
\left[\hat{b}_{i}, \hat{b}_{j}^{\dagger}\right]=\delta_{i j}-\frac{1}{N}\left[\hat{n} \delta_{i j}+\hat{a}_{j}^{\dagger} \hat{a}_{i}\right], \quad\left[\hat{b}_{i}^{\dagger}, \hat{b}_{j}^{\dagger}\right]=\left[\hat{b}_{i}, \hat{b}_{j}\right]=0
$$

In particular

$$
\left[\hat{b}_{i}, \hat{b}_{i}^{\dagger}\right]=1-\frac{1}{N}\left[\hat{n}+\hat{n}_{i}\right]=-\frac{2}{N} \widehat{J}_{i, 0}
$$

The action of these operators over the kets (159) is the following:

$$
\begin{aligned}
\hat{b}_{i}^{\dagger}\left|[N], n ; n_{1}, n_{2}, \ldots, n_{\nu}\right\rangle= & \sqrt{\left(n_{i}+1\right)\left(1-\frac{n}{N}\right)} \\
& \times\left|[N], n+1 ; n_{1}, \ldots, n_{i}+1, \ldots, n_{\nu}\right\rangle, \\
\hat{b}_{i}\left|[N], n ; n_{1}, n_{2}, \ldots, n_{\nu}\right\rangle= & \sqrt{n_{i}\left(1-\frac{n-1}{N}\right)} \\
& \times\left|[N], n-1 ; n_{1}, \ldots, n_{i}-1, \ldots, n_{\nu}\right\rangle,
\end{aligned}
$$

while for the operators $\hat{a}_{i}^{\dagger}\left(\hat{a}_{i}\right)$

$$
\begin{aligned}
& \hat{a}_{i}^{\dagger}\left|[N], n ; n_{1}, n_{2}, \ldots, n_{\nu}\right\rangle \\
& \quad=\sqrt{\left(n_{i}+1\right)}\left|[N], n+1 ; n_{1}, \ldots, n_{i}+1, \ldots, n_{\nu}\right\rangle, \\
& \hat{a}_{i}\left|[N], n ; n_{1}, n_{2}, \ldots, n_{\nu}\right\rangle \\
& =\sqrt{n_{i}}\left|[N], n-1 ; n_{1}, \ldots, n_{i}-1, \ldots, n_{\nu}\right\rangle .
\end{aligned}
$$

From these results, it is clear that in the harmonic limit $N \rightarrow$ $\infty$ the operators $\left\{\hat{b}_{i}^{\dagger}, \hat{b}_{i}\right\}$ go to the bosonic operators $\left\{\hat{a}_{i}^{\dagger}, \hat{a}_{i}\right\}$, as long as $n$ remains finite. This is indeed the case when in the calculations the polyad number is used. In fact, from (165) and (166) we obtain

$$
\lim _{N \rightarrow \infty} \hat{b}_{i}^{\dagger}=\hat{a}_{i}^{\dagger}, \quad \lim _{N \rightarrow \infty} \hat{b}_{i}=\hat{a}_{i},
$$

and consequently

$$
\lim _{N \rightarrow \infty}\left[\hat{b}_{i}, \hat{b}_{j}^{\dagger}\right]=\delta_{i j}
$$

When $\alpha$ sets of equivalent oscillators are present in the vibrational description, a $u(\nu+1)$ algebra is introduced for each equivalent set, so that the dynamical group is given by the direct product

$$
U_{1}\left(\nu_{1}+1\right) \times U_{2}\left(\nu_{2}+1\right) \times \cdots \times U_{\alpha}\left(\nu_{\alpha}+1\right) .
$$


In this case a total number of bosons $N_{i}$ is associated with each space, which in turn is related to the dissociation limit of the corresponding internal coordinates [44].

The model summarized above permits to obtain a spectroscopic description based on energy and intensity fittings. However, extracting force constants is crucial to establish the connection between the generators of the dynamical algebra and the local coordinates and momenta, at least in approximate form. We propose the following approximation for the local coordinates $q_{i}$ and momenta $\hat{p}_{i}$ :

$$
\begin{gathered}
q_{i} \simeq \sqrt{\frac{\hbar}{2 \omega \mu}}\left(\hat{d}_{i}^{\dagger}+\hat{d}_{i}\right), \\
\hat{p}_{i} \simeq \frac{i}{2} \sqrt{2 \hbar \omega \mu}\left(\hat{d}_{i}^{\dagger}-\hat{d}_{i}\right),
\end{gathered}
$$

where $\hat{d}^{\dagger}(\hat{d})$ may be identified either with $\hat{a}^{\dagger}(\hat{a})$ or $\hat{b}^{\dagger}(\hat{b})$. The connection (170) represents just an approximation, not only because we are proposing a linear expansion in terms of the operators (162) but also because the commutation relations $\left[q_{i}, \hat{p}_{j}\right]=i \hbar \delta_{i j}$ are only nearly satisfied. We know however that these relations are exact in the harmonic limit, a remarkable fact that suggests the approximate connection (170) with configuration space.

In order to decide the appropriate correspondence $\hat{a}^{\dagger}(\hat{a})$ or $\hat{b}^{\dagger}(\hat{b})$, we will first analyze the case where all operators belong to the same set of equivalent oscillators $\alpha$. In this case when the operators $\hat{d}^{\dagger}(\hat{d})$ are associated with $\hat{a}^{\dagger}(\hat{a})$, the usual commutation relation for the coordinates and momenta are satisfied. In contrast, using the operators $\hat{b}^{\dagger}(\hat{b})$ we obtain

$$
\left[q_{i}, q_{j}\right]=\frac{\hbar}{2 \omega \mu} \frac{1}{N}\left[\hat{a}_{j}^{\dagger} \hat{a}_{i}-\hat{a}_{i}^{\dagger} \hat{a}_{j}^{\dagger}\right], \quad i, j \in \alpha,
$$

while for the coordinates and momenta

$$
\left[q_{i}, \hat{p}_{j}\right]=i \hbar \delta_{i j}-\frac{i \hbar}{2 N}\left[2 \hat{n}_{i j}+\hat{a}_{j} \hat{a}_{i}^{\dagger}+\hat{a}_{i} \hat{a}_{j}^{\dagger}\right], \quad i, j \in \alpha .
$$

We may thus conclude that the correspondence $\hat{d}^{\dagger}(\hat{d}) \rightarrow$ $\hat{a}^{\dagger}(\hat{a})$ must be considered for contributions of the Hamiltonian that involve one set of equivalent coordinates, otherwise the results of the fit would depend on the order of the local coordinates and momenta in the expansion. However, we can bypass this problem by proposing a symmetrization when we select the operators $\hat{b}^{\dagger}(\hat{b})$ as we will discuss.

This approach allows the Born-Oppenheimer potential surfaces to be obtained. As an example the spectroscopic description of Arsine will be presented, where the force constants are estimated as well as transition intensities. The equilibrium structure of the arsine molecule is pyramidal with structure parameters $r_{e}=1.51106 \AA$, and $\angle H A s H=$ $92.069^{\circ}$ [122]. The point symmetry group is $\mathcal{C}_{3 v}$. This molecule has six degrees of freedom, three of them associated with the stretching modes $\left(A_{1} \oplus E\right)$ and three bending modes $\left(A_{1} \oplus E\right)$. In the standard notation the harmonic basis is labeled by

$$
\left|v_{1} v_{2} v_{3}^{l_{3}} v_{4}^{l_{4}}\right\rangle
$$

where $v_{1}$ and $v_{3}$ correspond to the symmetrical and degenerate $A s-H$ stretching modes $A_{1}$ and $E$, respectively, while $\nu_{2}$ and $\nu_{4}$ correspond to the symmetrical and degenerate $H$-As$H$ bending modes $A_{1}$ and $E$, respectively. $l_{3}$ and $l_{4}$ correspond to the projection of the angular momenta associated with the corresponding degenerate modes. Since here we will restrict ourselves to the study of the stretching degrees of freedom, the normal states will be labeled as $\left|\nu_{1} v_{3}^{l_{3}}\right\rangle$ or $\left|1_{\nu_{1}}, 3 \nu_{3}\right\rangle$. We thus start by expanding both the Wilson matrix as well as the potential in terms of the displacement local coordinates. Since the elements $g_{r r}$ and $g_{r r^{\prime}}$ of the $\mathbf{G}(\mathbf{q})$ matrix does not depend on the stretching variables, the expansion reduces to $g_{i j}(\mathbf{q})=g_{i j}^{0}$. The Hamiltonian takes thus the form

$$
\begin{aligned}
\hat{H}= & \frac{1}{2} g_{r r}^{o} \sum_{i=1}^{3} \hat{p}_{i}^{2}+g_{r r^{\prime}}^{o} \sum_{i>j=1}^{3} \hat{p}_{i} \hat{p}_{j}+\frac{1}{2} f_{r r} \sum_{i=1}^{3} q_{i}^{2}+f_{r r^{\prime}} \sum_{i>j=1}^{3} q_{i} q_{j} \\
& +\frac{1}{4 !} f_{r r r r} \sum_{i=1}^{3} q_{i}^{4}, \\
& +\frac{4}{4 !} f_{r r r r^{\prime}}\left[q_{1}^{3}\left(q_{2}+q_{3}\right)+q_{2}^{3}\left(q_{1}+q_{3}\right)+q_{3}^{3}\left(q_{1}+q_{2}\right)\right] \\
& +\frac{3}{4 !} f_{r r r^{\prime} r^{\prime}}\left[q_{1}^{2}\left(q_{2}^{2}+q_{3}^{2}\right)+q_{2}^{2}\left(q_{3}^{2}+q_{1}^{2}\right)+q_{3}^{2}\left(q_{1}^{2}+q_{2}^{2}\right)\right] \\
& +\frac{12}{4 !} f_{r r r^{\prime} r^{\prime \prime}}\left[q_{1}^{2} q_{2} q_{3}+q_{2}^{2} q_{1} q_{3}+q_{3}^{2} q_{1} q_{3}\right]
\end{aligned}
$$

where only terms leading to preservation of the polyad

$$
P=n_{1}+n_{2}+n_{3}
$$

have been considered.

The next step consists in obtaining the algebraic representation of the Hamiltonian. In principle we could carry out the substitution of (170) with the identification $\hat{d}^{\dagger}(\hat{d})$ with $\hat{b}^{\dagger}(\hat{b})$, but this realization would not be unique. Consequently we propose to substitute each term by its symmetric form. For the quadratic terms we thus carry out the substitutions

$$
\hat{p}_{i} \hat{p}_{j} \longrightarrow \frac{1}{2}\left(\hat{p}_{i} \hat{p}_{j}+\hat{p}_{j} \hat{p}_{i}\right), \quad q_{i} q_{j} \longrightarrow \frac{1}{2}\left(q_{i} q_{j}+q_{j} q_{i}\right)
$$

while for the quartic terms appearing in the potential

$$
\begin{aligned}
q_{1}^{3}\left(q_{2}+q_{3}\right) & \longrightarrow \frac{1}{2}\left[q_{1}^{3}\left(q_{2}+q_{3}\right)+\left(q_{2}+q_{3}\right) q_{1}^{3}\right] \\
q_{1}^{2}\left(q_{2}^{2}+q_{3}^{2}\right) \longrightarrow & \frac{1}{2}\left[q_{1}^{3}\left(q_{2}^{2}+q_{3}^{2}\right)+\left(q_{2}^{2}+q_{3}^{2}\right) q_{1}^{3}\right] \\
q_{1}^{2} q_{2} q_{3} \longrightarrow & \frac{1}{6}\left[q_{1}^{2}\left(q_{2} q_{3}+q_{3} q_{2}\right)+\left(q_{2} q_{3}+q_{3} q_{2}\right) q_{1}^{2}\right. \\
& \left.+q_{3} q_{1}^{2} q_{2}+q_{2} q_{1}^{2} q_{3}\right] .
\end{aligned}
$$

We now reconsider the arguments to identify $\hat{d}^{\dagger}(\hat{d})$ either with $\hat{b}^{\dagger}(\hat{b})$ or $\hat{a}^{\dagger}(\hat{a})$. We have found that the best selection 
corresponds to the substitution $\hat{d}^{\dagger}(\hat{d}) \rightarrow a^{\dagger}(\hat{a})$ in the quadratic terms as well as in the higher-order terms involving number operators, while $\hat{d}^{\dagger}(\hat{d}) \rightarrow b^{\dagger}(\hat{b})$ into the rest of the terms. The Hamiltonian takes thus the form in wave numbers $\left(\mathrm{cm}^{-1}\right)$

$$
\frac{\hat{H}}{h c}=\widetilde{\omega}_{e} \hat{n}+\lambda \sum_{i \neq j}^{3} \hat{a}_{i}^{\dagger} \widehat{a}_{j}+\alpha_{1} \hat{I}_{1}+\sum_{i=2}^{4} \alpha_{j}\left(\sum_{p} \hat{\mathcal{O}}_{p} \hat{I}_{i}\right),
$$

where the local interactions $\widehat{I}_{i}$ are

$$
\begin{aligned}
\hat{I}_{1}= & \sum_{i=1}^{3}\left(\hat{a}_{i}^{\dagger} \hat{a}_{i}\right)^{2}=\sum_{i=1}^{3} \hat{n}_{i}^{2}, \\
\hat{I}_{2}= & \hat{b}_{1}^{\dagger 2} \hat{b}_{1}\left(\hat{b}_{2}+\hat{b}_{3}\right)+\hat{b}_{1}^{2} \hat{b}_{1}^{\dagger}\left(\hat{b}_{2}^{\dagger}+\hat{b}_{3}^{\dagger}\right)+\left(\hat{b}_{1}^{\dagger} \hat{b}_{1}+\hat{b}_{1} \hat{b}_{1}^{\dagger}\right) \\
& \times\left[\hat{b}_{1}^{\dagger}\left(\hat{b}_{2}+\hat{b}_{3}\right)+\hat{b}_{1}\left(\hat{b}_{2}^{\dagger}+\hat{b}_{3}^{\dagger}\right)\right], \\
\hat{I}_{3}= & \hat{b}_{1}^{\dagger 2}\left(\hat{b}_{2}^{2}+\hat{b}_{3}^{2}\right)+\hat{b}_{1}^{2}\left(\hat{b}_{2}^{\dagger 2}+\hat{b}_{3}^{\dagger 2}\right)+\left(\hat{b}_{1}^{\dagger} \hat{b}_{1}+\hat{b}_{1} \hat{b}_{1}^{\dagger}\right) \\
& \times\left[\hat{b}_{2}^{\dagger} \hat{b}_{2}+\hat{b}_{2} \hat{b}_{2}^{\dagger}+\hat{b}_{3}^{\dagger} \hat{b}_{3}+\hat{b}_{3} \hat{b}_{3}^{\dagger}\right], \\
\hat{I}_{4}= & 2\left(\hat{b}_{1}^{\dagger 2} \hat{b}_{2} \hat{b}_{3}+\hat{b}_{1}^{2} \hat{b}_{2}^{\dagger} \hat{b}_{3}^{\dagger}\right)+\left(\hat{b}_{1}^{\dagger} \hat{b}_{1}+\hat{b}_{1} \hat{b}_{1}^{\dagger}\right) \\
& \times\left(\hat{b}_{2}^{\dagger} \hat{b}_{2}+\hat{b}_{2} \hat{b}_{2}^{\dagger}+\hat{b}_{3}^{\dagger} \hat{b}_{3}+\hat{b}_{3} \hat{b}_{3}^{\dagger}\right) \\
& +\hat{b}_{3}^{\dagger} \hat{b}_{1}^{2} \hat{b}_{2}^{\dagger}+\hat{b}_{3} \hat{b}_{1}^{\dagger 2} \hat{b}_{2}+\hat{b}_{3}^{\dagger}\left(\hat{b}_{1}^{\dagger} \hat{b}_{1}+\hat{b}_{1} \hat{b}_{1}^{\dagger}\right) \hat{b}_{2} \\
& +\hat{b}_{3}\left(\hat{b}_{1}^{\dagger} \hat{b}_{1}+\hat{b}_{1} \hat{b}_{1}^{\dagger}\right) \hat{b}_{2}^{\dagger} .
\end{aligned}
$$

The sum of the operators $\hat{\mathcal{O}}_{p}$ involved in the last term of the Hamiltonian (178) runs over the permutations $p=e$, $[120,123]$. Following this approach the spectroscopic parameters are given in terms of the structure and force constants. Note that with this identification the zeroth order Hamiltonian corresponds to a set of noninteracting harmonic oscillators.

The Hamiltonian (178) is diagonalized in a symmetryadapted basis, which is obtained by projecting the functions (159). It has been carried out a fit of the available experimental data $[124,125]$. In Table 6 we present the 21 experimental energies as well as the theoretical energies provided by the fit, where an rms deviation of $1.59 \mathrm{~cm}^{-1}$ was obtained. In the calculation all parameters were freely optimized with equal weights keeping $N=28$ fixed according to dissociation arguments $[47,63]$. The rms provided by the fit in the harmonic limit is $1.62 \mathrm{~cm}^{-1}$, a slightly higher value. From the spectroscopic parameters, the force constants displayed in Table 7 are obtained. In the same table we have included for comparison the parameters obtained by Lukka et al. [122] as well as ab initio results [126]. Although it is possible to obtain a fit of the same quality with less parameters, we have decided to include all the interactions in order to estimate all the available force constants. As noted from Table 7, the results are in good agreement with previous calculations. The discrepancy in the sign of the constant $f_{r r r r}$ is due to the approximation in the potential, where only terms up to quartic order were kept. In our approximation $f_{r r r r}$ must be less than zero in order to reproduce the strong anharmonic behavior in the spectrum, a behavior reproduced naturally when Morse oscillators are considered at zeroth order.

The analysis of transition intensities represents a test for the wave functions provided by any model. In particular in the framework of a local scheme the infrared absorption intensities are calculated with a bond dipole model, where the dipole function is expressed as a sum of bond dipoles in the following form $[11,12]$ :

$$
\vec{\mu}(\mathbf{r})=\sum_{i=1}^{3} \mu_{i}\left(r_{i}\right) \mathbf{e}_{i},
$$

where $r_{i}$ is the instantaneous bond length of the $i$ th bond and $\mathbf{e}_{i}$ is a unit vector along the $i$ th bond. The infrared transition intensities from the initial state $|\nu\rangle$ to the final state $\left|\nu^{\prime}\right\rangle$ within a single electronic state is given by [123]

$$
I_{\nu \rightarrow \nu^{\prime}}=C \Delta E_{\nu v^{\prime}} \sum_{i, j}\left|\mu_{\left(\nu^{\prime}, i\right),(\nu, j)}\right|^{2}
$$

where $C$ is a constant, $\Delta E_{\gamma \gamma^{\prime}}$ is the energy difference between the states, the subindices $\{i, j\}$ account for the degeneracy of the states, while the dipole matrix elements take the form

$$
\begin{aligned}
\left|\mu_{\nu v^{\prime}}\right|^{2} & =\sum_{\zeta=x, y, z}\left|\left\langle v^{\prime}\left|\vec{\mu}(\mathbf{r}) \cdot \mathbf{e}_{\zeta}\right| \nu^{\prime}\right\rangle\right|^{2} \\
& =\sum_{\zeta=x, y, z}\left|\left\langle v^{\prime}\left|\mu_{\zeta}(\mathbf{r})\right| \nu^{\prime}\right\rangle\right|^{2},
\end{aligned}
$$

where the degeneracies have not been considered explicitly. A crucial question that arises is concerned with the form of the bond dipole function. A Taylor series expansion as a function of the bond lengths is a possibility, but a more attractive alternative consists in modeling the local dipole functions with an analytical function, such as the Mecke dipole moment function [127]

$$
\hat{t}_{i}=\mu_{o} q_{i}^{m} e^{-\gamma q_{i}},
$$

where $q_{i}$ is the displacement local coordinate for the $i$ th oscillator, $\gamma$ and $\mu_{o}$ are parameters to be determined, and $m$ is usually taken to be an integer $\geq 1$. Here we will consider $m=1$. The different components of the dipole function is then expanded in terms of the local operators (183).

For pyramidal molecules the dipole operator spans the irreducible representations $A_{1}$ and $E$ in the following form:

$$
\mu_{z}^{A_{1}}, \quad\left(\mu_{x}^{E}, \mu_{y}^{E}\right)
$$

To calculate the intensities, a representation of the dipole operators involved in (181) has to be attained in terms of the local dipole moment functions (183). Projecting according to the chain

$$
\mathcal{C}_{3 v} \supset C_{s}^{a} . \quad \mathcal{C}_{s}^{a}=\left\{E, \sigma_{v}^{a}\right\}
$$


TABLe 6: Energies (in $\mathrm{cm}^{-1}$ ) provided by the fit using the Hamiltonian (178). The rms deviation obtained is $1.59 \mathrm{~cm}^{-1}$. Experimental energies were taken from $[124,125]$.

\begin{tabular}{|c|c|c|c|c|c|c|c|}
\hline Polyad & $\begin{array}{c}\text { State } \\
\text { (normal) }\end{array}$ & $\begin{array}{l}\text { Contribution } \\
\text { (normal) }\end{array}$ & $\begin{array}{l}\text { State } \\
\text { (local) }\end{array}$ & $\begin{array}{c}\text { Contribution } \\
\text { (local) }\end{array}$ & Exp. & $\begin{array}{c}\text { Energies } \\
\text { theor. }\end{array}$ & $\Delta E$ \\
\hline \multicolumn{8}{|c|}{ Symmetry $A_{1}$} \\
\hline 1 & $1_{1}$ & 1.00 & 100 & 1.00 & 2115.16 & 2115.17 & 0.0 \\
\hline 2 & $3_{2}$ & 0.52 & 200 & 0.98 & 4166.77 & 4165.65 & 1.1 \\
\hline 2 & $1_{2}$ & 0.52 & 110 & 0.98 & 4237.7 & 4238.95 & -1.2 \\
\hline 3 & $1_{1} 3_{2}$ & 0.66 & 300 & 0.99 & 6136.34 & 6136.01 & 0.3 \\
\hline 3 & $1_{3}$ & 0.71 & 210 & 0.97 & 6275.83 & 6277.62 & -1.8 \\
\hline 3 & $3_{3}$ & 0.55 & 111 & 0.98 & 6365.95 & 6367.23 & -1.3 \\
\hline 4 & $1_{2} 3_{2}$ & 0.47 & 400 & 0.99 & 8028.98 & 8027.29 & 1.7 \\
\hline 4 & $1_{4}$ & 0.41 & 310 & 0.97 & 8249.51 & 8249.94 & -0.4 \\
\hline 4 & $3_{4}$ & 0.44 & 220 & 0.96 & - & 8332.92 & - \\
\hline 4 & $1_{2} 3_{2}$ & 0.35 & 211 & 0.98 & - & 8397.37 & - \\
\hline 5 & $1_{1} 3_{4}$ & 0.35 & 500 & 0.99 & 9841.4 & 9841.06 & 0.3 \\
\hline 5 & $1_{3} 3_{2}$ & 0.38 & 410 & 0.99 & - & 10143.2 & - \\
\hline 5 & $1_{2} 3_{3}$ & 0.57 & 320 & 0.98 & - & 10289.1 & - \\
\hline 5 & $1_{5}$ & 0.31 & 311 & 0.95 & - & 10370.7 & - \\
\hline 5 & $3_{5}$ & 0.27 & 221 & 0.95 & - & 10443.9 & - \\
\hline 6 & $1_{2} 3_{4}$ & 0.37 & 600 & 0.99 & 11577.5 & 11577.5 & -1.2 \\
\hline 6 & $1_{4} 3_{2}$ & 0.38 & 510 & 0.99 & - & 11957.0 & - \\
\hline 6 & $3_{6}$ & 0.32 & 420 & 0.95 & - & 12183.1 & - \\
\hline 6 & $1_{2} 3_{4}$ & 0.50 & 411 & 0.90 & - & 12265.1 & - \\
\hline 6 & $3_{6}$ & 0.37 & 330 & 0.86 & - & 12270.1 & - \\
\hline 6 & $1_{6}$ & 0.47 & 321 & 0.94 & - & 12401.5 & - \\
\hline 6 & $3_{6}$ & 0.38 & 222 & 0.96 & 一 & 12500.3 & - \\
\hline \multicolumn{8}{|c|}{ Symmetry $A_{2}$} \\
\hline 3 & $3_{3}$ & 1.00 & 210 & 1.00 & - & 6301.12 & - \\
\hline 4 & $1_{1} 3_{3}$ & 1.00 & 310 & 1.00 & - & 8261.71 & - \\
\hline 5 & $1_{2} 3_{3}$ & 0.71 & 410 & 0.99 & - & 10149.9 & - \\
\hline 5 & $3_{5}$ & 0.71 & 320 & 0.99 & - & 10311.7 & - \\
\hline 6 & $1_{1} 3_{5}$ & 0.52 & 510 & 0.99 & - & 11962.9 & - \\
\hline 6 & $1_{3} 3_{3}$ & 0.55 & 420 & 0.99 & - & 12193.7 & - \\
\hline 6 & $3_{6}$ & 0.76 & 321 & 0.99 & 一 & 12439.1 & - \\
\hline \multicolumn{8}{|c|}{ Symmetry $E$} \\
\hline 1 & $3_{1}$ & 1.00 & 100 & 1.00 & 2126.42 & 2126.72 & -0.3 \\
\hline 2 & $1_{1} 3_{1}$ & 0.73 & 200 & 0.99 & 4167.94 & 4168.74 & -0.8 \\
\hline 2 & $3_{2}$ & 0.73 & 011 & 0.99 & 4247.52 & 4248.59 & -1.1 \\
\hline 3 & $1_{2} 3_{1}$ & 0.38 & 300 & 0.99 & 6136.33 & 6136.32 & 0.0 \\
\hline 3 & $1_{2} 3_{1}$ & 0.55 & 012 & 0.99 & 6282.35 & 6283.46 & -1.1 \\
\hline 3 & $3_{3}$ & 0.67 & 120 & 0.99 & 6294.71 & 6295.02 & -0.3 \\
\hline 4 & $1_{1} 3_{3}$ & 0.43 & 400 & 0.99 & 8028.97 & 8027.32 & 1.6 \\
\hline 4 & $1_{3} 3_{1}$ & 0.58 & 310 & 0.98 & 8257.27 & 8253.0 & 4.3 \\
\hline 4 & $1_{2} 3_{2}$ & 0.50 & 130 & 0.98 & 8258.37 & 8258.65 & -0.3 \\
\hline 4 & $3_{4}$ & 0.31 & 022 & 0.97 & - & 8335.51 & - \\
\hline 4 & $3_{4}$ & 0.47 & 211 & 0.99 & - & 8417.15 & - \\
\hline 5 & $1_{2} 3_{3}$ & 0.37 & 500 & 0.99 & 9841.4 & 9841.06 & 0.3 \\
\hline 5 & $1_{4} 3_{1}$ & 0.35 & 410 & 0.99 & - & 10143.7 & - \\
\hline 5 & $1_{1} 3_{4}$ & 0.32 & 140 & 0.99 & - & 10149.8 & - \\
\hline
\end{tabular}


Table 6: Continued.

\begin{tabular}{|c|c|c|c|c|c|c|c|}
\hline Polyad & $\begin{array}{c}\text { State } \\
\text { (normal) }\end{array}$ & $\begin{array}{l}\text { Contribution } \\
\text { (normal) }\end{array}$ & $\begin{array}{l}\text { State } \\
\text { (local) }\end{array}$ & $\begin{array}{l}\text { Contribution } \\
\text { (local) }\end{array}$ & Exp. & $\begin{array}{c}\text { Energies } \\
\text { theor. }\end{array}$ & $\Delta E$ \\
\hline 5 & $1_{3} 3_{2}$ & 0.37 & 023 & 0.98 & - & 10290.4 & - \\
\hline 5 & $3_{5}$ & 0.65 & 230 & 0.99 & - & 10310.4 & - \\
\hline 5 & $1_{2} 3_{3}$ & 0.31 & 311 & 0.98 & - & 10378.1 & - \\
\hline 5 & $3_{5}$ & 0.43 & 122 & 0.98 & - & 10460.9 & - \\
\hline 6 & $1_{3} 3_{3}$ & 0.26 & 600 & 0.99 & 11576.3 & 11577.5 & -1.2 \\
\hline 6 & $1_{5} 3_{1}$ & 0.19 & 510 & 0.99 & - & 11957.1 & - \\
\hline 6 & $1_{2} 3_{4}$ & 0.25 & 150 & 0.99 & - & 11962.8 & - \\
\hline 6 & $1_{5} 3_{1}$ & 0.27 & 024 & 0.95 & - & 12184.1 & - \\
\hline 6 & $1_{1} 3_{5}$ & 0.28 & 240 & 0.99 & - & 12192.7 & - \\
\hline 6 & $1_{1} 3_{5}$ & 0.18 & 411 & 0.94 & - & 12266.3 & - \\
\hline 6 & $3_{6}$ & 0.24 & 033 & 0.91 & - & 12270.1 & - \\
\hline 6 & $1_{2} 3_{4}$ & 0.24 & 123 & 0.99 & - & 12412.6 & - \\
\hline 6 & $1_{1} 3_{5}$ & 0.36 & 231 & 0.99 & - & 12427.1 & - \\
\hline
\end{tabular}

TABle 7: Force constants derived from the spectrocopic parameters given in Table 2. The suface obtained by Lukka was taken from [122], while the ab initio surface from [126].

\begin{tabular}{lccc}
\hline Parameter & This work [65] & Lukka et al. & Ab initio \\
\hline$f_{r r}\left(\mathrm{aJ} \AA^{-2}\right)$ & 2.836 & 2.841 & 2.829 \\
$f_{r r^{\prime}}\left(\mathrm{aJ} \AA^{-2}\right)$ & -0.01040 & -0.00995 & -0.0097 \\
$f_{r r r r}\left(\mathrm{aJ} \AA^{-4}\right)$ & -51.9074 & 46.323 & 54.4 \\
$f_{r r r r^{\prime}}\left(\mathrm{a} J \AA^{-4}\right)$ & 0.2066 & - & - \\
$f_{r r^{\prime} r^{\prime}}\left(\mathrm{a} \AA^{-4}\right)$ & -0.9825 & - & - \\
$f_{r r r^{\prime} r^{\prime \prime}}\left(\mathrm{a} J \AA^{-4}\right)$ & 0.6596 & - & - \\
\hline
\end{tabular}

we obtain the following representation for the dipole components up to first order:

$$
\begin{aligned}
\mu_{x}^{E, A^{\prime}} & =\delta_{1} \frac{1}{\sqrt{6}}\left(2 \hat{t}_{1}-\hat{t}_{2}-\hat{t}_{3}\right), \\
\mu_{y}^{E, A^{\prime \prime}} & =\delta_{1} \frac{1}{\sqrt{2}}\left(\hat{t}_{2}-\hat{t}_{3}\right), \\
\mu_{z}^{A_{1}, A^{\prime}} & =\delta_{2} \frac{1}{\sqrt{3}}\left(\hat{t}_{1}+\hat{t}_{2}+\hat{t}_{3}\right),
\end{aligned}
$$

where $A^{\prime}$ and $A^{\prime \prime}$ label the irreps of the subgroup $\mathcal{C}_{s}^{a}$. We may add contributions of higher order by means of successive couplings. Taking into account this analysis the transition, intensities (181) may be expressed as

$$
\begin{aligned}
I_{v_{i} \rightarrow v_{f}}=\Delta E_{v_{f} v_{i}}\left\{\left|\left\langle v_{f} \Gamma_{f}\left|\mu^{A_{1}}\right| v_{i} \Gamma_{i}\right\rangle\right|^{2}\right. \\
\left.+\left|\left\langle v_{f} \Gamma_{f} A^{\prime}\left|\mu_{x}^{\left(E_{1}, A^{\prime}\right)}\right| v_{i} \Gamma_{i} A^{\prime}\right\rangle\right|^{2}\right\},
\end{aligned}
$$

where we have considered the Wigner-Eckart theorem in order to involve only the first component of the eigenvectors [26].

In order to calculate the matrix elements of the dipole function in the context of the $U(v+1)$ model, it is necessary to obtain an algebraic representation of the operators (183). To achieve this goal we simply substitute the algebraic representation for the coordinates to obtain

$$
\hat{t}_{i}=\mu_{o} \frac{1}{\sqrt{2}}\left(\hat{b}_{i}^{\dagger}+\hat{b}_{i}\right) e^{(-\gamma / \sqrt{2})\left(\hat{b}_{i}^{\dagger}+\hat{b}_{i}\right)},
$$

taking dimensionless units. We thus will be interested in the calculation of the matrix elements of this operators in the local basis (159)

$$
M_{m^{\prime}, m} \equiv\left\langle[N], n^{\prime} ; n_{1}^{\prime}, n_{2}^{\prime}, n_{3}^{\prime}\left|\hat{t}_{i}\right|[N], n ; n_{1}, n_{2}, n_{3}\right\rangle,
$$

where $m$ denotes symbolically the set of local quantum numbers $\left\{N, n, n_{1} n_{2}, n_{3}\right\}$. We can obtain closed analytic results for the matrix elements (189) based on the fact that the operators (165) satisfy the $s u(2)$ commutation relations. Taking advantage of this fact, we can write

$$
e^{(-\gamma / \sqrt{2})\left(\hat{b}_{i}^{\dagger}+\hat{b}_{i}\right)}=e^{-\beta\left(\hat{i}_{i+}+\hat{i}_{i-}\right)}=e^{-\zeta_{1} \hat{i}_{i,+}} e^{-\zeta_{2} \hat{l}_{i-}} e^{-\zeta_{3} \hat{T}_{i, 0}},
$$

where

$$
\begin{aligned}
\beta & =\frac{\gamma}{\sqrt{2 N}}, \\
\zeta_{1} & =\tanh (\beta), \\
\zeta_{2} & =\sinh (\beta) \cosh (\beta), \\
\zeta_{3} & =2 \ln [\cosh (\beta)],
\end{aligned}
$$

which was obtained through a two-dimensional representation of the $s u(2)$ algebra [128]. Hence, the transition operators (188) may be written in the following form:

$$
\hat{t}_{i}=\mu_{0} \frac{1}{\sqrt{2}}\left(\hat{b}_{i}^{\dagger}+\hat{b}_{i}\right) e^{-\left(\zeta_{1} \sqrt{N}\right) \hat{b}_{i}^{\dagger}} e^{-\left(\zeta_{2} \sqrt{N}\right) \hat{b}_{i}} e^{-\left(\zeta_{3} \sqrt{N}\right) \hat{\zeta}_{i, 0}},
$$

where we should take into account that

$$
J_{i, 0}=-\frac{N}{2}\left[1-\frac{1}{N}\left(\hat{n}+\hat{n}_{i}\right)\right]
$$


in accordance with (164). The matrix elements (189) are now able to be calculated in a straightforward way. The result is

$$
\begin{aligned}
M_{m^{\prime}, m}= & \mu_{0} \frac{\left(\Pi_{j \neq i} \delta_{n_{j}^{\prime}, n_{j}}\right)}{\sqrt{2}} e^{\zeta_{3}(N / 2)}\left(-\zeta_{1} \sqrt{N}\right)^{n_{i}^{\prime}-n_{i}-1} \\
& \times \sqrt{n_{i} !\left(n_{i}^{\prime}-1\right) !} \sqrt{n_{i}^{\prime}\left(1-\frac{n^{\prime}-1}{N}\right)} \\
& \times \sum_{q=0}^{n_{i}} \frac{\left(\zeta_{1} \zeta_{2} N\right)^{q}}{q !\left(n_{i}^{\prime}-n_{i}+q-1\right) !\left(n_{i}-q\right) !} \\
& \times f\left(\zeta_{3}, N ; n^{\prime}-1, n ; n_{i}^{\prime}-1, n_{i} ; q\right) \\
& +\mu_{0} \frac{\left(\Pi_{j \neq i} \delta_{n_{j}^{\prime}, n_{j}}\right)}{\sqrt{2}} e^{\zeta_{3} N / 2}\left(-\zeta_{1} \sqrt{N}\right)^{n_{i}^{\prime}-n_{i}+1} \\
& \times \sqrt{n_{i} !\left(n_{i}^{\prime}+1\right) !} \sqrt{\left(n_{i}^{\prime}+1\right)\left(1-\frac{n^{\prime}}{N}\right)} \\
& \times \sum_{q=0}^{n_{i}} \frac{\left(\zeta_{1} \zeta_{2} N\right)^{q}}{q !\left(n_{i}^{\prime}-n_{i}+q+1\right) !\left(n_{i}-q\right) !} \\
& \times f\left(\zeta_{3}, N ; n^{\prime}+1, n ; n_{i}^{\prime}+1, n_{i} ; q\right),
\end{aligned}
$$

where for convenience we have defined

$$
\begin{aligned}
f\left(\zeta_{3}, N ; n^{\prime}, n ; n_{i}^{\prime}, n_{i} ; q\right)= & \frac{e^{\left(\zeta_{3} / 2\right)\left(n+n_{i}\right)}}{N^{q}} \\
& \times \frac{(N-n+q) !}{\sqrt{N^{n_{i}^{\prime}-n_{i}}(N-n) !\left(N-n^{\prime}\right) !}} .
\end{aligned}
$$

This description contrasts with the analysis presented in [47], where the proposed dipole transition operator is based on the general behavior of the dipole transition intensities and has to be defined for each type of local states.

The dipole transition intensities (187) involve three parameters in the linear approximation (186), namely $\left\{\delta_{1}, \delta_{2}, \gamma\right\}$. The parameters $\delta_{1}$ and $\delta_{2}$ fix the scale, while $\gamma$ in (183) determines how the strengths decrease as a function of the number of quanta. Note that since the stretching coordinates are equivalent the operators $\left\{\hat{t}_{i}, i=1,2,3\right\}$ contain the same parameter $\gamma$. These parameters should be determined through a fit to the experiment. The function taken to minimize is

$$
r m s=\left[\frac{\sum_{i=1}^{N_{\exp }}\left[\log \left(I_{\text {exp }}^{i}\right)-\log \left(I_{\text {cal }}^{i}\right)\right]^{2}}{N_{\text {exp }}-N_{\text {par }}}\right]^{1 / 2},
$$

where $N_{\exp }$ and $N_{\text {par }}$ stand for the number of experimental states and the number of parameters used in the fit, respectively. In Table 8 we present the calculated intensities for the experimental data available [124]. The parameters obtained in the fit were

$$
\delta_{1}=0.0173, \quad \delta_{2}=0.0122, \quad \gamma=0.193536 .
$$

The $\delta$ parameters are concerned with the normalization and consequently a small variation does not affect the results considerably. In contrast, $\gamma$ must be specified with high precision. In the same Table 8 , results presented by Halonen et al. [124] and Pluchart et al. [47] are also included. On average our results are similar in accuracy to the ones presented in [124]. This is expected since in essence we are using the same expansion for the dipole operator. However, although at first sight the intensities provided by [47] are better, we should take into account that not all the experimental data were considered and that the number of parameters involved were 4 , one more parameter that is using the linear approximation (186). This treatment to describe dipole transition intensities is general and is readily extended to include higher-order expansions in the dipole operators, which in the harmonic limit reduce to the usual expansions considered in local mode models [11].

\section{Algebraic Approach to Describe Linear Triatomic Molecules}

The unitary model $U(3)$ deserves special attention because it is suitable to be applied to the description of the degenerate bending degrees of freedom of linear molecules. In fact, the use of the $s u(3)$ algebra for the bends and the $s u(2)$ algebras for the stretches turns out to be a successful scheme to describe the vibrational excitations of linear molecules.

In this section we present the procedure to estimate the PES when the $s u(2) \times s u(3) \times s u(2)$ Lie algebra is used as the dynamical group of linear triatomic molecules. In this scheme, each stretching degree of freedom is described through an $s u(2)$ dynamical algebra, while the doubly degenerate bending degree of freedom is modeled with an $s u(3)$ dynamical algebra. Again to achieve this goal we establish the connection between the traditional approach and the algebraic method. As an application, the analysis of the vibrational excitations of $\mathrm{CO}_{2}$ in its ground electronic state is considered.

As we know in terms of internal displacement coordinates, $q_{k}$, the quantum mechanical Hamiltonian that describes the vibrational excitations of a molecule takes the form

$$
H=\frac{1}{2} \widetilde{\mathbf{p}} \mathbf{G}(\mathbf{q}) \mathbf{p}+V(\mathbf{q})
$$

The explicit form of the Hamiltonian strongly depends on the molecular system. In the case of linear triatomic molecules, two sets of internal coordinates are needed. The first set, $\left(q_{1}, q_{2}\right)$, spans the subspace of stretching vibrations with

$$
q_{i}=\Delta r_{i}=r_{i}-r_{e}, \quad i=1,2,
$$

where $r_{i}$ corresponds to the $i$ th bond length and $r_{e}$ to the $i$ th bond equilibrium length. The second set, $\left(q_{a}, q_{b}\right)$, spans the subspace of bending oscillators with the following definition [129]:

$$
q_{a}=r_{e} \mathbf{e}_{Y} \cdot \frac{\mathbf{r}_{1} \times \mathbf{r}_{2}}{r_{1} r_{2}}, \quad q_{b}=-r_{e} \mathbf{e}_{X} \cdot \frac{\mathbf{r}_{1} \times \mathbf{r}_{2}}{r_{1} r_{2}},
$$


TABLE 8: Observed and calculated relative intensities of the stretching vibrational bands in arsine from [65].

\begin{tabular}{|c|c|c|c|c|c|}
\hline Local state & $v_{\text {calc }} / \mathrm{cm}^{-1}$ & $I_{\mathrm{obs}}$ & $I_{\text {calc }}$ & Halonen et al. [124] & Pluchart et al. [47] \\
\hline \multicolumn{6}{|c|}{ Symmetry $A_{1}$} \\
\hline$(100)$ & 2115.13 & $1.0^{\mathrm{a}}$ & 1.0 & 1 & 1.0 \\
\hline$(200)$ & 4165.79 & 0.021 & 0.068 & 0.022 & 0.0209 \\
\hline (110) & 4238.94 & - & $1.14 \times 10^{-3}$ & $0.13 \times 10^{-3}$ & - \\
\hline$(300)$ & 6136.07 & $0.32 \times 10^{-3}$ & $1.32 \times 10^{-3}$ & $0.49 \times 10^{-3}$ & $0.47 \times 10^{-3}$ \\
\hline$(210)$ & 6277.63 & $0.28 \times 10^{-4}$ & $0.07 \times 10^{-4}$ & $0.0055 \times 10^{-4}$ & - \\
\hline$(400)$ & 8027.32 & $0.15 \times 10^{-4}$ & $0.129 \times 10^{-4}$ & $0.071 \times 10^{-4}$ & $0.12 \times 10^{-4}$ \\
\hline \multicolumn{6}{|c|}{ Symmetry $E$} \\
\hline$(100)$ & 2126.81 & $1.0^{\mathrm{a}}$ & 1.0 & 1.0 & 1.0 \\
\hline$(200)$ & 4168.75 & 0.021 & 0.069 & 0.022 & 0.0209 \\
\hline$(011)$ & 4248.66 & - & $2.64 \times 10^{-4}$ & $0.58 \times 10^{-4}$ & - \\
\hline$(300)$ & 6136.36 & $0.32 \times 10^{-3}$ & $1.32 \times 10^{-3}$ & $0.49 \times 10^{-3}$ & $0.47 \times 10^{-3}$ \\
\hline$(012)$ & 6283.43 & - & $1.51 \times 10^{-6}$ & $0.51 \times 0^{-6}$ & - \\
\hline (120) & 6295.01 & $0.14 \times 10^{-4}$ & $0.015 \times 10^{-4}$ & $0.0032 \times 10^{-4}$ & - \\
\hline$(400)$ & 8027.35 & $0.15 \times 10^{-4}$ & $0.128 \times 10^{-4}$ & $0.071 \times 10^{-4}$ & $0.12 \times 10^{-4}$ \\
\hline
\end{tabular}

${ }^{\mathrm{a}}$ Scaled value.

where $\mathbf{r}_{1}$ and $\mathbf{r}_{2}$ are vectors from the C-atom to each one of the $\mathrm{O}$-atoms. The unit vectors $\mathbf{e}_{X}$ and $\mathbf{e}_{Y}$ lie on the direction of the $x$ and $y$-axes of the laboratory axis system, with its origin in the molecule's center of mass.

With this choice of coordinates, the $\mathbf{q}$ and $\mathbf{p}$ vectors in (198) are given by

$$
\widetilde{\mathbf{q}}=\left(q_{1}, q_{2}, q_{a}, q_{b}\right), \quad \widetilde{\mathbf{p}}=\left(p_{1}, p_{2}, p_{a}, p_{b}\right) .
$$

In general for linear molecules the matrix $\mathbf{G}(\mathbf{q})$ cannot be obtained in closed form. However, it is possible to evaluate the G-matrix as well as its derivatives at equilibrium using its explicit form in terms of the Cartesian coordinates. This procedure is presented in [69].

We could expand the Wilson matrix as well as the potential energy in terms of the local coordinates. This expansion permits the identification of a set of local interacting oscillators in (198). This approach, however, is largely improved when symmetry-adapted coordinates are first introduced.

Hence, we start introducing the symmetry coordinates suitable for homonuclear molecules

$$
\begin{aligned}
\mathcal{Q}_{\Sigma_{g}}=\frac{1}{\sqrt{2}}\left(q_{1}+q_{2}\right), & \mathcal{Q}_{\Sigma_{u}}=\frac{1}{\sqrt{2}}\left(q_{1}-q_{2}\right), \\
\mathcal{Q}_{+}=-\frac{1}{\sqrt{2}}\left(q_{a}+i q_{b}\right), & \mathcal{Q}_{-}=\frac{1}{\sqrt{2}}\left(q_{a}-i q_{b}\right),
\end{aligned}
$$

where the coordinates associated with the stretches are labeled with the subscripts $\Sigma_{g / u}$, and the bending degrees of freedom with $+/-$. The first case is a simplification of $\Sigma_{g / u}^{+}$, while the bending labels are a shorthand notation for $\Pi^{ \pm}$ $[8,130]$. Both labels refer to the irreducible representations of the point symmetry group $\mathscr{D}_{\infty h}$. In matrix form

$$
\widehat{\mathcal{Q}}=\tilde{\mathbf{q}} S,
$$

where

$$
\begin{aligned}
& \hat{\mathcal{Q}}=\left(\mathcal{Q}_{\Sigma_{u}}, \mathcal{Q}_{\Sigma_{g}}, \mathcal{Q}_{-}, \mathcal{Q}_{+}\right), \\
& S=\frac{1}{\sqrt{2}}\left(\begin{array}{cccc}
1 & 1 & 0 & 0 \\
-1 & 1 & 0 & 0 \\
0 & 0 & 1 & -1 \\
0 & 0 & -i & -i
\end{array}\right) .
\end{aligned}
$$

The change to symmetry coordinates transforms the Hamiltonian (198) into

$$
\hat{H}=\frac{1}{2} \widehat{\mathcal{P}} g \mathcal{P}+V(\mathcal{Q})
$$

where

$$
\begin{gathered}
\widehat{\mathcal{P}}=\left(\mathcal{P}_{\Sigma_{u}}, \mathcal{P}_{\Sigma_{g}}, \mathcal{P}_{-}, \mathcal{P}_{+}\right), \\
g=\widetilde{\mathbf{S} G S} .
\end{gathered}
$$

The transformed Wilson matrix $g$ is block diagonal, as expected.

We may now proceed to expand the Wilson Matrix as well as the potential in terms of the symmetry-adapted coordinates, keeping the relevant terms associated with the resonances. It is thus necessary to establish the polyad corresponding to the system of interest, in this case for the $\mathrm{CO}_{2}$ molecule. The fundamentals of $\mathrm{CO}_{2}$ are $\left(\mathrm{in} \mathrm{cm}^{-1}\right)$ [8]

$$
\nu_{1}=1285.41, \quad v_{2}=667.38, \quad \nu_{3}=2349.14,
$$

from which we set up the following allowed symmetry resonances:

$$
v_{1} \approx 2 v_{2} .
$$


The identification of these resonances permits to establish the polyad $P$, which in terms of integer numbers may be defined as

$$
P=2\left(\nu_{1}+v_{3}\right)+v_{2}
$$

The expansion of the $g$ matrix and the potential in terms of the symmetry coordinates leads to a Hamiltonian of the form [68]

$$
\hat{H}=\hat{H}_{s}+\hat{H}_{b}+\hat{H}_{s b},
$$

where the first term, $\hat{H}_{s}$, is the pure stretching contribution

$$
\begin{aligned}
\hat{H}_{s}= & \frac{1}{2} g_{\Sigma_{g} \Sigma_{g}}^{0} \widehat{\mathcal{P}}_{\Sigma_{g}}^{2}+\frac{1}{2} g_{\Sigma_{u} \Sigma_{u}}^{0} \widehat{\mathcal{P}}_{\Sigma_{u}}^{2}+\frac{1}{2} f_{\Sigma_{g} \Sigma_{g}} \widehat{\mathcal{Q}}_{\Sigma_{g}}^{2}+\frac{1}{2} f_{\Sigma_{u} \Sigma_{u}} \widehat{\mathcal{Q}}_{\Sigma_{u}}^{2} \\
& +\frac{1}{4 !} f_{\Sigma_{g} \Sigma_{g} \Sigma_{g} \Sigma_{g}} \widehat{\mathcal{Q}}_{\Sigma_{g}}^{4}+\frac{1}{4 !} f_{\Sigma_{u} \Sigma_{u} \Sigma_{u} \Sigma_{u}} \widehat{\mathcal{Q}}_{\Sigma_{u}}^{4}+\frac{6}{4 !} f_{\Sigma_{g} \Sigma_{g} \Sigma_{u} \Sigma_{u}} \widehat{\mathcal{Q}}_{\Sigma_{g}}^{2} \widehat{\mathcal{Q}}_{\Sigma_{u}}^{2} .
\end{aligned}
$$

The second term, $\hat{H}_{b}$, depends solely on bending coordinates and momenta

$$
\begin{aligned}
\hat{H}_{b}= & g_{+-}^{0} \widehat{\mathcal{P}}_{+} \widehat{\mathcal{P}}_{-}+f_{+-} \hat{\mathcal{Q}}_{+} \hat{\mathcal{Q}}_{-}+\left(\frac{\partial^{2} g_{+-}}{\partial \mathcal{Q}_{+} \partial \mathcal{Q}_{-}}\right)_{0} \widehat{\mathcal{P}}_{+} \widehat{\mathcal{Q}}_{+} \widehat{\mathcal{Q}}_{-} \widehat{\mathcal{P}}_{-} \\
& +\frac{6}{4 !} f_{++--} \hat{\mathcal{Q}}_{+}^{2} \widehat{\mathcal{Q}}_{-}^{2},
\end{aligned}
$$

while, the third term, $\hat{H}_{s b}$, embodies the stretch-bend interaction terms

$$
\begin{aligned}
& \hat{H}_{s b}=\left(\frac{\partial g_{+-}}{\partial \mathcal{Q}_{\Sigma_{g}}}\right)_{0} \mathcal{Q}_{\Sigma_{g}} \widehat{\mathcal{P}}_{+} \widehat{\mathcal{P}}_{-}+\left(\frac{\partial g_{\Sigma_{g}+}}{\partial \mathcal{Q}_{+}}\right) \widehat{Q}_{0}\left(\widehat{Q}_{\Sigma_{g}} \widehat{\mathcal{P}}_{+}+\widehat{\mathcal{P}}_{-} \widehat{Q}_{-}\right) \\
& +\frac{6}{3 !} f_{\Sigma_{g}+-} \hat{Q}_{\Sigma_{g}} \widehat{Q}_{+} \hat{Q}_{-}+\frac{1}{2}\left(\frac{\partial^{2} g_{+-}}{\partial \mathcal{Q}_{\Sigma_{g}}^{2}}\right)_{0} \hat{Q}_{\Sigma_{g}}^{2} \widehat{\mathcal{P}}_{+} \widehat{\mathcal{P}}_{-} \\
& +\frac{1}{2}\left(\frac{\partial^{2} g_{+-}}{\partial Q_{\Sigma_{u}}^{2}}\right)_{0} \widehat{Q}_{\Sigma_{u}}^{2} \widehat{\mathcal{P}}_{+} \widehat{\mathcal{P}}_{-} \\
& +\frac{1}{2}\left(\frac{\partial^{2} g_{\Sigma_{g} \Sigma_{g}}}{\partial \mathcal{Q}_{+} \partial Q_{-}}\right) \widehat{\mathcal{P}}_{\Sigma_{g}}^{2} \widehat{Q}_{+} \widehat{Q}_{-}+\frac{1}{2}\left(\frac{\partial^{2} g_{\Sigma_{u} \Sigma_{u}}}{\partial \mathcal{Q}_{+} \partial \mathcal{Q}_{-}}\right) \widehat{\mathcal{P}}_{\Sigma_{u}}^{2} \widehat{Q}_{+} \widehat{Q}_{-} \\
& +\frac{1}{2}\left(\frac{\partial^{2} g_{\Sigma_{g}+}}{\partial \mathcal{Q}_{\Sigma_{g}} \partial \mathcal{Q}_{+}}\right)_{0} \widehat{\mathcal{P}}_{\Sigma_{g}} \widehat{Q}_{\Sigma_{g}}\left(\widehat{Q}_{+} \widehat{\mathcal{P}}_{+}+\widehat{Q}_{-} \widehat{\mathcal{P}}_{-}\right) \\
& +\frac{1}{2}\left(\frac{\partial^{2} g_{\Sigma_{u}+}}{\partial \mathcal{Q}_{\Sigma_{u}} \partial \mathcal{Q}_{+}}\right) \widehat{\mathcal{P}}_{\Sigma_{u}} \widehat{Q}_{\Sigma_{u}}\left(\widehat{Q}_{+} \widehat{\mathcal{P}}_{+}+\widehat{Q}_{-} \widehat{\mathcal{P}}_{-}\right) \\
& +\frac{12}{4 !} f_{\Sigma_{g} \Sigma_{g}+-} \hat{\mathcal{O}}_{\Sigma_{g}}^{2} \hat{Q}_{+} \hat{\mathcal{Q}}_{-} \\
& +\frac{12}{4 !} f_{\Sigma_{u} \Sigma_{u}+-} \hat{\mathcal{Q}}_{\Sigma_{u}}^{2} \hat{\mathcal{Q}}_{+} \hat{\mathcal{Q}}_{-} .
\end{aligned}
$$

The Hamiltonian (210) can be translated into an algebraic representation through the introduction of bosonic creation and annihilation operators. For the stretching degrees of freedom, these operators are defined as

$$
a_{\Gamma}^{\dagger}=\alpha^{\Gamma} \widehat{\mathcal{Q}}_{\Gamma}-\frac{i}{2 \hbar \alpha^{\Gamma}} \widehat{\mathcal{P}}_{\Gamma}, \quad a_{\Gamma}=\alpha^{\Gamma} \widehat{\mathcal{Q}}_{\Gamma}+\frac{i}{2 \hbar \alpha^{\Gamma}} \widehat{\mathcal{P}}_{\Gamma},
$$

where $\Gamma=\Sigma_{g}, \Sigma_{u}$ and the definitions

$$
\begin{aligned}
& \left(\alpha^{\Sigma_{g}}\right)^{2}=\frac{1}{2 \hbar} \sqrt{\frac{f_{\Sigma_{g} \Sigma_{g}}}{g_{\Sigma_{g} \Sigma_{g}}^{0}}}=\frac{\mu_{\Sigma_{g}}}{2 \hbar} \sqrt{f_{\Sigma_{g} \Sigma_{g}} g_{\Sigma_{g} \Sigma_{g}}^{0}}=\frac{\omega_{\Sigma_{g}} \mu_{\Sigma_{g}}}{2 \hbar}, \\
& \left(\alpha^{\Sigma_{u}}\right)^{2}=\frac{1}{2 \hbar} \sqrt{\frac{f_{\Sigma_{u} \Sigma_{u}}}{g_{\Sigma_{u} \Sigma_{u}}^{0}}}=\frac{\mu \Sigma_{\Sigma_{u}}}{2 \hbar} \sqrt{f_{\Sigma_{u} \Sigma_{u} g_{\Sigma_{u} \Sigma_{u}}^{0}}^{0}}=\frac{\omega_{\Sigma_{u}} \mu_{\Sigma_{u}}}{2 \hbar},
\end{aligned}
$$

where $\mu_{\Gamma}=1 / g_{\Gamma \Gamma}^{0}$. In the case of the bending mode, the following relations apply:

$$
a_{ \pm}^{\dagger}=\alpha^{ \pm} \widehat{Q}_{ \pm}+\frac{i}{2 \hbar \alpha^{ \pm}} \widehat{\mathcal{P}}_{\mp}, \quad a_{ \pm}=-\alpha^{ \pm} \mathcal{Q}_{\mp}+\frac{i}{2 \hbar \alpha^{ \pm}} \widehat{\mathcal{P}}_{ \pm},
$$

with

$$
\left(\alpha^{+}\right)^{2}=\left(\alpha^{-}\right)^{2}=(\alpha)^{2}=\frac{1}{2 \hbar} \sqrt{\frac{f_{+-}}{g_{+-}^{0}}}=\frac{1}{2 \hbar} \sqrt{\frac{f_{q_{a} q_{a}}}{g_{q_{a} q_{a}}^{0}}} .
$$

We now carry out the additional canonical transformation between symmetrized and local stretching bosonic operators (67)

$$
a_{\Sigma_{g}}^{\dagger}=\frac{1}{\sqrt{2}}\left(c_{1}^{\dagger}+c_{2}^{\dagger}\right), \quad a_{\Sigma_{u}}^{\dagger}=\frac{1}{\sqrt{2}}\left(c_{1}^{\dagger}-c_{2}^{\dagger}\right) .
$$

The operators $\left\{c_{i}^{\dagger}\right\}$, with $i=1,2$, will be identified with local operators in accordance with (70). If we restrict the interactions to preserve the polyad number

$$
P=2\left(n_{\Sigma_{g}}+n_{\Sigma_{u}}\right)+\left(n_{+}+n_{-}\right),
$$

where $n_{\Sigma_{g} / u}$ stands for the number of quanta in the $\Sigma_{g / u}$ vibrational mode and $n_{+}+n_{-}=n$ is the number of quanta in the degenerate bending mode, the Hamiltonian (210) in the local harmonic representation is

$$
\begin{aligned}
\hat{H}= & \widetilde{\omega}_{s} \sum_{i=1}^{2}\left(a_{i}^{\dagger} a_{i}+a_{i} a_{i}^{\dagger}\right)+\lambda_{s} \sum_{i \neq j=1}^{2} a_{i}^{\dagger} a_{j}+\alpha_{1}^{s}\left(\hat{n}_{1}^{2}+\hat{n}_{2}^{2}\right) \\
& +\alpha_{2}^{s}\left(a_{1}^{\dagger 2} a_{2}^{2}+a_{2}^{\dagger 2} a_{1}^{2}+4 \hat{n}_{1} \hat{n}_{2}\right) \\
& +\alpha_{3}^{s}\left(\hat{n}_{1} a_{2}^{\dagger} a_{1}+\hat{n}_{2} a_{1}^{\dagger} a_{2}+\text { H.c. }\right) \\
& +\widetilde{\omega}_{b} \hat{n}+\alpha_{1}^{b} \hat{n}^{2}+\alpha_{2}^{b} \hat{\ell}^{2}+\alpha_{1}^{s b}\left\{\left(a_{1}^{\dagger}+a_{2}^{\dagger}\right) a_{+} a_{-}+\text {H.c. }\right\} \\
& +\alpha_{2}^{s b}\left(\hat{n}_{1}+\hat{n}_{2}\right) \hat{n}+\alpha_{3}^{s b}\left(a_{1}^{\dagger} a_{2}+a_{2}^{\dagger} a_{1}\right) \hat{n},
\end{aligned}
$$


with the definitions

$$
\begin{gathered}
\hat{n}_{i}=a_{i}^{\dagger} a_{i}, \quad i=1,2, \\
\hat{\ell}=a_{+}^{\dagger} a_{+}-a_{-}^{\dagger} a_{-}=\hat{n}_{+}-\hat{n}_{-}, \\
\hat{n}=a_{+}^{\dagger} a_{+}+a_{-}^{\dagger} a_{-}=\hat{n}_{+}+\hat{n}_{-},
\end{gathered}
$$

where $\hat{n}_{i}$ is the number of quanta for the $i$-th stretching oscillator, $\hat{n}$ is the total number of bending quanta, and $\hat{\ell}$ is the vibrational angular momentum. The expressions for the spectroscopic parameters in terms of the structure and force constants are detailed in Appendix C of [68]. An appropriate basis to diagonalize the Hamiltonian (220) is constructed by means of the symmetry projection of the direct product of functions

$$
\left|n_{1} n_{2}, n^{\ell}\right\rangle=\mathcal{N}\left(a_{1}^{\dagger}\right)^{n_{1}}\left(a_{2}^{\dagger}\right)^{n_{2}}\left(a_{+}^{\dagger}\right)^{(n+\ell) / 2}\left(a_{-}^{\dagger}\right)^{(n-\ell) / 2}|0\rangle,
$$

with normalization constant

$$
\mathcal{N}=\frac{1}{\sqrt{n_{1} ! n_{2} !((n+\ell) / 2) !((n-\ell) / 2) !}} .
$$

The present treatment is based on local harmonic oscillator. The next step consists in introducing the anharmonization as in the previous models.

Let us first proceed to introduce the algebraic approach based on unitary groups. An algebraic description of a system consists in identifying an appropriate dynamical algebra. A fundamental feature of this approach is that the system's Hilbert space carries a specific irreducible representation of the dynamical algebra. In $\mathrm{CO}_{2}$ the stretching vibrational modes will be associated with $s u(2)$ algebras, while an $s u(3)$ dynamical algebra is proposed to describe the vibrational bending modes. The result is a simple model able to cope with the rigidly linear and rigidly bent limits as well as the situations in between these two limits [34-38]. The dynamical group for the complete triatomic molecule is then given by $S U_{1}(2) \times S U(3) \times S U_{2}(2)[131,132]$.

The bosonic $s u(3)$ Lie algebra is not related to a known potential. It can be constructed with two Cartesian boson creation and annihilation operators $\left\{\tau_{a}^{\dagger}, \tau_{b}^{\dagger}, \tau_{a}, \tau_{b}\right\}$, together with a scalar boson $\left\{\sigma^{\dagger}, \sigma\right\}[23,24]$. To be consistent with (200), we label the Cartesian operators as $a$ and $b$ instead of $x$ and $y$. From the physical point of view it is convenient to introduce spherical (circular) bosons

$$
\tau_{ \pm}^{\dagger}=\mp \frac{\tau_{a}^{\dagger} \pm i \tau_{b}^{\dagger}}{\sqrt{2}}, \quad \tau_{ \pm}=\mp \frac{\tau_{a} \mp i \tau_{b}}{\sqrt{2}},
$$

since they carry irreducible representations of the symmetry group $D_{\infty h}$. Following $[23,24]$, the nine generators of $U(3)$ may be written as combinations of the bilinear products of a creation and annihilation operator:

$$
\begin{gathered}
\hat{n}=\tau_{+}^{\dagger} \tau_{+}+\tau_{-}^{\dagger} \tau_{-}, \quad \hat{n}_{\sigma}=\sigma^{\dagger} \sigma, \\
\hat{l}=\tau_{+}^{\dagger} \tau_{+}-\tau_{-}^{\dagger} \tau_{-}, \\
\hat{D}_{+}=\sqrt{2}\left(\tau_{+}^{\dagger} \sigma-\sigma^{\dagger} \tau_{-}\right), \quad \hat{D}_{-}=\sqrt{2}\left(-\tau_{-}^{\dagger} \sigma+\sigma^{\dagger} \tau_{+}\right), \\
\hat{Q}_{+}=\sqrt{2} \tau_{+}^{\dagger} \tau_{-}, \quad \hat{Q}_{-}=\sqrt{2} \tau_{-}^{\dagger} \tau_{+}, \\
\hat{R}_{+}=\sqrt{2}\left(\tau_{+}^{\dagger} \sigma+\sigma^{\dagger} \tau_{-}\right), \quad \hat{R}_{-}=\sqrt{2}\left(\tau_{-}^{\dagger} \sigma+\sigma^{\dagger} \tau_{+}\right) .
\end{gathered}
$$

The operator $\hat{l}$ is identified as the $2 \mathrm{D}$ angular momentum in (221). There are two possible chains starting from $S U(3)$ and ending in $S O(2)$ (i.e., that conserve $2 \mathrm{D}$ angular momentum):

$$
\begin{gathered}
U(3) \supset U(2) \supset S O(2) \longrightarrow \text { Chain }(\mathrm{Ib}), \\
U(3) \supset S O(3) \supset S O(2) \longrightarrow \text { Chain }(\mathrm{IIb}),
\end{gathered}
$$

where the $b$ label stands for bending. The corresponding subalgebras are composed by the following elements:

$$
\begin{gathered}
U(2)\left\{\hat{n}, \hat{l}, \hat{Q}_{+}, \hat{Q}_{-}\right\}, \\
S O(3)\left\{\hat{l}, \hat{D}_{+}, \hat{D}_{-}\right\}, \\
S O(2)\{\hat{l}\} .
\end{gathered}
$$

Following the algebraic methodology, the Hamiltonian is expanded in terms of powers and products of the Casimir operators. However, in molecular systems, if the Hamiltonian goes beyond one- and two-body operators, the number of interactions is greater than the number of invariant operators [133]. Consequently it is convenient to use another prescription to construct the Hamiltonian. One possibility consists in expanding the Hamiltonian in terms of the dynamical algebra $[131,132]$, but another alternative consists in establishing the connection between the coordinates and momenta and the generators of the dynamical algebra in such a way that all the algebraic interactions are extracted from the Hamiltonian in configuration space. In any case a suitable bending basis to diagonalize the Hamiltonian is given by

$$
\left|[N] ; n_{\sigma}, n^{l}\right\rangle=\mathcal{N}_{b}\left(\sigma^{\dagger}\right)^{n_{\sigma}}\left(\tau_{+}^{\dagger}\right)^{(n+l) / 2}\left(\tau_{-}^{\dagger}\right)^{(n-l) / 2}|0\rangle,
$$

with normalization constant

$$
\mathcal{N}_{b}=\frac{1}{\sqrt{n_{\sigma} !((n+l) / 2) !((n-l) / 2) !}} .
$$

Note that the kets (228) may be denoted as

$$
\left|[N] ; n_{\sigma}, n^{l}\right\rangle=\left|[N] ; n^{l}\right\rangle,
$$

because of the constraint $\hat{N}=\hat{n}+\hat{n}_{\sigma}$. 
Now we follow an approach starting from the Hamiltonian (210). Following the approach given in $[63,64]$, from the generators (225) of the unitary group $U(3)$ we identify two $s u_{k}(2)$ subalgebras with generators

$$
\begin{gathered}
\hat{J}_{+, k}=\sigma \tau_{k}^{\dagger}, \\
\hat{J}_{-, k}=\sigma^{\dagger} \tau_{k}, \\
\hat{J}_{0, k}=-\frac{1}{2}\left(\sigma^{\dagger} \sigma-\hat{n}_{k}\right), \quad k=a, b,
\end{gathered}
$$

where $\hat{n}_{k}=\tau_{k}^{\dagger} \tau_{k}$. The operators $\left\{\hat{J}_{ \pm, k}, \hat{J}_{0, k}\right\}$ have the usual angular momentum commutation relations [73]. We now introduce the normalized operators

$$
b_{k}^{\dagger} \equiv \frac{\hat{J}_{+, k}}{\sqrt{N}}, \quad b_{k} \equiv \frac{\hat{J}_{-, k}}{\sqrt{N}}, \quad k=a, b,
$$

and the circular operators

$$
b_{ \pm}^{\dagger}=\mp \frac{b_{a}^{\dagger} \pm i b_{b}^{\dagger}}{\sqrt{2}}, \quad b_{ \pm}=\mp \frac{b_{a} \mp i b_{b}}{\sqrt{2}} .
$$

The action of these operators over the kets (230) is the following

$$
\begin{aligned}
& b_{ \pm}^{\dagger}\left|[N] ; n^{l}\right\rangle=\sqrt{\left(\frac{n \pm l}{2}+1\right)\left(1-\frac{n}{N}\right)}\left|[N] ;(n+1)^{l \pm 1}\right\rangle, \\
& b_{ \pm}\left|[N] ; n^{l}\right\rangle=\sqrt{\left(\frac{n \pm l}{2}\right)\left(1-\frac{n-1}{N}\right)}\left|[N] ;(n-1)^{l \mp 1}\right\rangle .
\end{aligned}
$$

The harmonic limit is obtained taking the $N$ large limit [68]

$$
\lim _{N \rightarrow \infty} b_{ \pm}^{\dagger}=\tau_{ \pm}^{\dagger}, \quad \lim _{N \rightarrow \infty} b_{ \pm}=\tau_{ \pm} .
$$

Keeping in mind the estimation of the PES, it is crucial to establish the connection between the generators of the dynamical algebra and the local coordinates and momenta, at least in an approximate form. We propose the following approximation for the local coordinates, $\widehat{\mathcal{Q}}_{ \pm}$, and momenta, $\widehat{\mathcal{P}}_{ \pm}$, in the framework of the $U(3)$ model [68]

$\widehat{Q}_{ \pm} \simeq \sqrt{\frac{\hbar}{2 \omega_{b} \mu_{b}}}\left(d_{ \pm}^{\dagger}-d_{\mp}\right), \quad \widehat{\mathcal{P}}_{ \pm} \simeq-\frac{i}{2} \sqrt{2 \hbar \omega_{b} \mu_{b}}\left(d_{\mp}^{\dagger}+d_{ \pm}\right)$,

where $d_{ \pm}^{\dagger}\left(d_{ \pm}\right)$may be identified either with $b_{ \pm}^{\dagger}\left(b_{ \pm}\right)$or $a_{ \pm}^{\dagger}\left(a_{ \pm}\right)$, following the prescription discussed in [63-66]. Here the parameters $\omega_{b}$ and $\mu_{b}$ are $\omega_{b}=\sqrt{f_{+-} g_{+-}^{0}}=$ $\sqrt{f_{q_{a} q_{a} g_{q_{a} q_{a}}^{0}}}$ and $\mu_{b}=1 / g_{+-}^{0}=1 / g_{q_{a} q_{a}}^{0}$.

The proposed relation (236) represents just an approximation, not only because we are truncating to a linear expansion in terms of the operators (233), but also because the commutation relations,

$$
\left[\widehat{Q}_{j}, \widehat{\mathcal{P}}_{k}\right]=i \hbar \delta_{j k}
$$

have a correction of the order $1 / N$ when the operators $d_{ \pm}^{\dagger}\left(d_{ \pm}\right)$ are substituted by $b_{ \pm}^{\dagger}\left(b_{ \pm}\right)$[68]. We now suggest to use the relations (236) instead of (216), with the substitution of the operators $d_{ \pm}^{\dagger}\left(d_{ \pm}\right)$by $a_{ \pm}^{\dagger}\left(a_{ \pm}\right)$when number and angular momentum operators are involved. Otherwise, we use the substitution rule $d_{ \pm}^{\dagger}\left(d_{ \pm}\right) \rightarrow b_{ \pm}^{\dagger}\left(b_{ \pm}\right)$, as it is the case for Fermi interactions [63-66]. With regard to the stretching degrees of freedom, we may consider the anharmonization procedure

$$
a_{i}^{\dagger} \longrightarrow b_{i}^{\dagger}, \quad a_{i} \longrightarrow b_{i}, \quad i=1,2,
$$

as discussed in Section 6.

Following this approach, the following Hamiltonian is obtained:

$$
\begin{aligned}
\hat{H}= & \widetilde{\omega}_{s} \sum_{i=1}^{2}\left(b_{i}^{\dagger} b_{i}+b_{i} b_{i}^{\dagger}\right)+\lambda_{s} \sum_{i \neq j=1}^{2} b_{i}^{\dagger} b_{j}+\alpha_{1}^{s}\left(\hat{n}_{s, 1}^{2}+\hat{n}_{s, 2}^{2}\right) \\
& +\alpha_{2}^{s}\left(b_{1}^{\dagger 2} b_{2}^{2}+b_{2}^{\dagger 2} b_{1}^{2}+4 \hat{n}_{s, 1} \hat{n}_{s, 2}\right) \\
& +\alpha_{3}^{s}\left(\hat{n}_{s, 1} b_{2}^{\dagger} b_{1}+\hat{n}_{s, 2} b_{1}^{\dagger} b_{2}+H . c .\right) \\
& +\widetilde{\omega}_{b} \hat{n}+\alpha_{1}^{b} \hat{n}^{2}+\alpha_{2}^{b} \hat{\ell}^{2}+\alpha_{1}^{s b}\left\{\left(b_{1}^{\dagger}+b_{2}^{\dagger}\right) b_{+} b_{-}+H . c .\right\} \\
& +\alpha_{2}^{s b}\left(\hat{n}_{s, 1}+\hat{n}_{s, 2}\right) \hat{n}+\alpha_{3}^{s b}\left(b_{1}^{\dagger} b_{2}+b_{2}^{\dagger} b_{1}\right) \hat{n},
\end{aligned}
$$

with the definition

$$
\hat{n}_{s, i}=b_{i}^{\dagger} b_{i}, \quad i=1,2 .
$$

The equations that relate the spectroscopic parameters and force constants are given in Appendix C of [68]. In order to carry out the necessary calculations, we select the basis

$$
\left|\left[N_{s}\right],[N] ; v_{1} v_{2} ; n^{\ell}\right\rangle=\left|\left[N_{s}\right] ; v_{1} v_{2}\right\rangle \otimes\left|[N] ; n^{\ell}\right\rangle,
$$

where $\left|[N] ; n^{\ell}\right\rangle$ is given by (228) and for the stretches

$$
\left|\left[N_{s}\right] ; v_{1} v_{2}\right\rangle=\left|\left[N_{s}\right] ; v_{1}\right\rangle \otimes\left|\left[N_{s}\right] ; v_{2}\right\rangle,
$$

with

$$
\left|\left[N_{s}\right] ; v_{i}\right\rangle=\sqrt{\mathcal{K}^{v_{i}} \frac{\left(N_{s}-v_{i}\right) !}{v_{i} ! N_{s} !}}\left(b_{i}^{\dagger}\right)^{v_{i}}|0\rangle .
$$

The matrix elements of the Hamiltonian (239) are obtained by means of (234) and

$$
\begin{gathered}
b_{i}^{\dagger}\left|\left[N_{s}\right] ; v_{i}\right\rangle=\sqrt{\left(v_{i}+1\right)\left(1-\frac{v_{i}+1}{\kappa}\right)}\left|\left[N_{s}\right] ; v_{i}+1\right\rangle, \\
b_{i}\left|\left[N_{s}\right] ; v_{i}\right\rangle=\sqrt{v_{i}\left(1-\frac{v_{i}}{\kappa}\right)}\left|\left[N_{s}\right] ; v_{i}-1\right\rangle .
\end{gathered}
$$

We proceed to apply this approach to obtain the PES of carbon dioxide in its electronic ground state. In this electronic manifold, $\mathrm{CO}_{2}$ is a linear molecule with a bond 
TABLE 9: Force constants for carbon dioxide from [68].

\begin{tabular}{lcc}
\hline Force constant & $\begin{array}{c}{ }^{12} \mathrm{CO}_{2} \\
\text { This work }\end{array}$ & $\begin{array}{c}{ }^{12} \mathrm{CO}_{2} \\
\text { Reference [136] }\end{array}$ \\
\hline$f_{q_{1} q_{1}}\left(\mathrm{aJ} \AA^{-2}\right)$ & 15.98 & 16.01 \\
$f_{q_{1} q_{2}}\left(\mathrm{aJ} \AA^{-2}\right)$ & 1.5310 & 1.2526 \\
$f_{q_{1} q_{1} q_{1} q_{1}}\left(\mathrm{aJ} \AA^{-4}\right)$ & 189.61 & 681.87 \\
$f_{q_{1} q_{1} q_{1} q_{2}}\left(\mathrm{aJ} \AA^{-4}\right)$ & 152.73 & 12.27 \\
$f_{q_{1} q_{1} q_{2} q_{2}}\left(\mathrm{aJ} \AA^{-4}\right)$ & 59.367 & 36.702 \\
$f_{q_{a} q_{a}}\left(\mathrm{a} J \AA^{-2}\right)$ & 0.5721 & 0.5818 \\
$f_{q_{a} q_{a} q_{a} q_{a}}\left(\mathrm{aJ} \AA^{-4}\right)$ & 3.3714 & 0.75 \\
& -7.1862 & - \\
$f_{q_{1} q_{a} q_{a}}\left(\mathrm{aJ} \AA^{-3}\right)$ & -0.9592 & -0.8874 \\
$f_{q_{1} q_{1} q_{a} q_{a}}\left(\mathrm{a} J \AA^{-4}\right)$ & -19.549 & - \\
$f_{q_{1} q_{2} q_{a} q_{a}}\left(\mathrm{aJ} \AA^{-4}\right)$ & -32.880 & - \\
\hline
\end{tabular}

length $r_{e}=1.16 \AA$ [134]. Thus, its point symmetry group is $\mathscr{D}_{\infty h}$, with four vibrational degrees of freedom: two stretching modes $\left(\Sigma_{g}^{+} \oplus \Sigma_{u}^{+}\right)$and a doubly degenerate bending mode $\left(\Pi_{u}^{ \pm}\right)$[8]. The Hamiltonian (239) has 11 spectroscopic parameters. However, as shown in Appendix $C$ of [68], we know that the parameters $\alpha_{1}^{b}$ and $\alpha_{2}^{b}$ depend on the same force constant $f_{++--}$. Thus, we obtain two different estimates for the $f_{++--}$force constant because in the fit we considered these parameters as independent. This can be justified since, in principle, in a more complete description, additional force constants of higher order should affect differently both values. The spectroscopic parameters are optimized with an iterative nonlinear least square method. The fit included all the experimental energies up to polyad $P=9$. The optimal values for the boson numbers $N_{s}$ and $N$ were found to be $N_{s}=160$ and $N=150$. In Table C.1 of [68] the 101 fitted energies are given, where an rms deviation of $0.53 \mathrm{~cm}^{-1}$ was obtained. The set of optimized spectroscopic parameters that produced the fit in Table C. 1 are presented in Table C. 2 of [68].

From the spectroscopic parameters we calculate the force constants that characterize the ground electronic state of carbon dioxide. The results are shown in Table 9. As mentioned above, since we are considering effective force constants, two values for the force constant $f_{q_{a} q_{a} q_{a} q_{a}}$ were obtained due to the ambiguity in its determination. The results show that the general trend of the force constants is satisfactorily reproduced. The quadratic and cubic force constants obtained by our approach are close to the values given by Chedin $[135,136]$, as can be noted. A remarkable result regarding the Fermi parameter is that a change in sign provides the same fit. However, the predicted force constants as well as the wave functions may be quite different. Hence, a fit with $\alpha_{1}^{s b}=36.005 \mathrm{~cm}^{-1}$ gives rise to the force constant $f_{q_{1} q_{a} q_{a}}=-5.4 \mathrm{aJ} \mathrm{A}^{-3}$, while $\alpha_{1}^{s b}=-36.005 \mathrm{~cm}^{-1}$ gives rise to the force constant $f_{q_{1} q_{a} q_{a}}=-0.9592 \mathrm{aJ} \mathrm{A}^{-3}$, close to the force constant $f_{q_{1} q_{a} q_{a}}=-0.8874$ aJ A ${ }^{-3}$ obtained in $[135,136]$. However, the accordance between our results and Chedin's force constants in the quartic order case is sensitively worse.
This is an expected result, considering the difference between both approaches.

This system is a representative case where a good fit does not guarantee good wave functions. The eigenvectors have to be tested through the calculation of Raman transition intensities, for instance [137].

\section{Conclusions}

In this paper we have presented a review of the recent developments in establishing the connection between algebraic methods based on unitary groups and the configuration space, the basic ingredient to extract the potential energy surfaces. To achieve this goal we present the vibrational description of representative molecules where the different algebraic methods as well as physical situations are present.

This contribution starts presenting a summary of the ro-vibrational description of molecules in the framework of space of the molecular coordinates. The vibrational Hamiltonian in terms of curvilinear coordinates is also discussed. Because of the importance of symmetry of the systems, the basic concepts involved in the symmetry projection technique based on the eigenvector method has been presented. A remarkable bonus of this approach is that the character tables are naturally identified with sets of quantum numbers. This approach has been presented in the framework of the the permutation inversion formalism, which is fundamental in establishing the molecular symmetry group.

The basic concepts involved in an algebraic approach is presented through the $S U(2)$ model, the most simple model associated with the vibrational degrees of freedom. Concepts of dynamical symmetry and chains of groups are illustrated. The connection of the $S U(2)$ algebraic model with the Morse potential is established in detail, providing the expansion of the coordinate and momentum in terms of the generators of the group. Once the analysis of one oscillator is established, we study the case of two interacting oscillators. This study provides a criterion to determine the local character of a molecule, but it also shows that the algebraic treatment of anharmonization does not have analog in configuration space when dealing with molecules with a normal mode behavior preserving the polyad. The extention of the model to a set of interacting Morse or/and PT oscillators permits to describe the vibrational excitations of polyatomic molecules. Hence, an approach for molecules with both local and normal mode behaviors has been established in a unified form in the framework of local interacting Morse oscillators.

One of the traditional approaches to extract the PES in the framework of the $U(v+1)$ algebraic models is based on coherent states. We have presented the suitable transformations to recover the PES for the case of two interacting Morse oscillators. Although this approach can be extended to more than two oscillators, the transformation is restricted to the case of interactions up to quadratic order. More work is needed in order to extend the treatment to Hamiltonians of any order.

Water was the first molecule to be analyzed as an example to obtain the PES through the connection of the unitary approach with the coordinates and momenta going beyond 
the linear approximation. Up to quadratic terms are taken into account in the expansion, which allows the full PES to be obtained up to quartic order even preserving the polyad. The more elaborated molecular system of trifluoride of boron was presented in order to show the power of the algebraic model when applied to molecules with normal mode behavior. This system was interesting not only because of its normal mode behavior but also because the approach followed to deal with spurious states.

Regarding the $U(v+1)$ model of $v$-equivalent oscillators, we have presented a reformulation that allows the force constants to be obtained. In this scheme the difference between this approach and the models of interacting Morse oscillators is clearly established. This approach is applied to the pyramidal molecule Arsine. It has been also shown how to model the dipole transition probabilities.

The equivalent approach to extract the PES is presented for the case of linear triatomic molecules. As an example the carbon dioxide has been analyzed. This approach is general for any linear molecule as long as the polyad is considered as a good quantum number.

Summarizing, we have presented a general approach to estimate the PES when algebraic methods based on unitary algebras are used. This is based on the connection between the algebraic approach and the configuration space. Only in the most simple case of the $S U(2)$ model the connection can be established in exact form. For higher dimension only an approximation can be proposed. This approximation, however, is enough to obtain a reasonable PES for the case of semirigid molecules. We believe that these efforts to connect the algebraic approaches with configuration space may represent an important step for estimating the PES in a simple way. The algebraic approach contrasts with variational methods, which are too expensive to be applied to large- or even medium- sized molecules.

\section{Acknowledgment}

This work is partially supported by CONACyT, Mexico.

\section{References}

[1] M. Born and K. Huang, Dynamical Theory of Crystal Lattices, Clarendon Press, Oxford, UK, 1954.

[2] P. R. Bunker and P. Jensen, "The born-oppenheimer approximation," in Computational Molecular Spectroscopy, P. Jensen and P. R. Bunker, Eds., John Wiley \& Sons, New York, NY, USA, 2000.

[3] P. R. Bunker and P. Jensen, Molecular Symmetry and Spectroscopy, National Research Council of Canada, 1998.

[4] D. Papoušek and M. R. Aliev, Molecular VibrationalRotational Spectra, Elsevier, Amsterdam, The Netherlands, 1982.

[5] W. West, Ed., Chemical Applications of Spectroscopy. Microwave Molecular Spectra, Interscience, New York, NY, USA, 1970.

[6] P. Pyykkö, "Relativistic effects in structural chemistry," Chemical Reviews, vol. 88, no. 3, pp. 563-594, 1988.

[7] S. N. Yurchenko, M. Carvajal, P. Jensen, F. Herregodts, and T. R. Huet, "Potential parameters of $\mathrm{PH}_{3}$ obtained by simultaneous fitting of ab initio data and experimental vibrational band origins," Chemical Physics, vol. 290, no. 1, pp. 59-67, 2003.

[8] G. G. Herzberg, Molecular Spectra and Molecular Structure. II. Infrared and Raman Spectra of Polyatomic Molecules, Krieger Publishing Company, Malabar, Fla, USA, 1991.

[9] S. Califano, Vibrational States, John Wiley \& Sons, New York, NY, USA, 1976.

[10] R. T. Lawton and M. S. Child, "Excited stretching vibrations of water: the quantum mechanical picture," Molecular Physics, vol. 40, no. 4, pp. 773-792, 1940.

[11] M. S. Child and L. Halonen, "Overtone frequencies and intensities in the local mode picture," Advances in Chemical Physics, vol. 57, pp. 1-57, 1984.

[12] L. Halonen, "Local mode vibrations in polyatomic molecules," Advances in Chemical Physics, vol. 104, pp. 41-179, 1998.

[13] P. Jensen, "An introduction to the theory of local mode vibrations," Molecular Physics, vol. 98, no. 17, pp. 1253-1285, 2000.

[14] M. Moshinsky and Y. F. Smirnov, The Harmonic Oscillator in Modern Physics, Harwood Academic Publishers, NewYork, NY, USA, 1996.

[15] M. E. Kellman, "Approximate constants of motion for vibrational spectra of many-oscillator systems with multiple enharmonic resonances," The Journal of Chemical Physics, vol. 93, no. 9, pp. 6630-6635, 1990.

[16] M. Moshinsky, Group Theory and the Many Body Problem, Gordon and Breach, New York, NY, USA, 1967.

[17] J. Hinze, Ed., The Unitary Group for the Evaluation of Electronic Energy Matrix Elements, Unitary Group Workshop, 1979.

[18] F. A. Matsen and R. Pauncz, The Unitary Group in Quantum Chemistry, Studies in Physical and Theoretical Chemistry, Elsevier, 1986.

[19] A. Frank and P. Van Isacker, Algebraic Methods in Molecular and Nuclear Structure Physics, John Wiley \& Sons, New York, NY, USA, 1994.

[20] R. Lemus and A. Frank, "Approximate dynamical symmetry in the first series of hydride diatomic molecules," Physical Review Letters, vol. 66, no. 22, pp. 2863-2866, 1991.

[21] I. Talmi, Simple Models of Complex Nuclei. The Shell Model and the Interacting Boson Model, Harwood Academic Publishers, NewYork, NY, USA, 1993.

[22] F. 1. Stancu, Group Theory in Subnuclear Physics, Oxford Science Publications, 1996.

[23] F. Iachello and R. D. Levine, Algebraic Theory of Molecules, Oxford University Press, New York, NY, USA, 1995.

[24] S. Oss, "Algebraic models in molecular spectroscopy," Advances in Chemical Physics, vol. 93, pp. 455-649, 1996.

[25] H. J. Lipkin, Lie Groups for Pedestrians, Dover, New York, NY, USA, 2002.

[26] B. G. Wybourne, Classical Groups for Physicists, John Wiley \& Sons, New York, NY, USA, 1974.

[27] D. Janssen, R. V. Jolos, and F. Dönau, "An algebraic treatment of the nuclear quadrupole degree of freedom," Nuclear Physics, Section A, vol. 224, no. 1, pp. 93-115, 1974.

[28] F. Iachello and A. Arima, The Interacting Boson Model, Cambridge University Press, Cambridge, UK, 1987.

[29] F. Iachello, "Algebraic methods for molecular rotation-vibration spectra," Chemical Physics Letters, vol. 78, no. 3, pp. 581$585,1981$.

[30] F. Iachello and R. D. Levine, "Algebraic approach to molecular rotation-vibration spectra. I. Diatomic molecules," The 
Journal of Chemical Physics, vol. 77, no. 6, pp. 3046-3055, 1982.

[31] F. Iachello, S. Oss, and R. Lemus, "Vibrational spectra of linear triatomic molecules in the vibron model," Journal of Molecular Spectroscopy, vol. 146, no. 1, pp. 56-78, 1991.

[32] F. Iachello, S. Oss, and R. Lemus, "Linear four-atomic molecules in the vibron model," Journal of Molecular Spectroscopy, vol. 149, no. 1, pp. 132-151, 1991.

[33] R. Bijker and A. Leviatan, "Algebraic treatment of three-body problems," Few-Body Systems, vol. 25, no. 1-3, pp. 89-100, 1998.

[34] F. Iachello, F. Pérez-Bernal, and P. H. Vaccaro, "A novel algebraic scheme for describing nonrigid molecules," Chemical Physics Letters, vol. 375, no. 3-4, pp. 309-320, 2003.

[35] F. Pérez-Bernal, L. F. Santos, P. H. Vaccaro, and F. Iachello, "Spectroscopic signatures of nonrigidity: algebraic analyses of infrared and Raman transitions in nonrigid species," Chemical Physics Letters, vol. 414, no. 4-6, pp. 398-404, 2005.

[36] F. Pérez-Bernal and F. Iachello, "Algebraic approach to twodimensional systems: shape phase transitions, monodromy, and thermodynamic quantities," Physical Review A, vol. 77, no. 3, 21 pages, 2008.

[37] F. Iachello and F. Prez-Bernal, "Bending vibrational modes of ABBA molecules: algebraic approach and its classical limit," Molecular Physics, vol. 106, no. 2-4, pp. 223-231, 2008.

[38] F. Iachello and F. Pérez-Bernal, "A novel algebraic scheme for describing coupled benders in tetratomic molecules," Journal of Physical Chemistry A, vol. 113, no. 47, pp. 13273-13286, 2009.

[39] A. Frank, F. Iachello, and R. Lemus, "Algebraic methods for molecular electronic spectra," Chemical Physics Letters, vol. 131, no. 4-5, pp. 380-383, 1986.

[40] A. Frank, R. Lemus, and F. Iachello, "Algebraic approach to molecular electronic spectra. I. Energy levels," The Journal of Chemical Physics, vol. 91, no. 1, pp. 29-41, 1989.

[41] R. Lemus and A. Frank, "An algebraic model for molecular electronic excitations in diatomic molecules," Annals of Physics, vol. 206, no. 1, pp. 122-192, 1991.

[42] R. Lemus, A. Leviatan, and A. Frank, "Potential energy curves in the electron-vibron model," Chemical Physics Letters, vol. 194, no. 4-6, pp. 327-332, 1992.

[43] R. Lemus and A. Frank, "Constrained calculations in the electron-vibron model and the Born-Oppenheimer approximation," Physical Review A, vol. 47, no. 6, pp. 4920-4933, 1993.

[44] F. Michelot and J. Moret-bailly, "Algebraic approach to vibrational spectra of polyatomic molecules Approche algébrique des spectres vibrationnels des molécules polyatomiques," Journal of Physics, vol. 48, no. 1, pp. 51-72, 1987.

[45] C. Leroy and F. Michelot, "The local group $\mathrm{K}(4)$ in the algebraic approach to vibrational spectra of tetrahedral molecules: application to silane," Journal of Molecular Spectroscopy, vol. 151, no. 1, pp. 71-96, 1992.

[46] C. Leroy, F. Collin, and M. Loëte, "Algebraic approach to vibrational spectra of tetrahedral molecules: first order infrared intensity model," Journal of Molecular Spectroscopy, vol. 175, no. 2, pp. 289-295, 1996.

[47] L. Pluchart, C. Leroy, and A. Mourbat, "Study of the stretching modes of the arsine molecule," Journal of Molecular Spectroscopy, vol. 218, no. 1, pp. 1-11, 2003.

[48] L. Pluchart, C. Leroy, N. Sanzharov, F. Michelot, E. Bekhtereva, and O. Ulenikov, "Vibrational modes of the stibine molecule," Journal of Molecular Spectroscopy, vol. 232, no. 2, pp. 119-136, 2005.
[49] J. M. Arias, A. Frank, R. Lemus, and F. Pérez-Bernal, "Algebraic description of stretching and bending modes in non-linear triatomic molecules," Revista Mexicana de Física S, vol. 41, no. 5, pp. 703-727, 1995.

[50] F. Iachello and S. Oss, "Algebraic model of bending vibrations of complex molecules," Chemical Physics Letters, vol. 205, no. 2-3, pp. 285-289, 1993.

[51] O. S. van Roosmalen, Algebraic descriptions of nuclear and molecular rotationvibration spectra, Ph.D. thesis, 1982.

[52] O. S. van Roosmalen, R. D. Levine, and A. E. L. Dieperink, "The geometrical-classical limit of algebraic Hamiltonians for molecular vibrotational spectra," Chemical Physics Letters, vol. 101, no. 6, pp. 512-517, 1983.

[53] O. Castaños and R. Lemus, "Potential energy surfaces in algebraic molecular models using coherent states," Molecular Physics, vol. 108, no. 5, pp. 597-610, 2010.

[54] A. Frank, R. Lemus, M. Carvajal, C. Jung, and E. Ziemniak, "SU(2) approximation to the coupling of Morse oscillators," Chemical Physics Letters, vol. 308, no. 1-2, pp. 91-98, 1999.

[55] M. Carvajal, R. Lemus, A. Frank, C. Jung, and E. Ziemniak, "An extended SU(2) model for coupled Morse oscillators," Chemical Physics, vol. 260, no. 1-2, pp. 105-123, 2000.

[56] R. Lemus and R. Bernal, "Connection of the vibron model with the modified Pöschl-Teller potential in configuration space," Chemical Physics, vol. 283, no. 3, pp. 401-417, 2002.

[57] R. Lemus, M. Carvajal, J. C. López-V., and A. Frank, "Spectroscopic description of $\mathrm{H}_{2} \mathrm{O}$ in the $\mathrm{su}(2)$ vibron model approximation," Journal of Molecular Spectroscopy, vol. 214, no. 1, pp. 52-68, 2002.

[58] R. Lemus, "Vibrational excitations in $\mathrm{H}_{2} \mathrm{O}$ in the framework of a local model," Journal of Molecular Spectroscopy, vol. 225, no. 1, pp. 73-92, 2004.

[59] M. Sánchez-Castellanos, C. A. Amezcua-Eccius, O. ÁlvarezBajo, and R. Lemus, "A local-normal description of vibrational excitations of pyramidal molecules in terms of Morse oscillators," Journal of Molecular Spectroscopy, vol. 247, no. 2, pp. 140-159, 2008.

[60] R. Bernal and R. Lemus, "Algebraic local vibrational spectroscopic description of formaldehyde," Journal of Molecular Spectroscopy, vol. 235, no. 2, pp. 218-234, 2006.

[61] M. Sánchez-Castellanos and R. Lemus, "Force field constants for Formaldehyde obtained from an algebraic approach," Journal of Molecular Spectroscopy, vol. 266, no. 1, pp. 1-11, 2011.

[62] C. A. Amezcua-Eccius and R. Lemus, "An approach for the description of vibrational excitations: application to ${ }^{11} \mathrm{BF}_{3}$," Journal of Molecular Spectroscopy, vol. 260, no. 1, pp. 36-49, 2010.

[63] O. Álvarez-Bajo, M. Sánchez-Castellanos, C. A. AmezcuaEccius, and R. Lemus, "Force constants and transition intensities in the $U(v+1)$ model for molecular vibrational excitations," Journal of Molecular Spectroscopy, vol. 237, no. 2, pp. 247-258, 2006.

[64] O. Álvarez-Bajo, M. Sánchez-Castellanos, and R. Lemus, "Some considerations on the description of vibrational excitations in terms of $\mathrm{U}(\nu+1)$ unitary groups," Journal of Molecular Spectroscopy, vol. 236, no. 1, pp. 134-146, 2006.

[65] M. Sánchez-Castellanos, O. Álvarez-Bajo, C. A. AmezcuaEccius, and R. Lemus, "Vibrational excitations of arsine in the framework of a local unitary group approach," Journal of Molecular Spectroscopy, vol. 240, no. 1, pp. 81-92, 2006.

[66] C. A. Amezcua-Eccius, O. Álvarez-Bajo, M. SánchezCastellanos, and R. Lemus, "A local description of vibrational excitations of stibine in the framework of a unitary group 
approach," Journal of Molecular Spectroscopy, vol. 240, no. 2, pp. 164-173, 2006.

[67] F. Iachello, "Algebraic approach to molecular spectra: twodimensional problems," The Journal of Chemical Physics, vol. 104, no. 18, pp. 6956-6963, 1996.

[68] M. Sánchez-Castellanos, R. Lemus, M. Carvajal, and F. PérezBernal, "A novel connection between algebraic spectroscopic parameters and force constants in the description of vibrational excitations of linear triatomic molecules," Journal of Molecular Spectroscopy, vol. 253, no. 1, pp. 1-15, 2009.

[69] R. Lemus and M. Sánchez-Castellanos, "A study of vibrational excitations of $\mathrm{HCN}$ in the framework of an algebraic model," Molecular Physics, vol. 109, no. 16, pp. 2009-2023, 2011.

[70] S. N. Yurchenko, M. Carvajal, H. Lin, J. Zheng, W. Thiel, and P. Jensen, "Dipole moment and rovibrational intensities in the electronic ground state of $\mathrm{NH}_{3}$ : bridging the gap between ab initio theory and spectroscopic experiment," The Journal of Chemical Physics, vol. 122, no. 10, Article ID 104317, 14 pages, 2005.

[71] F. L. Pilar, Elementary Quantum Chemistry, McGraw-Hill, New York, NY, USA, 1968.

[72] A. Szabo and N. S. Ostlund, Modern Quantum Chemistry, McGraw-Hill, New York, NY, USA, 1982.

[73] M. E. Rose, Elementary Theory of Angular Momentum, Dover, New York, NY, USA, 1957.

[74] C. Eckart, "Some studies concerning rotating axes and polyatomic molecules," Physical Review, vol. 47, no. 7, pp. 552-558, 1935.

[75] E. B. Wilson, J. C. Decius, and P. C. Cross, Molecular Vibrations, McGraw-Hill, New York, NY, USA, 1995.

[76] K. G. Watson, "Simplification of the molecular vibrationrotation hamiltonian," Molecular Physics, vol. 15, no. 5, pp. 479-490, 1968.

[77] J. D. Louck, "Derivation of the molecular vibration-rotation Hamiltonian from the Schrödinger equation for the molecular model," Journal of Molecular Spectroscopy, vol. 61, no. 1, pp. 107-137, 1976.

[78] J. M. Hollas, High Resolution Spectroscopy, John Wiley \& Sons, New York, NY, USA, 2nd edition, 1992.

[79] M. Quack and F. Merkt, Eds., Handbook of High-Resolution Spectroscopy, John Wiley \& Sons, New York, NY, USA, 2nd edition, 2011.

[80] H. M. Pickett, "Vibration-rotation interactions and the choice of rotating axes for polyatomic molecules," The Journal of Chemical Physics, vol. 56, no. 4, pp. 1715-1723, 1972.

[81] R. Meyer and H. H. Günthard, "General internal motion of molecules, classical and quantum-mechanical hamiltonian," The Journal of Chemical Physics, vol. 49, no. 4, pp. 1510-1520, 1968.

[82] P. Jensen, "A new morse oscillator-rigid bender internal dynamics (MORBID) Hamiltonian for triatomic molecules," Journal of Molecular Spectroscopy, vol. 128, no. 2, pp. 478501, 1988.

[83] E. P. Wigner, Group Theory and Its Applicactions to the Quantum Theory of Atomic Spectra, Academic Press, New York, NY, USA, 1959.

[84] H. C. Longuet-Higgins, "The symmetry groups of non-rigid molecules," Molecular Physics, vol. 6, no. 5, pp. 445-460, 1963.

[85] J. T. Hougen, "Classification of rotational energy levels for symmetric-top molecules," The Journal of Chemical Physics, vol. 37, no. 6, pp. 1433-1441, 1962.
[86] J. T. Hougen, "Classification of rotational energy levels. II," The Journal of Chemical Physics, vol. 39, no. 2, pp. 358-365, 1963.

[87] C. Cohen-Tannoudji, B. Diu, and F. Laloë, Quantum Mechanics, vol. 1, John Wiley \& Sons, New York, NY, USA, 1977.

[88] J. Q. Chen, Group Representation Theory for Physicists, World Scientific, Singapore, 1989.

[89] R. Lemus, "A general method to obtain vibrational symmetry adapted bases in a local scheme," Molecular Physics, vol. 101, no. 16, pp. 2511-2528, 2003.

[90] O. Álvarez-Bajo, R. Lemus, M. Carvajal, and F. PérezBernal, "Equivalent rotations associated with the permutation inversion group revisited: symmetry projection of the rovibrational functions of methane," Molecular Physics, vol. 109, no. 5, pp. 797-812, 2011.

[91] M. E. Kellman, "Algebraic methods in spectroscopy," Annual Review of Physical Chemistry, vol. 46, pp. 354-421, 1995.

[92] R. Gilmore, Lie Groups, Lie Algebras, and Some of Their Applications, Dover, New York, NY, USA, 2002.

[93] P. M. Morse, "Diatomic molecules according to the wave mechanics. II. Vibrational levels," Physical Review, vol. 34, no. 1, pp. 57-64, 1929.

[94] S.-H. Dong, R. Lemus, and A. Frank, "Ladder operators for the Morse potential," International Journal of Quantum Chemistry, vol. 86, no. 5, pp. 433-439, 2002.

[95] V. Aldaya and J. Guerrero, "Group approach to the quantization of the Pöschl-Teller dynamics," Journal of Physics A, vol. 38, no. 31, pp. 6939-6953, 2005.

[96] J. M. Arias, J. Gómez-Camacho, and R. Lemus, "An su(1, 1) dynamical algebra for the Pöschl-Teller potential," Journal of Physics A, vol. 37, no. 3, pp. 877-893, 2004.

[97] R. Lemus, J. M. Arias, and J. Gómez-Camacho, “An su(1, 1) dynamical algebra for the Morse potential," Journal of Physics A, vol. 37, no. 5, pp. 1805-1820, 2004.

[98] O. Alvarez-Bajo, J. M. Arias, J. Gomez-Camacho, and R. Lemus, "An approach to the study of the continuum effects in systems of interacting Morse oscillators," Molecular Physics, vol. 106, no. 9-10, pp. 1275-1289, 2008.

[99] J. Gómez-Camacho, R. Lemus, and J. M. Arias, "Matrix elements of $\mathrm{u}$ and $\mathrm{p}$ for the modified Pöschl-Teller potential," Journal of Physics A, vol. 37, no. 19, pp. 5237-5242, 2004.

[100] R. Lemus and A. Frank, "Systematic polyad mixing in a local mode model," Chemical Physics Letters, vol. 349, no. 5-6, pp. 471-476, 2001.

[101] M. Sánchez-Castellanos and R. Lemus, "A systematic polyad breaking approach to anharmonic systems," Journal of Physics $B$, vol. 41, no. 17, Article ID 175101, 2008.

[102] A. Leviatan and M. W. Kirson, "Intrinsic and collective structure of an algebraic model of molecular rotationvibration spectra," Annals of Physics, vol. 188, no. 1, pp. 142$185,1988$.

[103] O. S. van Roosmalen, I. Benjamin, and R. D. Levine, "A unified algebraic model description for interacting vibrational modes in ABA molecules," The Journal of Chemical Physics, vol. 81, no. 12, pp. 5986-5997, 1984.

[104] I. Benjamin and R. D. Levine, "Potential energy surfaces for stable triatomic molecules using an algebraic Hamiltonian," Chemical Physics Letters, vol. 117, no. 4, pp. 314-320, 1985.

[105] Y. Zheng and S. Ding, "Potential energy surface for linear triatomic molecules: an algebraic method," Journal of Mathematical Chemistry, vol. 28, no. 1-3, pp. 193-200, 2000. 
[106] E. L. Sibert III, "Theoretical studies of vibrationally excited polyatomic molecules using canonical Van Vleck perturbation theory," The Journal of Chemical Physics, vol. 88, no. 7, Article ID 4378, 13 pages, 1988.

[107] E. L. Sibert III, "VANVLK: an algebraic manipulation program for canonical Van Vleck perturbation theory," Computer Physics Communications, vol. 51, no. 1-2, pp. 149$160,1988$.

[108] H. G. Kjaergaard, B. R. Henry, H. Wei et al., "Calculation of vibrational fundamental and overtone band intensities of $\mathrm{H}_{2} \mathrm{O}$," The Journal of Chemical Physics, vol. 100, no. 9, pp. 6228-6239, 1994.

[109] L. Halonen and T. Carrington Jr., "Fermi resonances and local modes in water, hydrogen sulfide, and hydrogen selenide," The Journal of Chemical Physics, vol. 88, pp. 41714185, 1988.

[110] M. L. Sage and J. A. Williams III, "Energetics, wave functions, and spectroscopy of coupled anharmonic oscillators," The Journal of Chemical Physics, vol. 78, no. 3, pp. 1348-1358, 1983.

[111] M. L. Sage, "Morse oscillator transition probabilities for molecular bond modes," Chemical Physics, vol. 35, no. 3, pp. 375-380, 1978.

[112] L. Halonen and M. S. Child, "A local mode model for tetrahedral molecules," Molecular Physics, vol. 46, no. 2, pp. 239-255, 1982.

[113] L. Halonen, M. S. Child, and S. Carter, "Potential models and local mode vibrational eigenvalue calculations for acetylene," Molecular Physics, vol. 47, no. 5, pp. 1097-1112, 1982.

[114] L. Halonen, "Stretching vibrational overtone and combination states in silicon tetrafluoride," Journal of Molecular Spectroscopy, vol. 120, no. 1, pp. 175-184, 1986.

[115] B. T. Darling and D. M. Dennison, "The water vapor molecule," Physical Review, vol. 57, no. 2, pp. 128-139, 1940.

[116] A. Maki, T. Masiello, and T. A. Blake, "The high-resolution infrared spectrum of ${ }^{10} \mathrm{BF}_{3}$ from 400 to $4600 \mathrm{~cm}^{-1}$," Journal of Molecular Structure, vol. 742, no. 1-3, pp. 3-20, 2005.

[117] T. Masiello, A. Maki, and T. A. Blake, "The high-resolution infrared spectrum of ${ }^{11} \mathrm{BF}_{3}$ from 400 to $1650 \mathrm{~cm}^{-1}$," Journal of Molecular Spectroscopy, vol. 234, no. 1, pp. 122-136, 2005.

[118] A. Maki, J. K.G. Watson, T. Masiello, and T. A. Blake, "Observation of a forbidden $\mathrm{E}^{\prime \prime} \mathrm{s}(-) \mathrm{A} 1$ ' infrared transition in 11BF3," Journal of Molecular Spectroscopy, vol. 238, no. 2, pp. 135-144, 2006.

[119] R. Kirkpatrick, T. Masiello, A. Weber, and J. W. Nibler, "Coherent Raman spectra of the $v 1$ mode of ${ }^{10} \mathrm{BF}_{3}$ and ${ }^{11} \mathrm{BF}_{3}$," Journal of Molecular Spectroscopy, vol. 237, no. 1, pp. 97-103, 2006.

[120] T. Masiello, A. Maki, and T. A. Blake, "The analysis of combination and overtone states of ${ }^{11} \mathrm{BF}_{3}$ from 1650 to 4600 $\mathrm{cm}^{-1}$," Journal of Molecular Spectroscopy, vol. 243, no. 1, pp. 16-31, 2007.

[121] A. Frank, R. Lemus, F. Pérez-Bernal, and R. Bijker, "On the elimination of spurious modes in algebraic models of molecular vibrations," Journal of Molecular Spectroscopy, vol. 196, no. 2, pp. 329-334, 1999.

[122] T. Lukka, E. Kauppi, and L. Halonen, "Fermi resonances and local modes in pyramidal $\mathrm{XH}_{3}$ molecules: an application to arsine $\left(\mathrm{AsH}_{3}\right)$ overtone spectra," The Journal of Chemical Physics, vol. 102, no. 13, Article ID 5200, 7 pages, 1995.

[123] P. W. Atkins, Molecular Quantum Mechanics, Oxford University Press, Oxford, UK, 2nd edition, 1983.
[124] M. Halonen, L. Halonen, H. Bürger, and P. Moritz, "Stretching vibrational overtone spectrum of arsine and stibine," Journal of Physical Chemistry, vol. 96, no. 11, pp. 4225-4231, 1992.

[125] H. Lin, O. N. Ulenikov, S. Yurchinko, X-G. Wang, and Q.S. Zhu, "High-resolution spectroscopic study of the local mode (310) combinaiton band systems of $\mathrm{AsH}_{3}$," Journal of Molecular Spectroscopy, vol. 187, pp. 89-96, 1998.

[126] J. Breidung and W. Thiel, "The anharmonic force fields of arsine, stibine, and bismutine," Journal of Molecular Spectroscopy, vol. 169, no. 1, pp. 166-180, 1995.

[127] R. Mecke, Z. Elektrochem, vol. 54, pp. 38-51, 1950.

[128] F. M. Fernández and E. A. Castro, Algebraic Methods in Quantum Chemistry and Physics, CRC Press, New York, NY, USA, 1952.

[129] A. R. Hoy, I. M. Mills, and G. Strey, "Anharmonic force constant calculations," Molecular Physics, vol. 24, no. 6, pp. 1265-1290, 1972.

[130] M. Hamermesh, Group Theory and Its Applications to Physical Problems, Addison-Wesley, Reading, Mass, USA, 1962.

[131] T. Sako, D. Aoki, K. Yamanouchi, and F. Iachello, "Algebraic force-field Hamiltonian expansion approach to linear polyatomic molecules," The Journal of Chemical Physics, vol. 113, no. 15, pp. 6063-6069, 2000.

[132] H. Ishikawa, H. Toyosaki, N. Mikami, F. Pérez-Bernal, P. H. Vaccaro, and F. Lachello, "Algebraic analysis of bentfrom-linear transition intensities: the vibronically resolved emission spectrum of methinophosphide (HCP)," Chemical Physics Letters, vol. 365, no. 1-2, pp. 57-68, 2002.

[133] A. Frank, R. Lemus, R. Bijker, F. Pérez-Bernal, and J. M. Arias, "A general algebraic model for molecular vibrational spectroscopy," Annals of Physics, vol. 252, no. 1, pp. 211-238, 1996.

[134] G. Maroulis, "Electric (hyper) polarizability derivatives for the symmetric stretching of carbon dioxide," Chemical Physics, vol. 291, no. 1, pp. 81-95, 2003.

[135] A. Chedin, "The carbon dioxide molecule. Potential, spectroscopic, and molecular constants from its infrared spectrum," Journal of Molecular Spectroscopy, vol. 76, no. 1-3, pp. 430491, 1979.

[136] A. Chedin and J.-L. Teffo, "The carbon dioxide molecule: a new derivation of the potential, spectroscopic, and molecular constants," Journal of Molecular Spectroscopy, vol. 107, no. 2, pp. 333-342, 1984.

[137] M. Sánchez-Castellanos, M. Carvajal, and R. Lemus, "Description of the Raman sprectrum of $\mathrm{CO}_{2}$ using an algebraic approach," Chemical Physics Letters. In press.

[138] P. Jensen, "The potential energy surface for the electronic ground state of the water molecule determined from experimental data using a variational approach," Journal of Molecular Spectroscopy, vol. 133, no. 2, pp. 438-460, 1989.

[139] R. J. Bartlett, S. J. Cole, G. D. Purvis, W. C. Ermler, H. C. Hsieh, and I. Shavitt, "The quartic force field of $\mathrm{H}_{2} \mathrm{O}$ determined by many-body methods. II. Effects of triple excitations," The Journal of Chemical Physics, vol. 87, no. 11, pp. 6579-6591, 1987.

[140] Y. Pak and R. C. Woods, "Anharmonic force fields and spectroscopic properties of $\mathrm{BF}_{3}$ and $\mathrm{CF}_{3}^{+}$using the coupled cluster method," The Journal of Chemical Physics, vol. 106, no. 15, pp. 6424-6429, 1997. 


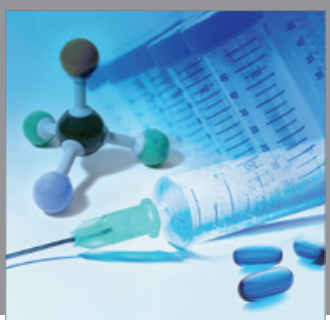

International Journal of

Medicinal Chemistry

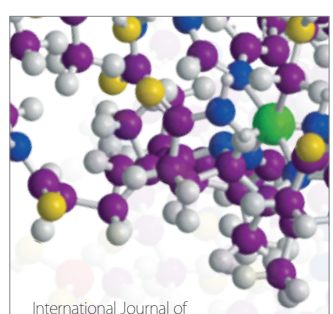

Carbohydrate Chemistry

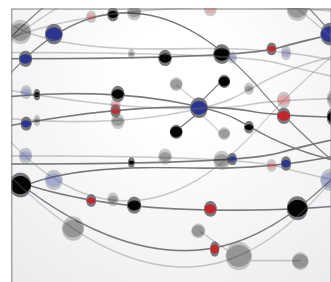

The Scientific World Journal
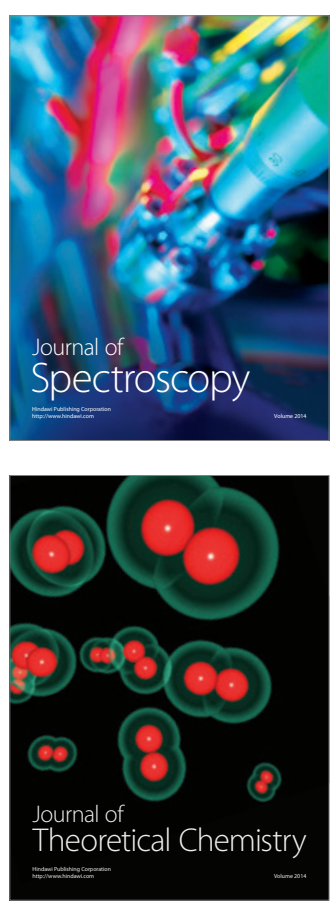
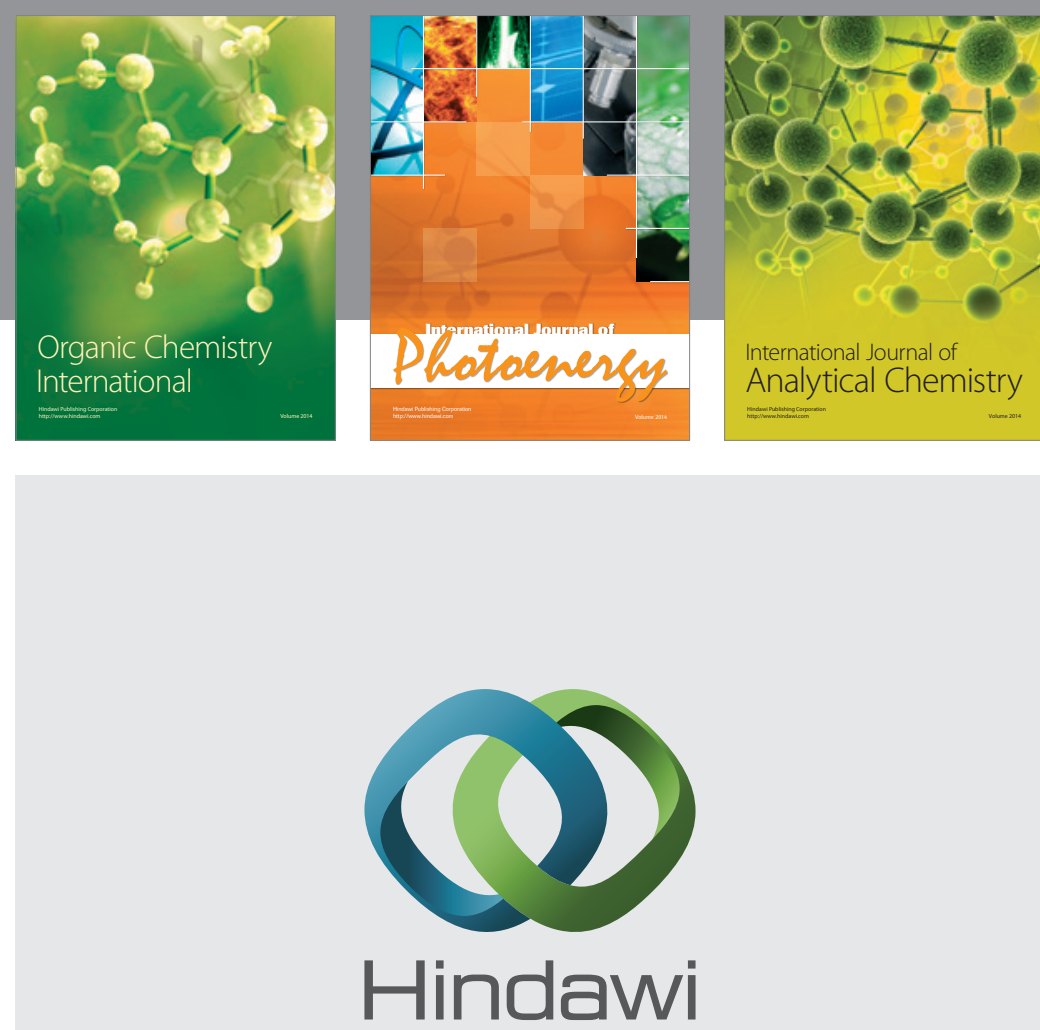

Submit your manuscripts at

http://www.hindawi.com
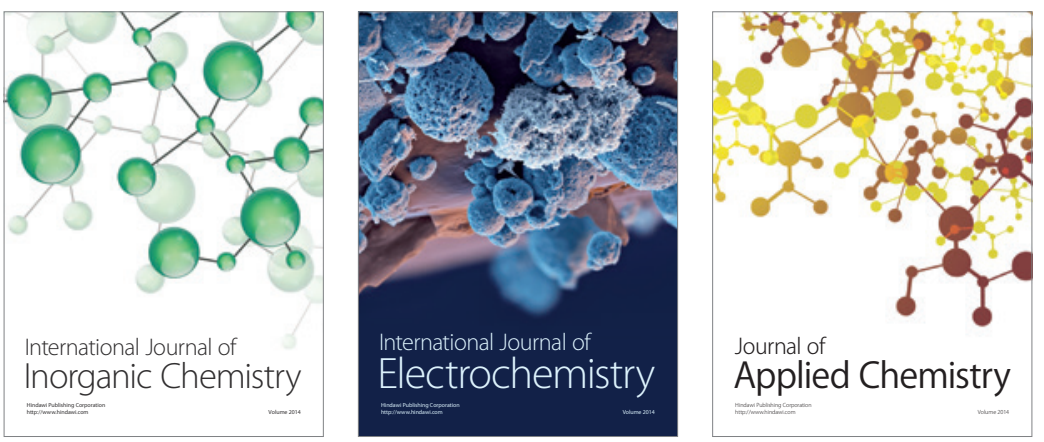

Journal of

Applied Chemistry
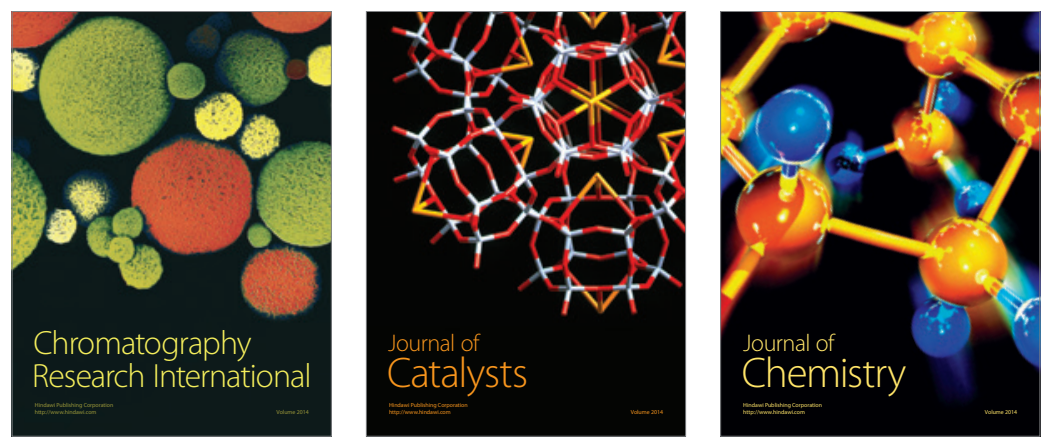
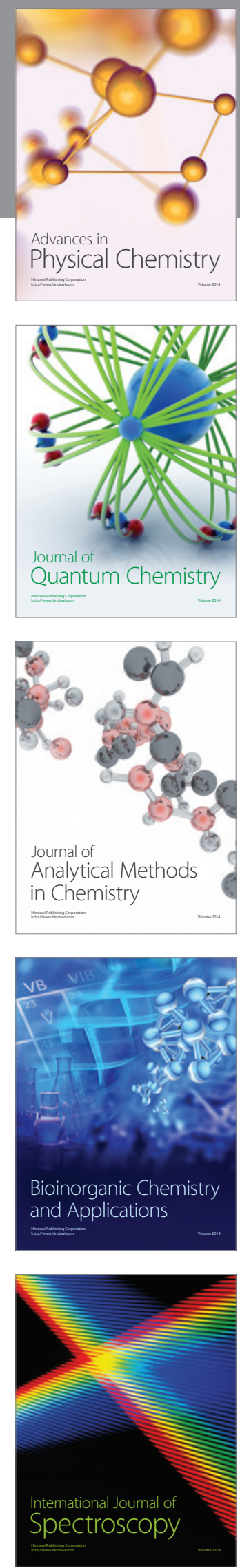